Eprisconal shunch botechisio

\title{
PM
}

1024

E69

1896

SOA

\section{OKODAKIGIYE WAKAN}

\section{WIWIGAWANGAPI \\ ITEOWAPI YUKAN}

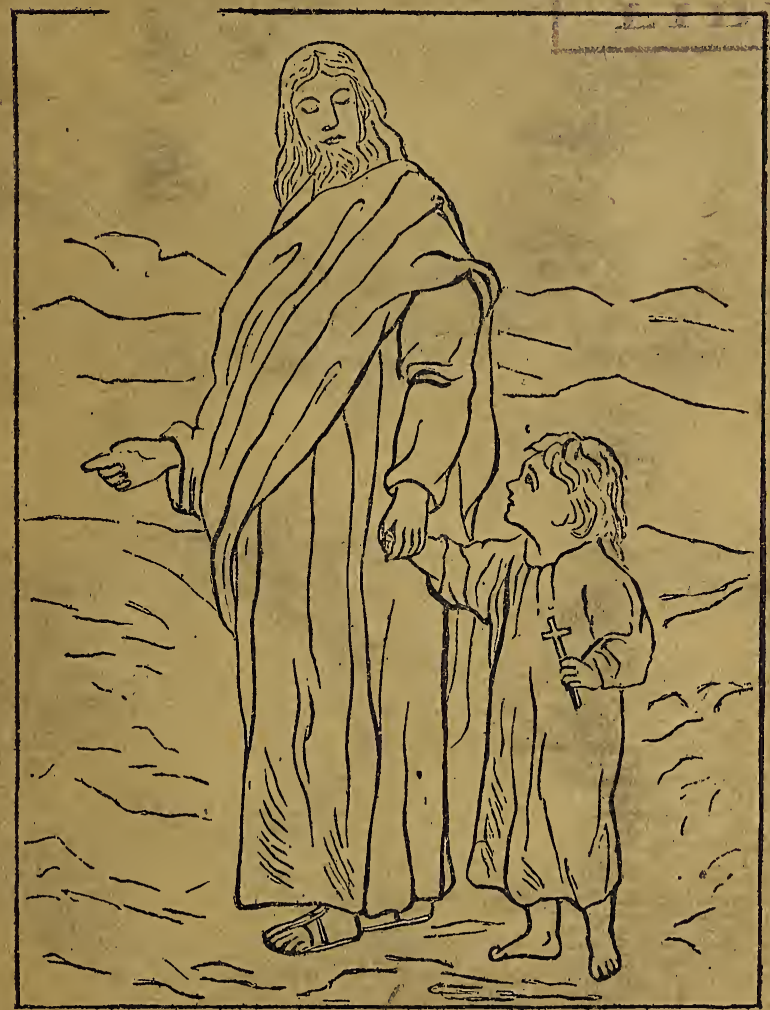

MRS. C. H. SMITH, OWA.

REV. WM: J. CLEVELAND, IESKA KAGA.

"Wiyacinpi cona takuna ewicakiye xni."

St. Matt. xiii, 34 .

MADISON, SOUTH DAKOTA.

DAILY LEADER PRINT.

1896.

\section{$f$}

PM1024

p.8s

1896

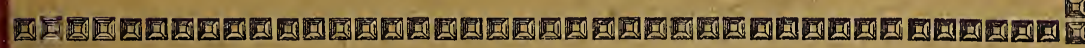




\section{THE}

\section{CHURCH Catechism}

SIMPLIFIED BY

\section{SHORT QUESTIONS AND ANSWERS}

Together witit Ili.ustrations

INTENDED FOR THE YOUNGER CHLLDREN OF THE CHURCE

BY MRS. C. H. SMITH 


\title{
OKODAKIGIYE WAKAN
}

\author{
WIWIGAWANGAPI
}

ITEOWAPI YUKAN, QA

WOIWANGE QA WOAYIPTE PTEPTEGENA

ON

TERIKE XNI KAGAPI.

OKODAKICIYE WAKAN KIN TAWAKANHEJA ONSPEWICAKIYAPI KTA
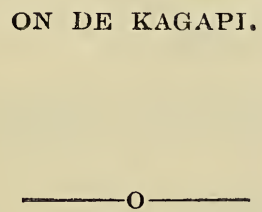

BUREAU OF

AMERICAN ETHANOLOGY

11.40

1807

II I IR A IR W

MRS. C. H. SMITH, OWA.

REV. WM. J. CLEVELAND, IEsKa Kaga. 


$$
\begin{aligned}
& \text { pM/ozh } \\
& .855 \\
& 1896 \\
& +193
\end{aligned}
$$


roe thok

811

$\$$

- Vegolondta

3.0 , sotgatash

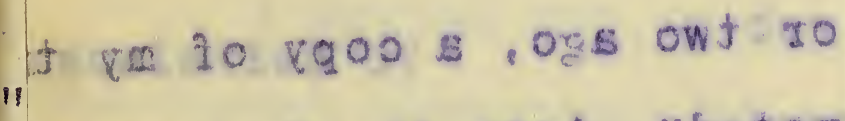
b motans ent ofnl "betsis 10

. bris Leve L. b co Dns ent benoltas grods 200

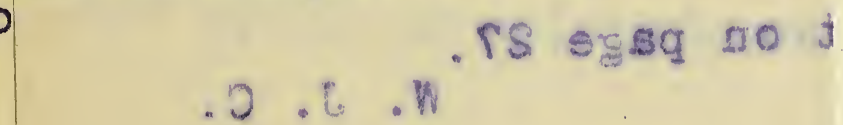



Madison, S. D.,

$1 / 9 / 1802$.

$\$$

Director of the bureau of Ethnology,

Washington, D. C.,

Sir :

I mailed you, a day or two ago, a copy of my translation of "The Church Catechism Illustrated", into the Yaniston dialect of the Dasota.

Very truly yours,

W. J. Cleveland.

P. S. Please insert in the boos above nentioned the enclosed slip, to correct error in printing cut on page 27 . W. J. C. 
to wserud ent to rotagito

$$
\text { : xईz }
$$

VBb s woV belifex I

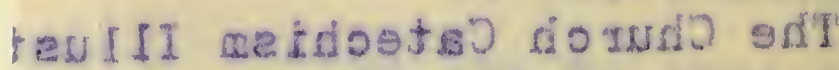

$$
.6 \%
$$

$$
\text { - exuor vinjorel }
$$

J.L. . W

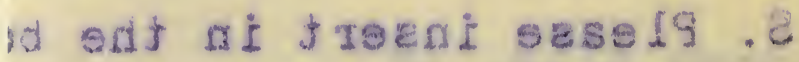

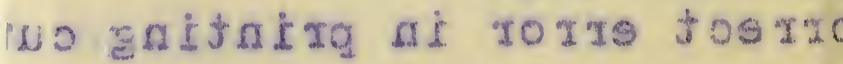




\title{
GHRISTIAN WIGOTAKLYY.
}

\section{WOONSPE I.}

\author{
WOONSPE WICOIE-CAJE.
}

Oekde. - Woyuha ota hee xni, tka tanyan cajeniyatapi kin he karniga wo."-Wicoie Wakan 22;1.

W. 'Toked eniciyapi he?

A. N. quisi M.

IV. Christian caje duha qa nakun akna-ci je wanj duha; akna-caje hin he totanhan duha he?

A. He ate caje qon ee.

W. Christian nicaje kin he tohand niçupi he?

A. Christian makaǵapi qon he ehayd.

IV. He tohaydtu he?

A. Miniamakaśtaypi qoul he ehald.

On-aspeyapi kin sani wicacaje waxto wan aknakapi, qa unma eciyatanhan ojuha wan mazaska ojuna akna yanka. Unma tukte i y otan tke he? Toka ca he iyotan terika he? Wakantàna he terida heon etanhań. $\mathrm{He}$ wowapi wan Wiconi Wowapi eciyapi yuha, unkan he wicacaje heca ojuna. Samuel ciqana, Joseph waxte, 'Timothy Koxkanaka, Wowapi Wakan ed on unvawapi kin, qa nakun ota wowapi kin he ed caje owicawapi.

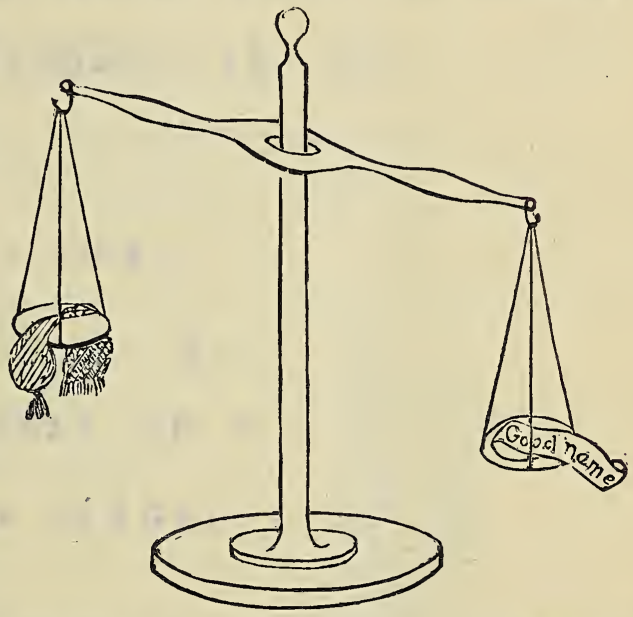

Woyuha ota yuhapi kta kuwapi kin eciyatahan on wicaxa ota tanyan cajewicayatapi kin toki erpekiyapı, exa tuktekted wicaxa nupin yuhapi heca wanwicunyakapi. Wakantanka lye cinca tona miniawicakaxtanpi kin owasin caje sdodwicaya qa wowapi tawa kin ed owicawa. Tona Iye anagoptanpi kta wicadapi xni kin henana caje wicapajujupi. He cinca wacinyepica' heca un wo, heced nicaje tawowapi kin ed ohinni un kta.

W ANYAKA PO.-Woiwange qa ayuptapi kin ihukuya iapi kagapi kin hena waonspekiya kin wakanheja kiciyawa, qaix ikceya okiyakin kta. 


\section{WOONSPE II.}

WOONSPE WICOIE-OKODAKICIYE WAKAN.

Oekcle._-“iakantanka tipi kage cin he niyepi."-i Kor. 3;9.

W. Wicacaje kil de tuwe nicu he?

A. Baptisma ed tona Waemiciciyapi hin; he ed Christ ematayhay, Wakaytanka cinca, qa mahpiya wokicolyze kin tawawayin kta. makağapi.

W. Waemiciciy api ehe cin he on toked yaka he?

A. Tona miye e Wakantanka takuku ecamon kta emici.iyapi kin. he?

W. Hena tohrind takuku ecanon kta keniciciyapi

A. Miniamakaśtanpi kin eciyatayhay on Wakintajka elta amahipi qon he ehand.

W. He ehrud Wakantanka taku ecanicon he? kağa.

A. Christ Okodakiciye Wakan tawa etanhay ma-

W. Nakun taku ecanicon he?

A. Ive r.inca teliidapi waun kta imacu.

W. Niçu kta keniciye ciq'n he taku he?

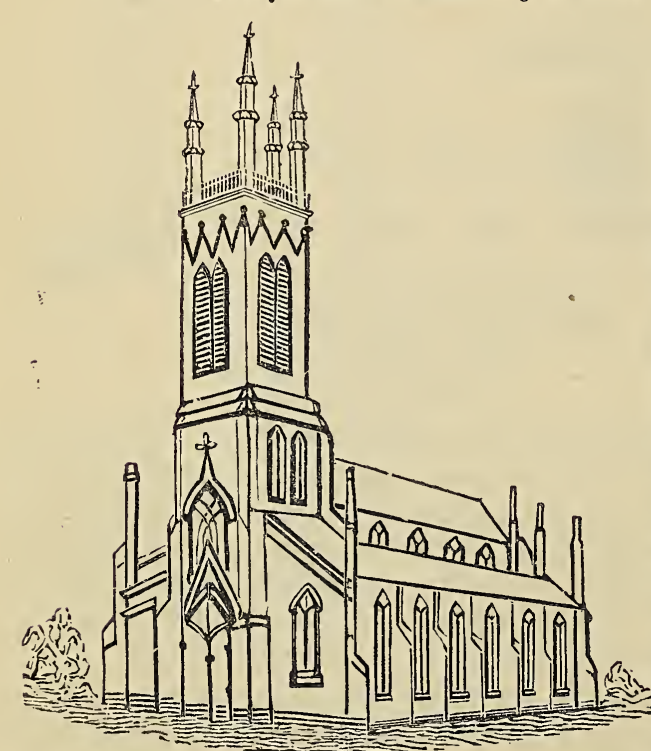

A. Malipiyata Ire ti kin ekta oyanke wayji.

Ded tipi wan owapi kin he taku tipi he? He taku on inagapi he? Exa Wakantanka tipi tokeca yuha qa he Iye Tipi-wakan Tawa eciya, unkan he inyan on kagapi kin h-na wicaxa qa winyan qa wikanheja koya heca. Tipi kin he ed inyan ciqana kin niye niwanji, qa nakun hokxiyopa otoiyohi miniakaxtanpi ecitayanhan on Wakantanka qupi kin hena koya hecapi, ecin minianicaxtanpi kin eciyatanhan on Christ Okodakiciye Wakan tawa kin ed opaniyanpi dakax 
heon hececa. Wukantanka tipi tawa kin ed inyan tankinkinyan qa ciqciqana ko yukan, nakun hunr owanyag waxtexte, qa ake hunr hena iyeced waxte xni; exa hena otoiyohi iye oyanke tawa rea ed yanka unkan hena tukte wanji kexa reyab icupi kinhan hetanhan on Okodakiciye Wakan kin iwaxakexni kta. Wakantanka cinca toked econpi kta iyececa kin eced econpi xni kinhan, inyan nasdeca qaix bduwahan aya iyececapi. Wakantanka Woniya waxte He inyan kin bduwahan qaix hinrpayapi kte xni okihiwicaya. Maka akan Wakantanka Okodakiciye Wakan tawa kin ed inyan xikxica of a yukan, exa Marpiyata Okodakiciye Wakan tawa kin he nina owanyag waxte qa suta qa owotanna heca kta. Maka akan Okodakiciye Wakan tawa kin ed Iye ikiyena yaun kta qa ded tanyan wowaxi ecayecon kta e oniciyin kta Christ icekiya wo, heced on Marpiyata Okodakicive Wakan owanyag waxte tawa kin ed oyanke oiyokipi wanji nitawa kta e heon etanhan.

\section{WOONSPE III.}

WOONSPE WICOIE-WOKICIZE.

Oekde.--"Wowacinye okicize waxte kin he ed econ wo."i Tim, 6;12.

W. He ehand Waeniciciyapi kin, niye on taku ecolppi he?

A. Micaje kin on taku yamni erapi qa hena ecerl ecamon kta keyapi. Tokaheya, wakayśica qa tohan kin owas 1), maka śice cin de ed taku woatakuniśni q: wowital kin, qa wicacelipi etanhal taku woalitani cal)tiheyapi kin owasin elipewayin kta.

W. Taku nicaje on ey api qa eced ecanon kta keyapi kin hena toyakeca he?

A. Yamni.

W. Taku eced ecanon kta keyapi tokaheya kin he taku onspeniciya he?

A. Takuku ehpewayin kta iyec ca yukay he e. e he?

W. Taku elipeyayin kta iyececa kin hena tukte

A. Wakayśica, maka kin qa wocantiheye xikxica mitawa kin.

W. Elipeyayin kta iyececa eyapi he on toked kapi he? 
A. Wakizin kta.

W. Minianicaśtanpi kin eciyatanhan on taku nicağapi he?

A. Christ taakicita.

Wiciteowapi wan ded kagapi kin he taku he? He taku wicoran yuha he? Hekta waniyetu ota qon he ehand akicita mazawakan takuna yuhapi xni tka ee wahukeza qa miwakan banska yuhapi, qa hayapi wan maza on kagapi heca kicunpi, heced tukted kicizapi ed opapi exa waxakayena ontonwicayapi kta okihi pica kte xni e heon etanhan. Maza wapaha helmet eciyapi heca on pa ataya akarpapi, qa sanpa anaicikxinpi kta on isto sani wahacanka ayapi. Tapete woakarpe wanica, ecin akicita wan kicizapi ayuxtan qa napa kinhan klepi exa heceı dakax. Wicaxayatapi kin ob iyaya, qa wowapetokeca wan on iye tawawicaya yutanin heca owasin wapetogwicatonpi. Christ taakicita henica. Minianicaxtanpi qon he ehand akicita obe tawa kin opaniya, unkan Iye tawaniya tanin kta on he ehand canicipawega itehu aoniwapi. Nakun on wakokipexti yaun kta e heon ozuye hayapi nicu.

Christ wicaxayatapi nitawa kin He wicaxa iyeced wokicize ecakicicon wo; wakanxica he kipajinyan, he taku xica econniciyin kta kuwa; maka kin de kipajinyan, to-

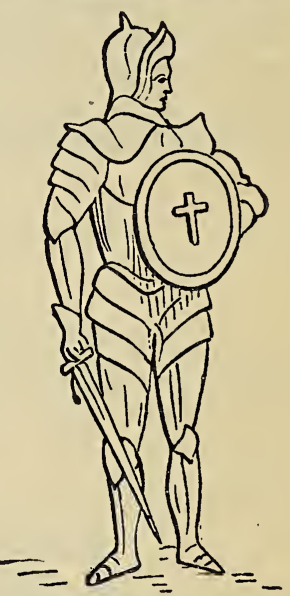
hand wakancekiya de kta qaix anpetu iyohi wowapi onsponiciciyin kta qaix wowaxi ecanon kta iyecece cin hena isanpa hayapi tekteca qaix wanapixkanye heca awacanni kta iyutanniyanpi kinhan; nix niye iyatayena nicipajinyan, tohand taku qevax etanhan nix onxpa iyotan tanka duha kta yacin, qaix tohand niyate qaix nihun toked econnixipi unkan hena anawicakigoptan xni niyecinka togye ecanon kta yacin kin hehandhand.

Akicita wacinyepica henica kta heced akicita oxpaye tawa kin tohinni erpeya nayajicin kte xni e oniciyin kta Christ icekiya wo.

\section{WOOONSPE IV.}

WOONSPE WICOIE-WICADA.

Oekde.- "Tuwe Wakantanka ed u kin he Wakantanka yukan wicada kta iyececa."-Heb., $11 ; 6$.

W. He ehand waeniciciyapi kin, niye on taku ecoupi he? 
A. Micaje kin on taku yamni eyapi qa hena ecer ecamon kta keyapi. Inoypa kin, Christ wacinranpi kin taku tonakiya Wicadaya Cajeyatapi kin hena swasin wicawada kta.

W. Waeniciciyapi kin ecanon kta keyapi inonpa kin he taku onsreniciva he?

A. Wicawada kta iyececa.

W. He taku e wicarada kta iyececa he?

A. Christ wacinyanpi kin, taku tonakiya Wicadaya Cajeyatapi kin hena owasin.

W. Christ wacinyanpi kin, taku Wicadaya Cajeyatapi kin hena tukted iyeuryanpi he?

A. Wowicada kin ed.

W. Wowicada kin ed taku tona Wicadaya Cajeyatapi kin hena tonakeca he?

A. Akenoipa.

W. Christ wacinvanpi kin, taku tona Wicadara Cajeyatapi kin hera toked wicayada kta iyececa he?

A. Micante kin ata! a on. hi he?

W. He wicayada kin toked duotanin kta oyaki-

A. Mii kin on epin kta owakihi.

W. Qa nakun tok d he?

A. Ni waun kin icunhan miolian kin eciyatayhay.

Wata-tanka akand iyadipi haronta on kagapi yuhapi kin, heca wan ded owapi. Iyadipi kin de aiydaipi tonakeca he? Han, akenonpa, qa Wowicada kin ed taku Wicadaya Cajeya tapi qaix Onxpa ix eya akenonpa yukan. Ho, heced iyadipi kin de Christ wacinyanpi kin, taku Wicadaya Cajeyatapi kin hena on takuku onspeunkiyapi kta hecinhan ūnkiyutapi kfa. Wata-tanka wan miniowanca ekta iyaye cinhan, anpetu owaxtecaka qa oxiceca koya iwiyeya un kta iyececa. He on taku toktokeca econpi kin, wata ihupa kin iyadipi haronta suta on kagapi heca ed iyakaxkr pi kta wanji ee, qa tuktekted mini nina rcin kaataja kin on tuwena wata owanka akand bosdad uajin okihi k ta iyecece xni, canna wata-tanka tahok xina wanji iyadipi kin ihupi inkpa ikiyena hehanyan adi yexipi, xina watopekiyapi kin hena toked kuwa kta qaix wata itokab toked wanke cin tanyan awicakicibdezin kta e he on etanhan.

Iyadipi kin nina cancan qa tate on kahunkunza exa sutaya 
oyuspa kinhan hinrpayin kte xni; tka ayuxtan kinhan minin hinrpayin kta.

Okodakiciye Wakan kin he wata-tanka wan iyececa, unkan he okna Wowicada suta wan tinsa unkoyuspapi kta e Wakantanka unqupi, wata-tanka wicaxa iyadipi tawa tinsa oyuspa kin he iyeced. Wowicada iya-

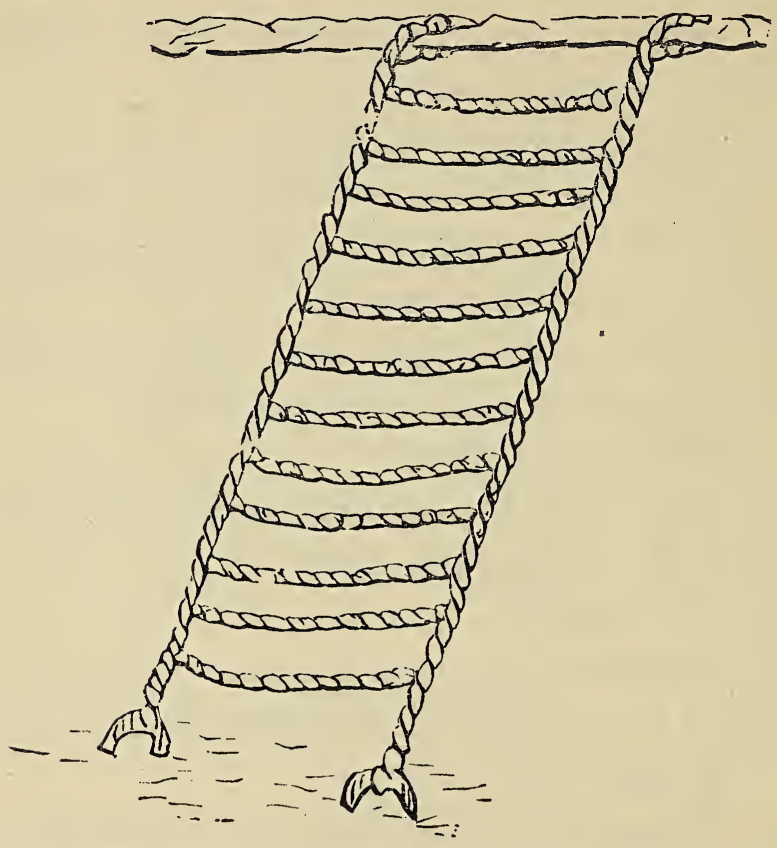

dipi kin de on Wakantanka ikiyena unhipi. Wakantanka Iye rea he unkicicagapi dakax heon yupsaka pica xni; tka Wowicada kin he unkayuxtanpi kinhan, "woiyokixica wiconi kin de ed miniknataja kin" hena ed minin untapi kta nriceca. Wakantanka cinca tawa kin wicada kta, qa wacantkiyin kta, qa Wowicada kin sutaya oyuspa un kta e okiyin kta.

Wana Wowicada kin ed iyadipi kin aiyadipi otoiyohi on taku Wicadaya Cajeyatapi kin hena unyawapi kta.

\section{WOONSPE V.}

WOONSPE WICJIE-ANAGOPTAN.

Oekde.- "Nitawoahope canku kin okna manimayan ye. Ps. 119;35.

W. He ehand waeniciciyapi kin, niye on taku ecoupi he?

A. Micaje kin oṇ taku yamni eyapi qa hena eced. 
ecamon kta keyapi. Iyanıi kin, Wakantayka tawacin wakall qa woahope tawa kin hena bduhe kta. qa tohalyaly wani kin hehajyan hena okna mawani kta keyapi.

W. Wakantayka tawacin wakan he on toked yaka he?

A. Taku toja ecamon kta cin kin hena owasin.

W. Woahope kin tonakeca he?

A. Wikcemna.

W. Wakantanka tawacin wakan qa woahope tawa kin hena tukteả iyeyaya he?

A. Wowapi Wakan kin ed.

W. Wakintanka tawacin qa woahope tawa kin duhe kta he on toked yaka he?

A. Wakayłanka toked econ maxi kin eced ecamol wakuwa kta.

W. Wakantalka tawacin qa woahope kin okna mayani kta he on toktd yaka he?

A. Wani kin hehayyan ajpetu iyohi Iye anawag่̀optan wakuwa kta.

Holixina wan te-
han omani yin kta ded owapi. Otonwe wan tehan, canku okna ye cin ihanke ekta wanu nyaka pi kin, he ekta ihuni kta cin. 'Tukted canku opa qon hed canicipawega wan pasdatapi kin he on canku okna yin kta iyececa kin hee e sdodya, canke ociqayena qa terika exa etan toka xni da. Wowapi ciqana wan yuha, qa toked ca ekde kta iyececa ed onspe-

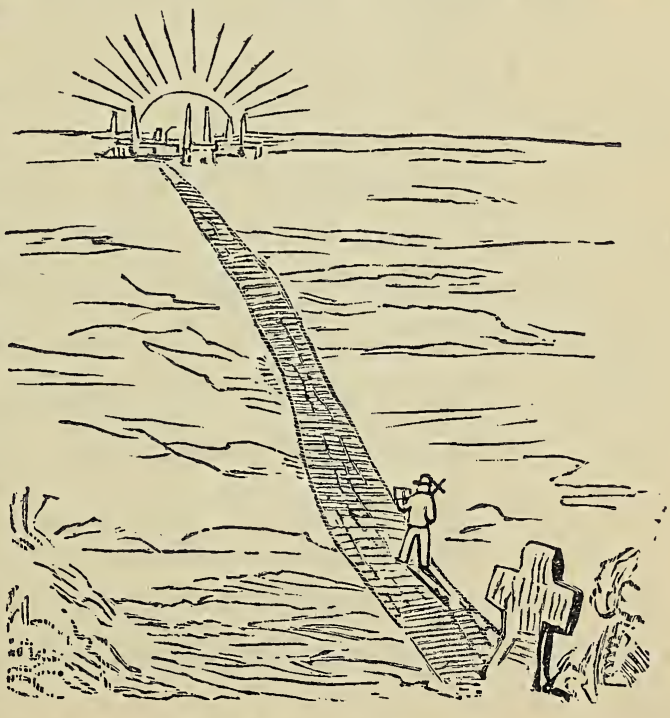
kiyapi kin he ijehan wanyaka. Hehan nakun canku kin 
etanhan icunom iyayin kte xni eawanikdaka, ecin anokatanhan wiwina, qa he ed iyayaye cinhan kdicu kta okihi kte xni kokipa. Otonwe kin ekta ihuni nin ecanni he? Tawowapi kin ed ijehan oton we kin he on yawa, qa ijehan etkiya etonwan, unkan ohanketa ekta ihuni kinhan toked nina rcin wiyuxkin kta he awacinyanked etkiya mani.

Nix eya tehan omani da. Wana idada. Otokahe tanhan idade ciqon canicipawega kin pasdatapi han, ecin minianicaxtanpi qon ehand canku marpiyata otonwe owanyag waxte tawa kin iyakdeye cin he okna Wakantanka siha enicikde. Canku kin owotanna qa ociqana; qeyax toked he okna mayani kta, qa toked icunon patanhan woartani maka rdirdina se wanke cin ed nihinrpeyin kte xni onspeniciyin kta e he on Wakantanka Wowapi Wakan nicu, ecin omayani kte cin de he "Tawoahope canku kin okna" ecanon kta heon etanhan.

Anpetu otoiyohi kin canku de okna mayani kta; unkan owihanke iyahuni kte cin on yani kin ataya ayakdusotin kta; exa hehand Wakantanka oton we wiyakpa kin ed inicu kta, qa bed nina cantewaxteya yaun kta qa otonwe canku oknakna hokxina qa wicincana nakun ota ob yaxkatin kta.

\section{WOONSPE VI.}

WOONSPE WICOIE-OKIYA.

Oekcle-"Omakiyapi kin he Itancan kin, marpiya maka ko kage cin he eciyatanhan ce." Ps. $121 ; 2$.

W. Waeniciciyapi kin is toked ecanon kta keyapi qon hena eced ecanon qa wicayada kta iyececa yadaka he?

A. Ho, awicakehay, qu Wakintanka omakire cin ol heced ecamon kta. Qia mahpiyata Ateunyanpi ki1, Jesus Christ Wanikiya unkitawapi eciyatayhay, wowanikiye de ed mico kin on awicakehal wopida ewakiya. Qa Wakantanka towaśte maqu kta icewakiya, heced tohanyan wani kin hehanyan he okıa waun kta.

W. Wakaytanka wokicize ecayecon kta, Iye wicayada qa anayaǵoptan kta, waeniciciyapi kin ecayon kta keyapi qon hena niyecinka eced ecanon kta oyakihi he?

A. Hiya, wamaxake śni. 
W. Heced taku ecanol kta keniciciyapi qon dena toked ecanon kta oyakihi kta he?

A. Wakantanka omakirin kta.

W. "Ho, awicakehar" eha, he on toked yaka he?

A. Ho, wicakese rcin.

W. "Wowanikiye" elie cin he on toked yaka he?

A. Okokipe wanica ouyyanpi.

W. He taku e eciyatalnal on wikope śni oulyanpi ed eniknakapi he?

A. Miniamakaśtanpi qon he e.

W. He toka e Wakantanka wikope śni ounyanpi kin de ed eniknaka he?

A. Jesus Itancan kin miye on tia he on etanhan.

W. Wakantanka towaśte kin he on toked kapi he?

A. Wakantanka tookiye.

Woonspe de itokab ehake kin ed tehan omayani kta wan nitokab wanka ociciyaka, he yani kin ihuniyan kipi kta,unkan canku

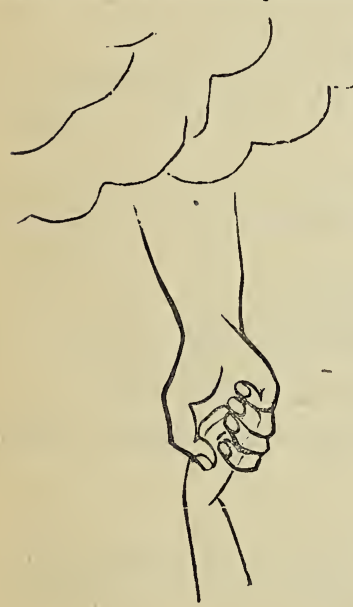
okokipe wanin ed eniknakapi kin he anokatanhan wiwina maka rdirdina yukan heced epa. Qa tuwena oniciye xni, nixnana canku kin de okna idade cinhan nina terika iyeyayin kta; tra Wakantanka, ohini awanniyaka qa ihanikte cin, He de sdodniciciya unkan nihinrpayin kte xni qaix canku icunom wokokipe ekna idanin kte xni, tha ee canku kin okna wikopexniyan yus aniyin kta e on isto waxaka tawa kin marpiyatanhan kudkiya niciyugata.qa nape waxake xni nitawa Iye nape kin on oniyuspa. Heced oniciyapi kin de he Wakantanka towaxte nicu kin hee. He niye kici wicotakuye tawa kin hee. Wakantanka ohini nape niciyugad un, oniciyin kta e heon etanhan. Nape nitawa Iye nape kin etanhan iyekcu kinhan, okini canku yaknuni qa tohini marpiyata iyahunni kte xni naceca; exa hecanon kin on icantenixica, unkan ake nape yecidugate cinhan, He ake yus aniyin kta e ohini wiyeya un heced iyeyayin kta.

Wokicize tanyan ecanon kta e oniciyin kta. Wowicada iyadipi kin sutaya oduspin kta e oniciyin kta, Iye anayagoptan 
kta e oniciyin kta. Iye nape kin ixta on wandakin kta oyakihi xni, exa Iye ceyakiyin kta oyakihi, unkan oniciyin kta e on Iye nikiyena un kta.

Iye heced tenirida kin on wopida eyakiyir kte xni he?

\section{WOONSPE VII.}

AKTA YAWAPI-CHRISTIAN WICOTAKUYE KIN.

W. Okodakiciye Wakan Wiwicawangapi kin ed onxpa tonakeca he?

A. Zaptan.

W. Onxpa tokaheya kin taku eciyapi he?

A. Christian Wicotakuye kin.

W. He on toked kapi he?

A. Miniunkakaxtanpi kin eciyatanhan on Wakantanka wowaxte ecaunkiconpi kta keunkeyapi, qa unkiyepi kin Iye anaungoptanpi kta unkekiyapi.

W. Wicotakuye kin de iyowajapi kin hena tuwepi he?

A. Tona miniawicakaxtanpi kin hena owasin.

W. Christian nicaje kin okdaka wo.

A. -

W. Woyuha ota isanpa teridapi kta iyececa kin he taku he?

A. Tanyan cajewicayatapi kta.

W. Wakantanka cinca tawa cajepi kin tukted owa knaka he?

A. Wiconi Wowapi kin ed.

W. Minianicaxtanpi kin eciyatanhan on he taku e ed opaniyanpi he?

A. Christ Okodakiciye Wakan tawa kin.

W. Wakantanka cinca kin hena taku iyececapi he?

A. Wakantanka tipi tawa kin ed inyan kin hena.

W. Hed oyanke nitawa kin ed, waxte qa wacinyepica nicagin kte cin He tuwe he?

A. Woniya Wakan kin.

W. Minianicaxtanpi qon ehand taku eced ecanon kta keniciciyapi qon tokaheya kin he taku he?

A. Christ taakicita waxte wan iyeced, woartani wakizin kta.

W. Eced ecanon kta keniciciyapi inonpa kin he taku he?

A. Wowicada kin he sutaya yus waun kta.

W. Wowicada kin he taku iyececa unkeyapi he?

A. Iyadipi wan. 
W. Eced ecanon kta keniciciyapi iyamni kin he taku he?

A. Wakantanka tawoahope canku kin he okna maxani kta.

W. Taku kin dena ecanon kta iyececa yadaka he?

A. "Ho, awicakehan; qa Wakantanka omakiye cin on eced ecamon kta."

W. Wakantanka tookiye kin he taku iyececa he?

A. Wicanape waxaka wan, canku marpiyata iyakdeye cin okna yus amayan.

WanAKya Po.-Akta Yawapi kin de hanska sececa kinhan, nonpa kiyuxpeya onspewicakiyapi waxte.

\section{ONXPA II. GHRISTIAN WOWIGADA.}

\section{WOONSPE VIII.}

WOONSPE WICOIE-WOWICADA. Jude, 3.

Oekde.-"Wowicada kin he kitanye rcin yakitanpi kta."

W. Wowicada nitawa Woeye kin eya wo.

A. Wakantanka, Ateyapi Iyotanwaśake cin malipiya maka iyakna Kağe cin, he wicawada:

Q. Jesus Christ, Cinhintku hecena, Itancan unyanpi kin; He Woniya Wakan eciyatanhan icaǵa, Witayśna un Mary etanhan torpi; Pontius Pilate kakiśya, Canicipaweğa ed okatanpi țe ça hapi; Kuya hades ekta i, lyamnical wicațe cin etayhay ake kini; Wankıり mahipa kin ekta iyaye, ça Wakantanka, Ateyapi Iyotal-waśaka etapa kin eciyatinhan iyotanka; Heciyatanhal wiciśa nipi na țapi kin wicayaco u kta.

Woniya Wakal kin he wicawada; Okodakiciye Wakan Owancaya kin; Wakanpi Odakodkicịapi kin; Woartani kajujupi kin; Wicataycan kini kte cin; Qa Wiconi owihanke wanice cin. Amen.

W. Creed eyapi kin ol toked kapi he?

A. Wowicada kin.

W. He ed taku oyakapi he?

A. Taku wowicake wadakin kta iyececa kin hena.

W. Wowicada kin he tukte tajhay uyy uhapi he? 
A. Okodakiciye Wakan kin he he unqupi.

W. Woonspe tawa kin hena owasin tukted iyeunyanpi kta unkokihipi he?

A. Wowapi Wakan kin ed.

W. Toked num kta iyececa he?

A. Mii kin on epin kta iyececa.

W. Nakur toked nur kta iyececa he?

A. He wowicake wadaka se iyeced mikduha kła iyececa.

W. Heced ikduhapi qa ipi kin on taninyan eyapi kin he taku eciyapi he?

A. Wowicada kin okdakapi.

W. Christian tokeca heconpi ece tka qon he?

A. Ho, nakuij wanjikji henฤ țerwicayapi eśa iıihanśui econpj.

W. Tona heconpi qon taku ewicakiyapi he?

A. Martyrs.

Wayawawicakiyapi ekta wakanheja taku wanji ohiyapi kta kuwapi canna he yuhapi kta kitanpi unkeyapi tuktekted. Woeye kin he oekde nitawa kin ed un he? Taku wanji icupi kta e on nina xkinciyapi he heced kapi; qa taku wanji teridapi unkan he yuxicapi xniyan kduhapi kta e on wokicize econpi heced tuktekted nakun kapi. Akicita wokicize ed yapi canna, wanji wowapi wiyoki. hena, qaix wowapi owanyag waxte, akan makoce tawapi wiyacinpi

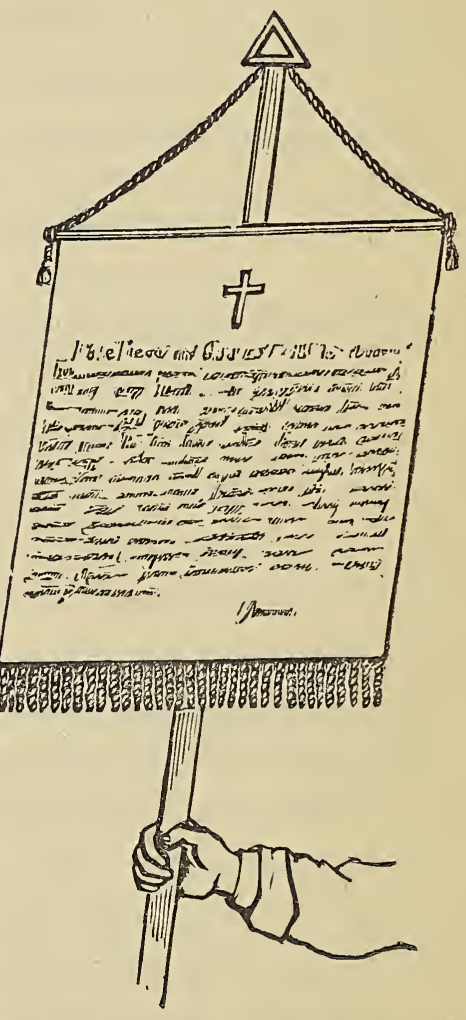
tawa qa iapi karnigapi tawa oowa wiyakpakpa on owapi heca wan yuha mani, heced akicita kin ijehan he ekta etonwanpi kin on makoce teridapi kin he kiksuya hinkdapi qa nakicijinpi kta on sanpa cante tinsyaked kicizapi ed xkanpi kta e he on etanhan. Wowapi kin he erpeya napapi qa tokawica- 
yapi kin he wicakipi kta ecaca tawatedyapi kte xni, qa akicita wan he aye cin on ta exa cona kikda kte xni. Akicita hececa kin wacinyepicapi, ci?

Christ taakicita henica, unkan wowapi ekdepi tawa kin ihukuya wokicize waditagya ecanon kta iyececa. He akand canjcipawega kin wiyakpa tanin, qa Wowicada kin hed owapi. Tuwe iyotan kici tokayeciye cin, wakanxica kin hee, iye taku teyarida kin de nici kta cin, qeyax on nita exa he expeyayin kta iyececa xni. Christ taakicita Wowicada kin de nakicijinpi kin on wokicize econpi tka qon, qa wanjikji heconpi kin icunhan iyuxkinyan tapi.

He tanyan onspeniciciya qa on toked kapi kin tanyan sdodyayin kta iyececa, qa nicante on wowicake yadakin kta, kinhan Wowicada wan Wakantanka unqupi qon he, Iye oniciya kin on, "kitanye rcin yakitan kta" e wiyeya yaun kta.

\section{WOONSPE IX.}

WOONSPE WICOIE-TRINITY.

Oekde.-."Ateyepi kin, qa Cinhintku kin, qa Woniya Wakan kin caje on." Matt. 28;19.

W. Wowicada Woeye nitawa kin dena, etanhay taku tonakiya iyotan unnispe he?

A. Tokaheya, Wakantanka Ateyapi kin He wicawada kte cin, he onmaspe, He miye qa maka kin owasin kağe cin Hee. Inoypa kin, Wakantayka Cinhinłku kin, He miye qa wicaśa owasin opeunkitolpi kin Hee. Iyamni kin, Wakaytanka Woniya Wakay kin, He miye qa Wakantanka taovate kin owasin unyurakaypi kin Hee.

W. Trinity woeye kin he on toked kapi he?

A. Yampi ed wayjipina.

W. He taku onspeunkiyapi he?

A. Wakantanka wanjina, exa Iye ed Tuwe kin yamnipi.

W. Trinity kin ed Tuwe kin Tokaheya kin $\mathrm{He}$ toked eciyapi he?

A. Wakantanka Ateyapi ki).

W: Tuwe kin Inolppa kin He toked eciyapi he?

A. Wakantanka Cinhintku kin. 
W. "He opeunkitonpi" kehe cin, he on toked yaka he?

A. Unnipi kta e heon Iye we tawa kpapson.

W. Tuwe Iyamni kin He toked eciyapi he?

A. Wakantanka Woniya Wakan kin. yaka he?

W. "He unyuwakanpi" kehe cin, he on toked

A. He waśte unkagiapi.

W. Dena Wakantanka yamnipi he?

A. Hiya, Tuwe kin, yamuipi eśa Hena, Wakaytanka wanjipina.

W. He okiyakahiniga ovakihi he?

A. Hiya, eśa wicawadi kta owakihi, Wakantanka Jye heya heol etanhay.

W. Hetoka e Trinity kin wicada onnispe kta iyececa he?

A. Caje Wakan kin $\mathrm{He}$ ol miniamakaśtanpi kin heorl.

Tohinni maxtin-tawote ape wanji duxpi he? Hehan dujuju qa onxpa yamni kin hena toked ape yamni iyececa sececa

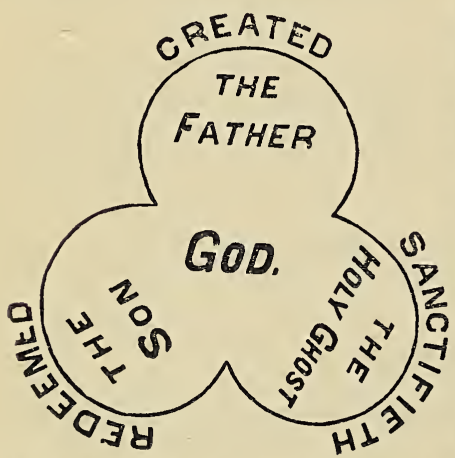
kecanni he? Ded taku owapi kin he maxtin-tawote ape iyecera he? Ho, heca taxpu yuksapi iyececa. Tipi-wakan ed heca ota tuktekted wandakapi naceca. He toka e Tipi-wakan itimahed heca ekdepi huwo. ecanni he? Ecin, ape kin hena 'Trinity kin on woonspe waxte qa wakan onspeunkiyapi kta iyokihi kin heon etanhan. Ape ciqciqana yamni hena ape wanjina kin he iyeced Ateyapi kin, qa Cinhintku kin, qa Woniya Wakan kin hena Tuwe Yamnipi exa

Wakantanka wanjipina kin he onspeunkiyapi. Tuwe Yamnipi kin dena otoiyohi niye etkiya nina waonxida qa waxte. Wakantanka Ateyapi kin Iye icarniyan, qa ohinnı awanniyaka. Wakantanka Cinhintku kin Tye we tawa kin on, wakanxica towaxake kin etanhan, openiton; niye qa wicaxa owasin on canicipawega akand kakija qa ta qonband we kpapson qon he ehand. Wakantanka Woniya Wakan kin Iye nicante kin 
ed woawacin waxtexte nicu, qa niwaxte kta e heon anpetu yohi oniciya. Nicante ataya on de wicayada, qa Wakantanka wakanheja waxake xni wan heced taku ota ecakicon kin $\mathrm{He}$ ijehan wopida yaqu kta iyececa.

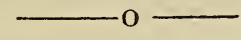

\section{WOONSPE $\mathrm{X}$.}

\section{AKTA YAWAPI.}

W. Okodakiciye Wakan Wiwicawangapi kin onxpa inonpa kin ed taku cajeyatapi he?

A. Christian Wowicada kin.

W. Wowicada eyapi kin, he on toked kapi he?

A. Taku wowicake wadakin kta iyececa kin he.

W. Wowicada kin he taku iyacinpi he?

A. Wiyokihena wan.

W. Christ taakicita kin he on toked econpi kta iyececa he?

A. 'Tuwepike ceyax owasin he wanyaka okihipi kta e heced ayapi kta.

W. Nakun taku he?

A. Sutaya oyuspapi kta iyececa.

W. Qa nakun taku he?

A. He wokicize ed nakicijinpi kta e wiyeya unpi kta iyececa.

W. Nix niye kin heced ecanon kta iyececa he?

A. He, ecin Christ taakicita hemaca dakax.

W. Wowicada kin etauhan taku iyotan onnispe kin he taku he?

A. Trinity Wakan kin wicawada kte cin hee.

W. Trinity kin He taku iyacinpı he?

A. Maxtin-tawote ape wanji.

W. Maxtin-tawote ape kin onxpa tonakeca he?

A. Yamni.

W. Trinity Wakan kin ed Tuwe kin tonakecapi he?

A Yamnipi.

W. Wowicada kin ed nakun taku onspeniciyapi he?

A. Okodakiciye Wakan kin wowicake okna wicawada kta.

W. Wowicada kin etanhan nakun taku onnispe he?

A. Wakantanka woartani kicicajuju kte cin he. 
W. Qa nakun taku he?

A. Tancan unkitawapi kin wicarapi kin etanhan piya kinipi kta.

W. Tancan unkitawapi kin piya ekicetuyapi kta he to. ked tanyan sdodunyanpi he?

A. Christ kini kin he eciyatanhan on. he?

W. Nakun taku wowicake yadakin kta hetanhan onnispe he?

A. Marpiya ekta wiconi wan owihanke wanicin kte cin

W. Wiconi wowiyuxkin ojuna kin he ohiyeunkiciciye ciqon He tuwe he?

A. Jesus Christ Itancan unkitawapi kin.

\section{ONXPA III. GHRISTIAN ORANPI.}

WOONSPE XI.

WOONSPE WICOIE-ORAN.

Oekde.-"Wakantanka kokipa wo, qa Iye tawoahope kin ahopa wo." Eyanpaba 12;13.

W. Waeniciciyapi kin, Wakantanka Woahope tawa kin hena ahoyapin kta, woiçiconze enicicisapi keha. Hena ton:akeca omakiyaka wo.

A. Wikcemna.

W. Tona ee he?

A. Kdinapapi Wicowoyake iwikcemna-noypa kin ed, hena Wakaytalka eya qa heya, Iiancan Wakantulka nitawa, Eg!pt makoce etayhay, wayaka tipi etayhay, inapeniye cin He miye.

W. Woahope kın dena tokaheya owapi qonhand he taku e akayd owapi he?

A. Ijsal bdaska noipa.

IV. Hena toked owapr he?

A. Wakintanka napsukaza tawa kin he ol. 
W. He tuktedtu e Wakantanka Moses hena qu he?

A. Re wanji Sinai eciyapi kin hedtr.

W. Hena tuwepi e Wakantanka Moses onspewicakiya ṡi he?

A. Israel cincapi kin.

W. Tuwe tokeca nakun iyowaja he?

A. Ho, tuweke çeyaś owasin iyowajapi.

W. Niye hena ahoyapin kta iyec ca he?

A. Ho, owalhall kta ivececa kin hee?

W. Woahope invan bdaska tokaheya kin akand owapi kin h 'na tona ee he?

A. Topa tokaheya oyakapi kin hena ee.

W. In yal inon pa kin akand owapi kin hena tona ee he?

A. Sं ıkpe ehake oyakapi kin heńa ee.

Ite owapi wan ded kagapi kin ihukuya caje owapi kin he taku caje be?

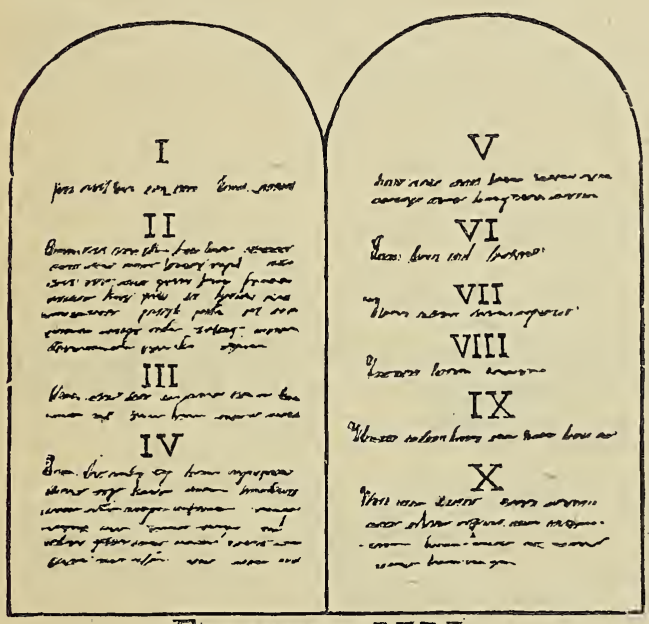

EXODUS XX.

Wowapi Wakan ed wowapi inonpa kin he caje kin ee, unkan he ed wicowoyake iwikcemna nonpa kin ed unkodepi kinhan Woahope Wikcemna kin hed iyeunyanpi kta. Exodus eyapi kin he "kdinapapi" heced kapi, qa oyate wan Egypt makoce ed te. han wayaka unpi qa nina kakijapi, tka hetanhan kdinapapi qon hena woyakapi tawapi wowapi kin he ed unkokiyakapi. Wakantanka tokedked yusawicaye cin hecekced okna ikdaka omanipi, makoce owanyag waxte wan, Canaan eciyapi, ekta ihunnipi hehanyan. Wicitokab miniowanca wanka; exa Wokantanka he kiyuxpa, qa 
mini kin conkaxke iyeced anog bosdad inajinkiya, heced maka puza akand mani koakatanhan yapi kta okihipi. Hetanhan canku okna yapi kin makoskantu wan tanka opta rp:ya; exa ake Wakantanka, anpetu canna marpiya bosdad han heca wan okna, qa hanhepi canna peta bosdad han okna, wicitokab iyaya, heced canku nunipi kte xni e heon elanhan. Marpiya kin he, qaix peta kin e exa owanji najin kinhan, wakeya r.kepi qa okna etipi. Taku yutapi kła wanica secec qonhanhand, Wakantanka woyute marpiya etanhan on wowicaqu, qa yatkanpi kta e on inyan suksuta etanhan mini hiyawicakiciya. Heced omanipi qon icunhan anpetu wanji re wan tanka, Sinai eciyapi, ekta hipi unkan hed tehan owanji yankapi. Wakantanka Mnses re inkpa ekta kico, unkan inyan nonpa Woahope akand owapi kin hena qu, qa Israel cincapi kin hena onspewicakiya xi, Iye toked tawacin sdodyapi qa eced econpi kta e heon etanhan.

'Tehan ikdaka omanipi qon he ohanketa ihunnikiyapi: ecin Wakantanka Canaan ekta tanyan awicahi, qa hed maga ojupi qa tipi icicagapi, dakax makoce wan Wakantanka wieaqu kta kewicakiye ciqon he wana ed ahiyotankapi kin heon etanhan. He tokae Wakantanka hena tewicarida qa awanwicayaka he? Hena Iye Okodakiciye Wakan tawa qa Wicotalsuye tawa kin he epi dakax.

Nix eva Wakartanka Okodakiciye Wakan tawa kin oyapa, qa nix eya Wakantanka Woahope tawa kin ahoyapin kta iyececa, unkan Iye tenirida kta. qa cinca wan teridapi kin iyeced, awanniyakin kta, qa maka kin de opta ikdaka se omayani kin icunhan, qa makoce wan nicu kta keniciye ciqon, arpiya makoce kin hee, e ekta ihunniyan tanyan yusaniyin kta e he tar yan sdodya yaun kta lyececa.

\section{WOONSPE XII.}

\section{WOONSPE WICOIE-WAKANTANKA。}

Oekde.- "Itancan kin Wakantanka tanka kin heon, ho, taku wakan owasin iwankah Wicaxayatapi tanka un." Ps. 95;2.

W. Woahope tokaheya kin eya wo?

A. Mitokan taku wakay tokeca duhe kte śni.

W. Wicoie way on Wakaytanka toked waśake cin onspeunkiyapi kin he tukte e he?

A. Iyotay-waśaka.

W. Wakanłanka towaśake kin toked ikdutanin he? 
A. Miye makağa, qa maka owasin kağe cin he on. ka he?

W. Wakantanka niye qa maka owasin away:a-

A. Ho, hecol.

W. Woahope tawa kin hena toked ahoyapin kta ojakihi he?

A. Wakantanka wicawada kin he eciyatayhay on.

W. Wakantanka heced tanka qa waśte dakas̉ heon etanhan taku itonyapin kta iyececa kin he tukte e he?

A. Iye iyokipiwayin kte śni kin he itonwapin kta iyececa kin ee.

W. Iye waśte dakaś heon etayhay niś toked oyahil kta irececa he?

A. Micante kin ataya on cantewakiyin kta.

W. Iye canteyakiye cinhan toked he yakdutanin kta oyakihi he?

A. Woahope tawa eced ecamon kta e aimiçiciye cij ( 1 .

Watexdake kicunpi ece kin, hena taku wicaxapi he? Hena wicaxayatapi-hecapi, ci? Qa wicaxayatapi wanji watexdake kicunkiyapi eca, tanyate kin iyuxkinpi qa wowiyuxkin tawapi otakiya kdutaninpi ece. Wacipi,qa dowanpi, qa wotapi, qa wicaxayatapi kin iye wicaxayatapi-oiyot a $\mathrm{ke}$, oiyotanke iyotan wankantu ya qa waxte he heced

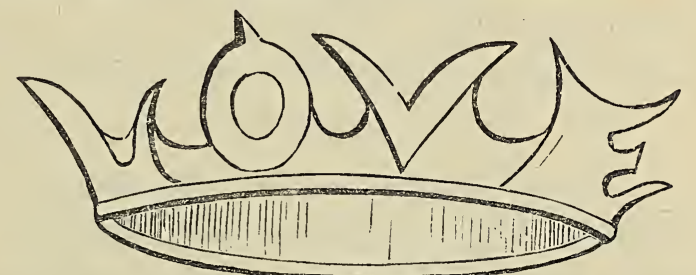

kapi, heca wan akand iyotankehan yanka. Eya, heced wicaxayatapi oiyotanke wanji akand iyotankeunkiyapi kta e, qa watexdake kicununkiyapi kta wicaxayatapi makoce kin de ed takuna unyuhapi xni ce ehapi kta naceca. Exa Wicaxayatapi wanji tanka qa tawaunyanpi yukan, qa otokaheyatanhan wicaxayatapi unpi tka qon hena owasin etanhan tukte wanji ke ceyax he isanpa De nina tanka, qa waonxida, qa waxte. He unkiye nina rcin teunridapi. He wandakin kta yacin he? 
Eya, dehand niixta kin wowitan tawa ayutin kta ecaca iyokihi xni, exa takomni anpa wan hi kta ed awicakehan Wicaxayatapi kin toowanyag waxte rea kduha un ixta on wandakin kta. Unkan dehantu kex watexdake yaqu kta oyakihi wánji yuke do. He taku he, eha he? Ecin, tokaheya loyal. wacinyepica he kapi, heced yaun kta. Loyal eyapi kin e'd oowa tokaheya kin he taku eciyapi he? L. Ho, watexdake kin ed

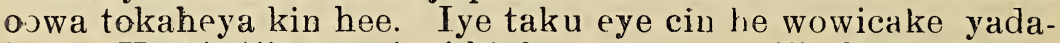
ka, qa He kipajinyan aiapi kinhan niye nayecijin kta he hered kapi. Watexdake kin ed oowa iyokihe kin he $O$ ee. He obedient, waanagoptan he kapi, qa he wicoio kin he eokiya eknakapi. Iye toked econ nixi kinhan iduxkin qa ecercin ecanon ece, he heced kapi. Oowa iyamni kin he $\mathrm{V}$ ee, qa venerate, yuonihan he kapi, qa he wicoie kin he eekiya eknakapi. Iye itokab canpexka makakde erpeniciya, qa Iye cajeyatapi kinhan aiyukcanyan inina yaun ece he heced kapi. Oowa thake kin he $\mathrm{E}$ ee, he esteem, iyotan-dakapi he kapi qa he wicoie kin he eekiya eknakapi. De taku kin niyukan kinhan, taku owasin isanpa Iye qa Iye toked iyukcan kin hena nina awacani, qa wicaxa tokeca Iye waxtedakapi kta yakuwa ece. Unkan oowa kin dena owasin witaya eunkdepi kinhan watexdake wan Wakantanka, Wicaxayatapi Tanka ree cin Hee, unkiye otoiyohi Iye unqupi kta cin kin he kagin kta. Love (wocantekiye) he watexdake kin ee, he uncantepi kin hed ecena etanhan hiyu okihi kin, heced on Iye hed oiyotanke iyotan wakantuya qa waxte kin he tawa kta.

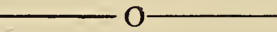

\section{WOONSPE XIII.}

\section{WOONSPE WICOIE-WAKAGAPI.}

Oekde._"Hokxiyopa cikciscinpina, taku wakagapi kin itehan ikduha po." 1 St. John 5; 21.

W. Woahope inonpa onśpa tokaheya kin eya wo.

A. Wakağapi wanjina niçicağin kte śni, nakun wajkald mahpiya ekta, qa ihukuya maka kin ed, qa maka kin ordateya mini kin mahed, taku hiyeye cin, wanjina oyakağin kte śni. Wicitokab wicayakipatuje, ça cewicayakiyin kte śni.

W. "Taku wakağapi" eyapi kin he on toked kapi he?

A. Cay, qaiś inyał, qaiś taku tokeca es̉a etanhan takuke çeyaś ouncag̀e tawa kin akiyececa wayji kağapi. 
W. "Taku hiyeye cil, wayjina oyakağin kte šni" eyapi kin he on toked kapi he?

A. Takuku iteowapi unkağapi kte s̉ni.

W. Hoks̉in-kag̉api yuha s̉katapi kta e, qais̉ iteowapi ikce wayyakapi kta e on kaǵapi kinhay hena wicolian sica he?

A. Hiya.

W. Wicohan sice cin he tukte e he?

A. Hoks̉in-kağapi qais̉ iteowapi es̉a itokab kipatujapi kta e, qais ohodapi kta e on kaýapi.

.W Taku okağapi ohodapi kta e on kağapi kin hena toked eciyapi he?

A. Wakağapi.

.W Wankand malipiya ekta hiyeye cìn, qa hena okağapi qais̉ iteowapi kag̉api kta okihi pica kỉ hena tona ee he?

A, Oknikde-wakanpi kin, qa aypetu wi, qa hayhepi wi, qa wicanlipi kin.

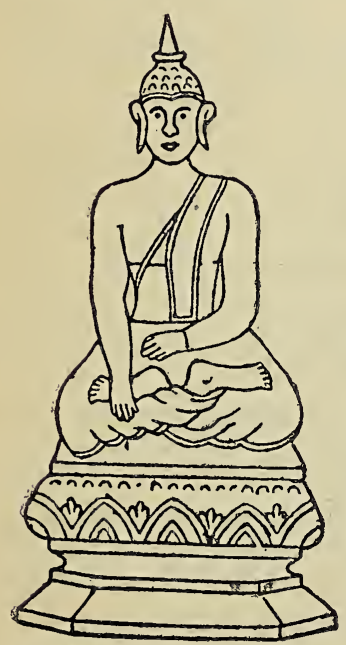

.W Ihukuya maka kin ed tona ee he?

A. Wicaśa qa wamakaškay.

W. Maka kin olidateya, mini kin mahed tona eehe?

A. Hoğan.

Iteowapi wan ded kagapi kin he wakagapi iteowapi heca. Ehanna oyate wan tanka tia onxikapi, qa Tuwe Ixnana Wakantanka wicakapi kin He on taku na sdodyapi xni, unkan hena wakagapi kin de itokab canpexka makakde inajinpi qa ohodapi tka qon. Oyate wakagapi ohodapisa kin, hunr kaked okna wakagapi icicagapi ece: tukted canxoka kin can wekna iyayapi qa can wanji tanka kawankapi, qa hetanhan adetka kin hunr on cetipi, kadiciyapi kta $\mathrm{e}$ he on. Hehan ake hetanhan adetka kaksapi qa on aguyapi qaix tado xpanyanpi. Qa can wan heced 
wowidngyapi exa hee rea etanhan ake onxpa icupi qa on wakagapi owanyag xica wan icicagapi qa he itokab makata erpeiciyapi, qa awanwicayin kta e icekiyapi, qa taku woyuha iyot m teridapi kin etanhanhan qupi. Eya, Wowapi Wakan kin ed he on sanpa niciyawapi kta naceea. He woyakapi kin Isaiah 44; 13-20, hed owapi.

Nix niye kin wakagapi wanji tukted yaknaka he? Icin, Oekde kin, qa Woahope kin hena nupin ed heca takuna duha kte xni eniciyapi e he sdodyaye do. Heca takuna duhe xni tanyan sdodyakiya kecanni, exa okinni ciqana wanji tukted niyukan naceca Ito owade kta e iyowinmalkiya ye; okinni iyewayin kta. Eya, sicanopiye nitawa kin ed owade exa etanhan toka kte xni, qeyax opiye tokeca wanji niyukan qa hed janka naceca. Wakantanka he ed taku hiyeye cin owasin rein tanye rcin abdeza. Nicante kin he e he? Ho Okini wakagapi wanji waxteicidapi eciyapi kin, he hed un naceca; he olinni taku qeyax etanhan wanji iyotan tanka qa waxte kin he iye yuha kta cin ece. Nakun wakagapi wanji witantanpi eciyapi kin iyeunyanpi kta seca, hayapi waxtexte qaix ite owanyag waxte tawa kin iwinkta ece kin hee. Qa okinni nakun wakagapi wan tanka, iyokipiiciyapi, hed un naceca; Wakantanka qaix niyate qa nihun teniridapi kin hena iyokipipi xni exa takomni niye toked iyonicipi kin eced ecanon kla yacin rce cin he bee. Wakagapi unkiiarvapi kin hena takuke ceyax unkicantepi kin etanhar reyab erpewicuny+upi kta e on Wakantanki ounkiyapi kta e iceunkiyapi kta iyececa, icin tohanyan wakagapi kin hed onnyanpi kimhan Wakantanka Iye unkicipi un kta okibi xni kin heon etanhan.

Taku on hececa kin, he tokata Anpetu-wakan ehand ociciyakapi kta.

\section{$\mathrm{O}-\cdots$ \\ WOONSPE XIV. \\ WOONSPE WICOIE-WOOHODA.}

Oekde.-- Itancan Wakantanka nitawa kin he ohoyada kta, qa ixnana wowidagniyin kta." Matt. 4;10.

W. Woahope inolpa olśpa inompa kin eya wo.

A. Wakantalka nitawa, Italcal kil he Miye, Wakaytanka winawizi kiı hemaca, qa tona śicemadapi kin atewicayapi wahtanipi kin, iye cincapi kil ekta awicawai ece, wicoicağe yamni qa topa hehayyay; qa tona waśtemadakapi qa mitawoahope yuhapi kin, hena kektopawing่̀e ota ed wowaonsida bdutaıin. 
W. Wakantanka winawizi eyapi kin he on toked kapi he?

A. Iye kin ośtan taku tokeca waśteundakapi qa ohoundapi kinhạ išihda.

W. Wakantanka heced winawizi kta he hecetu he?

A. Ho, icin Iye ußkag̉api, qa taku qeyaś Iye hena owasin ecaunkicicoljpi.

W. Wakantanka tona woahope tawa kicaksapi kin, hena toked ecawicakicon kta he?

A. Kakiświcayin kta.

W. He on nakun toktokeca icakišyapi kta he?

A. Ho, iye cincapi qa takojakpakupi kiø.

W. Wowaonsida eyapi kin he on toked kapi he?

A. Wacaytkiyapi nais̉ waonśidapi.

W. Wakantanka wowaoıśida wicakiyutanin kte cil hena tuwepi he?

A. Tona Iye išnana waśtedakapi, qa wawokiya ṡkanpi kiı.

Ninihruha kinyankiyapi nina waxte wanji nicicaga, unkan hokxina tanka wan he kohan nici qa iye tawa se yuha xkata ehantanhanx, Lina icantenixicin kta, ci? Wicincana kin dena nanji wowapi-iwapetokeca icicaga hantanx, unkan wicincana tokeca he iye yuha kta icu kinhan, he hecetu kta he? Niyate iye oti kta e tipi waxte icicaga, unkan wicaxa tokeca he woyuha tawa kduha ikdaka ed ahi qa oxtan ed ounyan kinhan, niyate iyokipi kta he? Hiya, ecaca iyokipi kte xni. Tipi wan niyate icicage cin he iye tawa, qa miniruha kinyankiyapi qaix wowapi-iwapetokeca qon hena hokxina qa wacincana hena kagapi qon iye tawapi.

Ho, unkan ito, nix niye kinhan, tancan wowinihan kagapi nitawa kin koya, tuwe nicaga he? Ninape on taku oduspa qa anin kta oyakihi kin, nisiha dus inyankin kta okihi kin hena, niixta wiyakpakpa qa ninoge wanaron kta oranko kin, qa hena owasin isanpa cuwi mahed nicante wacantkiya qa waihaktesa kin, nitawacin bdiheca qa taku ota onspe kta okihi kin, qa ninagi Wakantanka ohoda kta okihi, qa nitancan kin a aya He wawokiya xkankiyin kta nalkun okihi kin, hena tuwe kaga he? Wakantanka Iye hena owasin kaga, icin Wakantanka nicaga dakax, qa lye tawaniyan. 
Wowaxtedake nitawa qa wawokiye yaxkan kin tuwena he. na lye ki kta iyecetu xni, nakun nix niye exa niyecinka he-

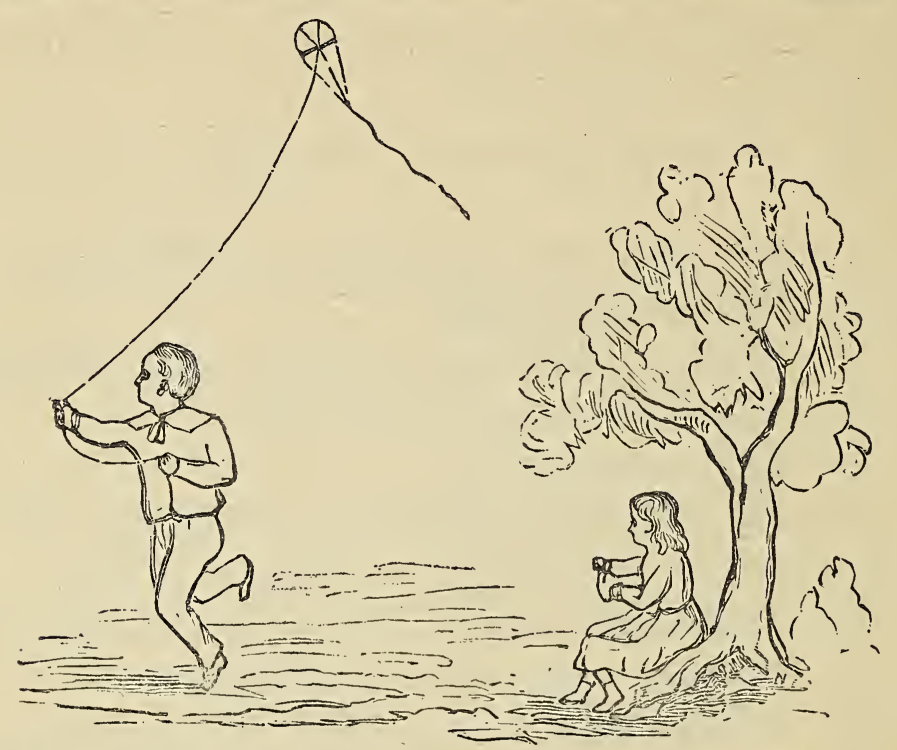

canon kirhan he woartani. Satan (wakanxica) iye hecon kta. kuwa, tka he anayagoptan kta iyececa xni, icin Wakantanka winawizi, qa wakagapi hekta Anpetu-wakan on ociciyake ciqon hececa, qaix taku tokeca Iye oxtan waxteyadaka qa wawokiye yaxkan kinhan, iyokipi xni.

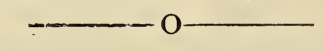

\section{WOONSPE XV.}

WOONSPE WICOIE-WAKAN.

Oekde-_-Nicaje wakandapi nunwe." st. Matt. 6;9.

W. Moahope iyamni kin eya wo.

A. Itaycay, Wakantanka nitawa, caje kin ituya ehin kte śni; tuwe iye Caje ituya eye cinhan, Itancan kin he woalitani ('ona un ecankin kte śni.

W. Woahope kin de ed taku itancanyan cajeyatapi kin he tukte e he?

A. Wakantanka Caje kin.

W. Toked Wakantanka Caje ituya unkeyapi kta uykokihipi he? 


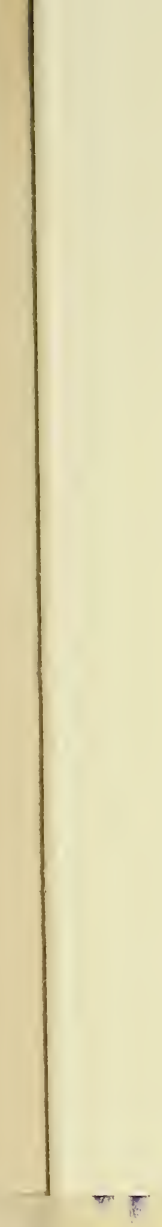

YUSNAPI. Yubdayapi 27 alsand iteowapi kin, he ardapsinyan iyeya owapi. 


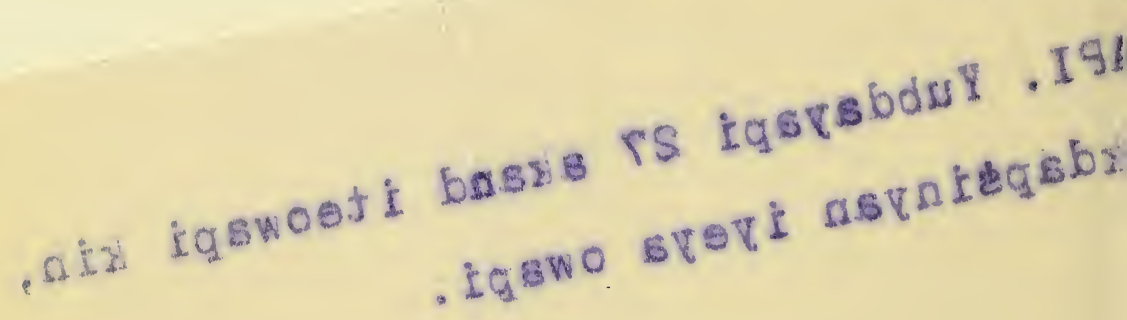


A. He takuundapi śni kiniyeced cajeunyatapi kin on.

W. Oekde kin ed toked Wakantanka Caje unkuwapi kta e onspeunkiyapi he?

A. Wakanuydapi kta onspeuıki api.

W. Wakandapi eyapi kin, he on toked kapi he?

A. Wakanyan yuhapi.

W. Woalitayi cona un, eyapi kin he on toked kapi he?

A. Iyaonpepica śni.

W. Woahope kin de kicaksapi kin, hena tona eepi he?

A. Tona Wakantanka iliad cajeyatapi, qais iyukcayśniyan taku eciyapi, qais heced Iye on taku eyapi kin, hena eepi.

W. Wakantanka tona wahtanipi kin toked wicakuwa kta he?

A. Kakiświcayin kta.

Iteowapi ded kagapi kin he Wakantanka Caje Wakan, 'Juda oyate toked iapi tawapi kin ed owapi tkat qon, he iteowapi kin ee, qa anpetu wi kin okxan iyojanjan yuke cin he iyeced wiceti ihe okxan kagapi, icin Wakantanka caje kin nina rcin wakan yedakax heced iyacinyan kagapi.

Hokxin-kagapi ni-

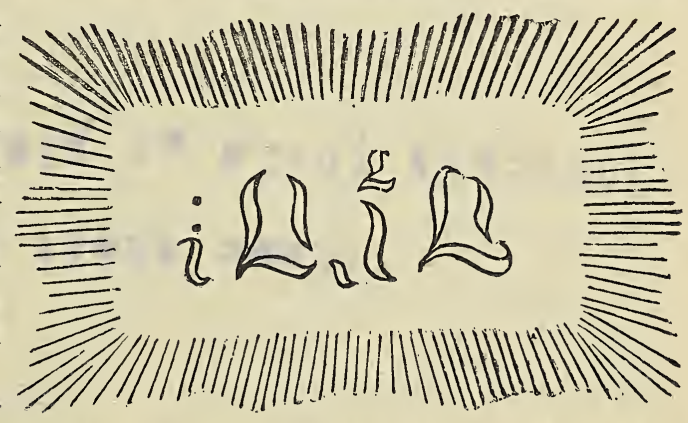
na waxte wanji, qaix wowapi owanyag waxte heca exa, duha canna ihun canopiye wan ed hetanyan oniciknaka ece, tehan waxte duha kta e heon etanhan. Nakun nisun qaix nitankxi wana ta tka.taku tawapi qon wanji duhe cinhan he iyotan nina tanyan awandakin kta naceca, icin he iye tawa tka qon on etanhan yuonihanpi kta iyececa sececa. Ho. Wakantanka Caje Wakan kin he Iye tawa, qa niye, cincaniye cin, taku 
maka akand un kin owasin isanpa he wakan yawapi kta iye. ceca kecanin kta, qa awandakin kta, qa Iye teridapi kin cante onijuna se, qa ho iwaxtena kicun, Caje kin he ehin kta iyececa.

Wakantanka tona iye Caje ituya eyapikin hena woartani cona un ecanwicakin kte xni. Icin, wayawawicakiyapi kin ekta woahope kin owasin rcin tanyan anayagoptan xni hantanbanx iyaonpepica xni niyawapi kte xni e he sdodyaye do. Wowasukiye wanjina hecena rcin yakicaksa hantan $\mathrm{x}$ iyanionpepica xni xni, unma kin owasin anayagoptan exa. Unkan woahope kin decena okaptapi Wankantanka toahope unma kin owasin anyagoptan exax Wakantanka iyanionpepica xni, qaix wakaxote $x$ ni, niyawa kta okihi kte xni, tka on kakixniyin kta, niye hecanon kin on icantenixica qa tuwe ixnana wicaxa iyaonpepica xni ni un qon, Itancan Jesus Christ Hee, e on nicicajuju kta iceyakiye xni ehantanhanx.

Iho, heon Itancan Tawncekiye kin on, Wakantanka Iye Caje wakanyada kta e oniciyin kta icekiya wo. Taku tona duhe cin ekna he jyotan wakanyan yuha wo; hecanon kinhan anpetu inicihan ed tokata Iye Caje Wakan kin itehu nitawa kin ed oniciwapi kta. He tawaniyan, qa tenirida kin tuwe. pike ceyax owasin he sdodyapi kta e heon etanhan.

\section{WOONSPE XVI.}

\section{WOONSPE WICOIE-ANPETU.}

Oekde.- "Itancan, anpetu kage cin he de anpetu kin, ee." Ps. 118;24.

W. Woahope itopa, onšpa tokaheya kin eya wo.

A. Aypetu Okihpapi kin wakanyan duhe kte cin he kiksuya wo. A ypetu śakpe htarani kta, qa taku ecanon kte cin owasin eced ecanon kta; tka aypetu iśakowin kin he Itancan Wakantanka nitawa aypetu okihpapi tawa kin hee. He ed wicohan takuna ecayon ktes̉ni; niye qaiś nicīks̉i, qaiś nicunks̉i, ookiye nitawa wica kin, qaiś ookiye nitawa winyal kin, waniyaypi nitawa, qaiś wicaśa tokeca nitatiyopa itimahed un kin. sa he?

W. He taku e unkakiktonjapi kta iyeuncecapi

A. Wakaytanka taajpetu wakayyan unyuhapi kta. 
W. Hena tuwepi e Wakantanka taanpetu wakayyan yuhapi kta kiksuye wicaśipi he?

A. Wicaatkuku qa wicahunkupi kin.

W Nakuy tuwe he?

A. Wakanhejapi kin.

W. Qa nakul tuwe he?

A. Wowaśi unpi kin.

W. Qa nakuy tuwe tokeca he?

A. Ho, icimani ed unhipi kin hena.

W. Wakantajka taajpetu kin icuıhay wamakas̉kan asnikiyapi kta iyowinwicunkiyapi kta iyececa he?

A. Ho, woahope kin "waniyanpi nitawa" eya.

W. Toked on Wakantaıka taanpetu kin wakaıyan duha kta oyakihi he?

A. Wakan-cekiyapi ekta owapa kin he, qa toked owakihi kiı Wakantanka qa Malipiya on onspemiçiciye cin on.

W. Anpetu Wakan kiń icunhan yaśkatin kta iyececa he?

A. Hiya.

Wowapi wan ed takuna owapi xni, tka ataya wowapi ska ece ojuna, heca tohinni wandaka he? Anpetu otoiyohi takuxnixni akipapi kin hena ed owapi kta on wowapi hececa

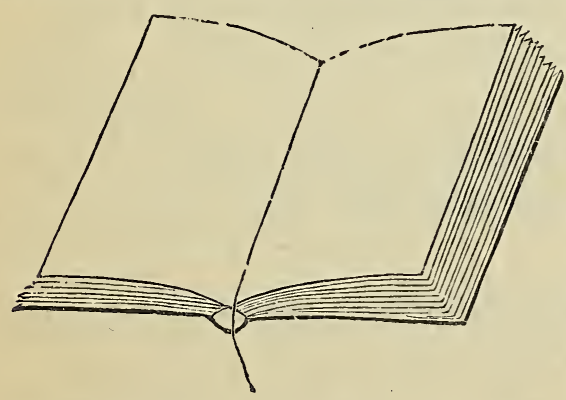
tuktekted yuhapi. Heca wanji nicupi kinhan iyonicipi kta he? Okinni wanji duha exa ed taku oyawa kta oyakihi kte xni, kecanni naceca ecanmi. Tka Wakantanka niye qa nakun wicaxa owasin, wowapi ed takuna owapi xni otoiyohi wicaqu, qa anpetu iyohi oyubdaye ska kin akand takuku oyawa ece. Oyubdaye kin hena otoiyohi anpetu heca, unkan wowapi unkitawapi kin ed oyubdaye tonakeca unkiciyukanpi kinhan tohinni sdodunkiyapi xni, exa xakowinwin ece ptaptaya eknakapi. Heced de anpetu kin, Wakantanka taanpetu kin hee, he oparte wanji 
ed wowapi oyubdaye tokaheya kin hee, unkan he akand ka ked oyawa nin ecanmi; "De anpetu kin wakan-cekiyapi qa Anpetu-wakan onspewicakiyapi kin ekfa wai. Taku waxte onspemiciciya, qa toked oqoye xni mikduha kta, qa de anpetu kin Wakantanka taanpetu heca e kiksuya waun kta wakuwa." Qa oyubdaye unma xakpe ska kin akand, hanrana qa rtayetu iyohi Wakantanka iceyakiya, heced oyawa nin ecaumi; qa yaxkate cin he qa wowaxi ecanon kin qa wowapi onspeniciciye cin koya on Wakantanka iyokipiyayin kta yakuwa; qa ohini waonnixida qa wowicake ece eya nikduha, qa niyate qa nihun anawicayakigoptan kta yakuwa. Hecekced taku oyawa ece kinhan Wakantanka iyokipi kta; tka oran oyakapi nitawa xica kinhan, anpetu iyohi xikxicaya oyaran, qa oyubdaye owanyagwaxte ska tka qon hena wicoran owotanna xni on axabyaye cinhan, hececa qeyax nakun Wakan. tanka iyuhawanyag un, unkan icantenixica ehantanhanx, Wanikiya waxte kin on, nicicajuju kta, exa takomni Anpetu-wakan oko kin de wowapi oyubdaye tawa kin piya oyawa kta e tohinni rcin nicupi kte xni.

Ho, heon etanhan anpetu kin de etanhan heced econ aya wo, qa Wakantanka wowapi wan niye nitawa kta e on nicu kin, ed taku waxtexte ece oyawa kta e oniciyin kta icekiya wo. Wowapi kin de caje kin he nahanrca ociciyake xniAnpetu, heced eciyapi.

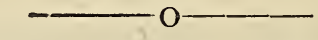

\section{WOONSPE XVII.}

WOONSPE WICOIE-OKIRPA.

Oekcle.- "Anpetu ixakowin kin he Wakantanka okirpa, taku kage cin owasin etanhan." Heb. 4;4

W. Woahope itopa onśpa inoppa kin eya wo.

A. Aypetu śakpe ed Itaycay kin mahipiya maka irakna kağ́a, miniowayca kin, qa taku oknayay un kin owasin, qa aypetu is̉akowin kin ed okihpa: heon etayhay Itaycan kin aypetu iśakowin kin yuwaśte qa yuwakay.

W. Wakaytanka malipiya qa maka kin tohayyay kah uy he?

A. Aypetu śakpe.

W. Wakantanka anpetu tokahesa kin ed taku kaġa he? 
A. Iyojayjay. ğa he?

W. Wakaytanka anpetu inoypa kin ed taku ka-

A. Mahipiya to kin.

W. Anpetu iyamni kỉ ed taku kağa he?

A. Maka qa miniowalyca kin.

W. Aıpetu itopa kin ed taku kağa he?

A. Aypetu wi, hayhepi wi, qa wicallipi kiø.

W. Wakantanka anpetu izaptan kin ed taku kagia he?

A. Zitkana qa hogay.

W. W akantanka anpetu is̉akpe kin ed taku kağa he?

A. Wamakas̉kan qa wicøša.

W. Wakantanka anpetu iśakowin kin ed taku to$\mathrm{k}(\mathrm{m}) \mathrm{h}$ ?

A. Wicoliay tawa kin owasin etanhay okilipa.

W. Wakantanka anpetu tukte wajji yawaśte he?

A. Is̉akowin kin, anpetu okilipapi kin hee.

W. He tokeca e Christian uypi kin anpetu is̉akowin kin heekiya anpetu tokakeya kin, Anpetu-wakan ecijapi kin he, wakanyay yuhapi ece e he?

A. Wahośije Wakaypi kị iye heconpi kin heon etanhay:

W. He tokeca e iye heced ecoljpi he?

A. Christ Aypetu-wakan kin ed wiconte etanhan kini kin heon etal hay.

W. Christ Iye heced econwicaši unkecinpi he?

A. Ho, heced unkecinpi.

Tuwe maka kin 'saga he? Ho, maka owanyag waxte kin de, qa can, qa warca kin, qa wamakaxkan, qaix ate qa ina qa unkitakodakupi tewicunridapi kin hena wanwicundakapi canna; qaix tehan wankan euntonwanpi qa anpetu wi wiyakpa kin he, qaix hanhepi canna wicanrpi iyeryega kin hena 
unkabdezapi kinhanhan, tuwa taku kin dena owasin kagahuwo? unkecinpi kta iyececa. IIakantanka ixnana tanka kin, Iye hena kaga. Iye maka kin nape cokaya okna yuha un se, he iyececa. Hekta Anpetu-wakan onspeniciyapi qonhan, ed wowaxi ecanon kta qa yaxkat in kta e on Anpetu-wakan oko wanjina ed anpetu tona niciyukan onspeniciyapi he? Unkan anpetu wanji wakanyan duha kta iyececa kin he tukte e he? Wakantanka Iye rca he iyeced oran qon eciyatanhan on he onspeniciya, icin maka kage conhand wowaxi econ--an petu tonarea he? Unkan anpetu wanji ed okirpa qon he tukte e he? Ho, he iyeced cinca kin Iye wowaxi econ qa woasnikiye tawa kin ed kici akiyecedya $\mathrm{xk}$ a $\mathrm{np}$ i kta cinwicakiya.

Tohinni wakanheja wanjigji can xoka wan, tukted mnaja ocinxica wan wicakte kta iyececa, can wekna

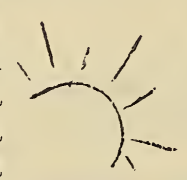

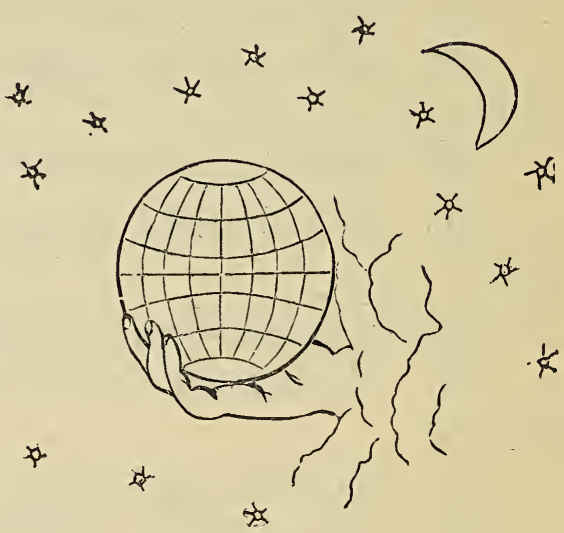

$x$ okokipeya o mani pi tka qon. Tka wicaxayatapi waxte wan wojupi tawa ikiyena he cin hed conkaxke itimahed awicahi, unkan woope wanji owicakiyake cin he ahopapi kinhan, iye ti waxte kin wiyohiyanpata conkaxke tiyopa kin etanhan taninyan he cin, hed okna iye kici ounyanpi kta e tokata anpetu iwicakicihan kewicakiya. Woahope qon he dee: Anpetu iyohi wowaxi qa woxkate kduxtanpi qonhanhand. wojupi kin ed tuktedtanhan wieaxayatapi ti wanyakapi okihipi kte cin hed mazaxiranxkan oape wanji owanjina unpi, qa wicaxayatapi kin he qa tipi wan okna tokata ounyanpi kte cin he nakun on woyake naron yankapi kta. Unkan owasin woope wan heced ahopa terike $\mathrm{xai}$ qon he iyuxkinyan anagoptanpi kecannipi he? Hehehe! Hunr wowaxi econ ayuxtanpi kta okihipi xni kecinpi. Qa ake tokecapi wojupi kin ed oyanke ed asnikiyapi kta sanpa oiyokipi iyeyapi; qa hunr mnaja yukan wicadapi xni keyapi, unkan can wekna hedtu rca kayex ekta kikdapi. Hececa exa wakanheja qon wanjigji wicaxayatapi woope tawa qon anagoptanpi, unkan he okna asnikiyapi kin on sanpa waxakayena wowaxi econ okihipi heced iyeyapi. Hena wicaxayatapi qon ixnana takodakupi waxte rca hee ce eya ijehan awacinpi, qa 
iye wicakico canna iyuxkinpi, qa ite ira ikdus ed yapi ece. Qeyax mnaja qon tona can wekna kikdapi qon hena owicayuspa, qa nakun wojupi kin conkaxke paoksa unkan tima hiyuiciye, ca tona wicaxiyatapi ti qon itehanyan asnikiya yankapi qon hena ontonwicaya keyapi. Wicaxayatapı qon he Wakantanka ee. Iye wojupi tawa kin, Obodakiciye Wakan kin hee, e ed yaun, unkan mnaja wan maka kin ed "tuwe yapotin kte cin ode" omani un kin, he wakanxica ee. Wakantanka wowaxi ecanon kta qa yaxiratin kta e, on anpetu xakpe nicu; tka anpetu ixakowin kin heehand wakancekiyapi, tukted lye qa Marpiya, wicaxayatapi tipi tawa kin tokata ounyayin kte cin hee, hena on onspeniciyapi kin hed de kta cin.

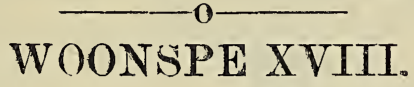

WOONSPE WICOIE-WOYUONIHAN.

Oekde.-."Wakanheja kin, nihunkalepi kin taku owasin anawicakigoptan po, he Itancan kin iyokipi." Kolosse, 3:20.

W. Woahope izaptay kin eya wo.

A. Niyate qa nihun wicakduonihay wo; heced Wakantanka nitawa Itancan kin, makoce nicu kin ed, nituanpetu kin hayskin kta.

W. Wuahopo kin de iyowajapi kin hena tuwepi he?

A. Wakanheja.

W. Wicaduouihay kta iyececa kin hena tuwepi he?

A. Ate qa ina.

W. Toked hena wicaduonihay kta oyakihi he?

A. Hena anawicawakiğoptan kta, qa tewicawahida, qa owicawakiyin kta iyececa.

W. Wowapi Wakain kin ed woahope kin de toked cijeyatapi he?

A. "Woahope on taku wahoyapi."

W. Taku wahoyapi kin he tukte e he?

A. Wiconi hay-ka.

W. He tuwe e hered wahowicaya he?

A. Wakantanka Iye heced wahowicava.

W. Nirate qa nihuy wicaduonihay kinhan he tuwe $\theta$ iyokipi he? 
A, Itarjeay $\mathrm{k}^{\circ} \mathrm{r}$.

Woiwahoye eyapi kin he on toked kapi kin sdodyaya he? Mazaxkánxkan oape ciscina zaptan hehanyan owanjina narke cinhan, miniruha owapi wanji wicite owapi waxtexte ojuna cieu kta eciciya ehantanhanx, he woiwahoye heca kta he? Wakantanka taku wanji wahoniyan-

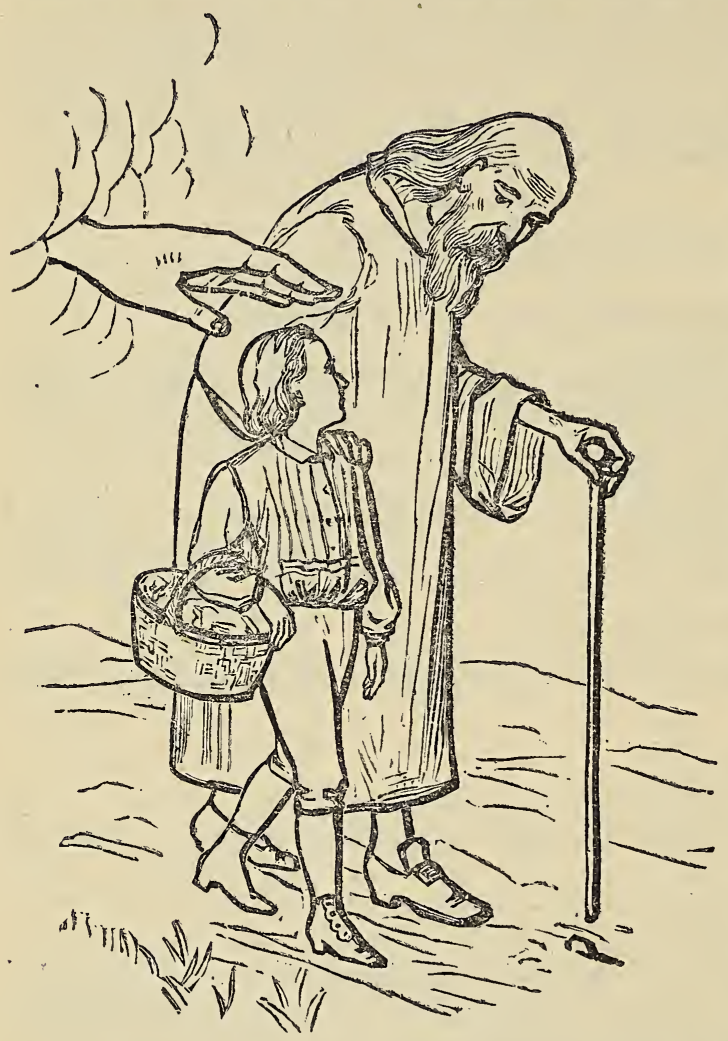
he taku he? Niyate qa nihun anawicayaki g optan xni ehantanx, taku kin he nicupi kta iyeniceea he? Wakantanka taku wahowicaya kinhan ohinni eced ec a w i c a kicon he? Ho, ll akantanka taku unkekiyapi kmhan hena owasin wowicake rea ee sdodunyanpi kta iyececa. Hokxina nina ciqana wan tka atkuku ytonihan kin on nikiyapi qon he, ito, on ociciyakin kta. Maya wankantuya wan icikca xkata un, unkan urma eciyatanhan maza canku ocauku kin ikjyena wa $\mathrm{na}$. Atkuku kin hetanhan ikyena ceartonpi wan awanyag xipi, unkan cinca okokipeya najin wankdaka exa ekta yin kta okihi xni; icin, ceartonpi awanyag un qon he ayuxtan qa tokan iyaye cinhan okinni wicaxa ota ed wicaktepi kta naceca. Heced atkuku kin panyanhan hoyekiya qa "Cinkx, makata erpeiciya kud wanka wo," eciya. Unkan hokxina ciscina qon, "He toka e kud wanka mayaxi he?" eya ayupta ecanni he? Hiya, atkuku kin iye hecon xi dakax heceknana kud makata wanka erpeiciya, unkan icand ikwuhana mazacanku canpakmi ota icikoyagyapi heca wan orankoya ed ahiyaya exa ni kdicu; tka atkuku toked econxi qon he wancakna anakigoptan xni unkanx ed ta tka.

Nakun hokxina wanji, kanipe xni hanska, exa. He ohinni 
Atkuku qa Hunku wicakduonihan tka qon. Hokxina kin $\mathrm{He}$ Wanikiya waxte unkitawapi kin, Jesus, Hee. Iye toked un qon nix eya iyeced nikduha kta e yakuwa kta Wakantanka cin. Hecanon kinhan Itancan woyawaxte tawa kin anihin kta. unkan wawicaqupi waxte rea, Wakantanka Iye cinca kin owasin wicaqu kin, wiconi Narpiya Makoce kin ekta, wiconi owihanke wanica kin he kapi, he niye nakun nitawa kta.

\section{WOUNSPE XIX.}

WONNSPE WICOIE-WOWARTHDAXNI.

Oekde.--"Tuwe hunkawanjitku xicekidake cin he tidwicakte kin heca." I John, $3 ; 15$.

W. Woahope is̉akpe kin eya wo.

A. Tidwicaktepi ecanon kte s̉ni.

W. Wakantanka woahope tawa kin unkicaksapi hecinhay he sdodyin kta cin kinhay, tukted etonway he?

A. Unkicantepi kin mahed.

W. Cayteoyuze kin tukte wanji tidwicaktepi iyececa he?

A. Wowahtedas̉i.

W. He tokeca e wow ahted as̉ni canteoyuzekin tidwicaktepi iyececa he?

A. Tuwe wahteundapišni kinhạ, okinni he kte ancinpi kta naceca heon etanhan.

W. Canteoruze tukte wayji unki santepi kin ed engkiciknakapi kta e Wakantanka iceugkiyapi kta iyececa he?

A. Wacantkivapi.

W. Wakayheja tokera cantewicayakiye cinhay kiugniwicayakiy in kta yacin kta he?

A. Hiya.

W. Toked ecawicayecon kta yakuwa kta he?

A. Cantewaštewicawayin kta.

W. Tidwicaktepi ivaogpepicas̉niyan taku unktepi kta unkokihipi he?

A. Ho, woyute unyampi kta e on wamakaśkan anktepi kta iyowinugkiyapi. 
W. Eśa waoநsiyada śni, qa otuyacin yazaĐwicayayin kta iyonicipi hecighan toketu he?

A. Hecamon kighan woahope kin de wakicaksa heca kta.

Wayawa de cinhan, qa hokxina wan can adetka wapepeka. ojuna yuha canku okna ayakipa, unkan he on ite qaix nape ed anipa kinhan, he canteniciya qaix canteniwaxte kta cin kecanyakin kta he? Hokxina kin he taku canteoyuze okna xkan ecanyakin kta he? Wicaxa wan, nape okna mina yuha, qa canzeka se iteoyuze xica, wicaxa tokeca ektakiya inyang ya wandake cinhan, he cante kin ekta wowartedaxni yukan tanyan sdodyayin kta, ci? Wakantanka wa pe peka qa mina uncantepi mahed yuke cin hena wanyaka okihi, unnapepi kin okna taninyan he xni itokab. Wakanheja tokeca

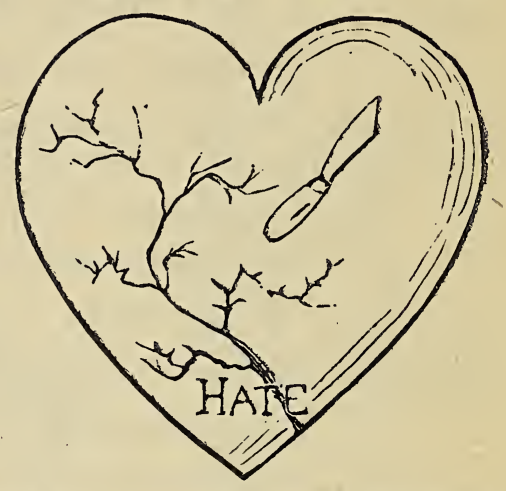
wicadujipin kta, qa awicayapin kta, qa cantexidwicayayin kta iyonicipi, qa xunka qaix ikmuxunka yazanwicayayin kta iyonicipi ehantanhanx, nicante mahed wapepeka seececa wowartedaxni niyukan.

Wicaxayatapi xica wan, Nero eciyapi qon, qa wicaxa tanka icaga qonhand wicaxa qa winyan ko wicakte kta e on mina ota wiyeya kiknake ciqon, he hokxina qonhand waimagagaiciyin kta e on honagina wicakte kta iyokipi tka qon. Unkicantepi kin mahed wowartedaxni ciscina toka hinnape cin he hed yankin kte xni e itonunkicipapi kta iyececa, okinni tanka icaga unkan wapepeka qa mina cante ojunaunkivapi kta naceca heon. Tuwe tokeca wanyaka, qaix wanyakapi xni exa, Wakantanka iye he ekta etonwin kta, unkan tidwicaktepi iyaunqonpapi kta. Nicante kin wocantekiye on ojunaniyin kta e Wakantanka icekiya wo. Nitanokxan unpi kin owasin cantewaxtewicayayin kta kuwa wo. Heced ecanon kinhan woahope ixakpe kin he tanyan ahoyapin kta.

\section{WOONSPE XIX.}

WOONSPE WICOIE-WAKAXOTEXNI. $51 ; 15$.

Oekde.- "Wakantanka, cante ska micaga ye." PsaIm

W. Woahope išakowin kin eya wo. 
A. Wawicihaliapi ecanon kte śni.

W. Oekde kin ed wicoie kin tukte wanji on wakaśoteśni kapi he?

A. Ska.

W. Woahope kin de taku onspeniciva he?

A. Micanłe kin woawacin sica on aśape śni mikduha kta.

W. Tokel he wakaśoteśni nikduha kta oyakihi he?

A. Wicoie śikśica wannag̉optanyan nawahor śni, qa wakanheja śkśica ob onyware śui kin on.

W. Nakul taku ecrnon kta ivececa he?

A. Mayu-ka·kta e Wakanłanka icewakiyin kta iyececa. he?

W. Wakantalka cante skaskapi kin on toked eya

A. Mena wicayawaśtepi ce, eya.

W. Wowiyuskin tukte wanji tawayapi kta he?

A. Wak:intanka wayyakapi kta.

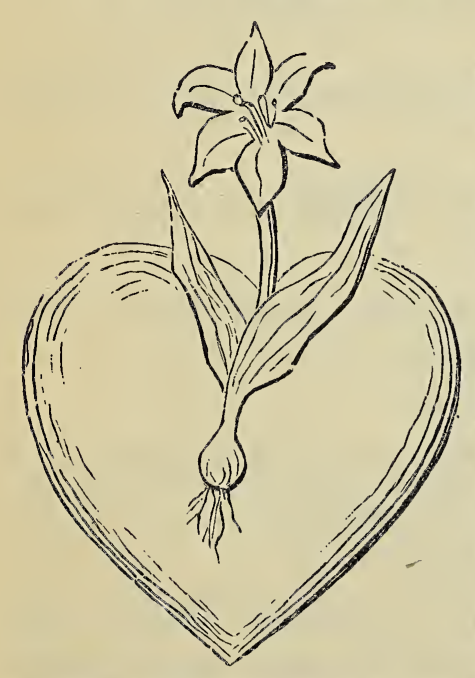

Mnarcarca ska heca tohinni wojupi wan ed icaga wandaka he? He toked wa iyecad ska rea, qa waxtemna qon yeksuya he?

Nicante mahed wojupi ciscina wanji yukan, unkan wanarcarca toktokeca hed icage cin ekna Wa kantanka warca wanji ska qa owanyagwaxte, mnarcarca iyeced wakaxotexni qa waxtemna, icarkiya. Toka nitonpi ehantanhan hed un, unkan hokxiyoqopa yaun qon ehand wa kin isanpa ska; qa nahanrca dehanyan ska exa he ehand toked ninar ska qon dehand iyeced wakaxotexni xni. Wanarcarca kin de caje kin ociciyakin kta yacin he? Woiyaonpepicaxni, heced eciyapi. Warca wan heced owanyagwaxte kin he nina tanyan awanyakdakin kta, ci? He toked yakduxicin kta iyececa kin he ito, iwaktayaked oci- 
ciyakin kta. Tohanhanxna woyakapi xica, qaix wicoie xikxica exa, nihun waxte hena sam oyakidakin kta tawatedyayin kte xui tka kin heca, waanagoptanyan naron nanke cinhan, iyena marpiya xapa wan warca ska kin de iwankab ahi wanka, qa tohanhanxna taku xica ehe cinhan, qaix woawacin xisa iyowinniciye cinhan, iyena woaxape wanji warca ska owan. yagwaxte kin de ed atanin. Warca waxtemna kin de tanyan awanyakdakin kta yakuwa xni kinhan, sanpa qa sanpa axab. yapi ayin kta, unkan unhanketa xnixya hinrpayin kta.

Hancokaya wa kin he anpa ed wanyakapi kin, toked owanyagwaxte hinhan unkanhe sdodyaya. Nina,ska qa wakaxotexni rca onetanhan anpetu wi kin aiyojanjan kinhan ixtaiyonixnija. Exa anpetu wanji qaix nonpa unkan wana xapa aya. Nina amanipi kin on naxabyapi, qa maka bobdu qaix taku toktokeca ota on yuxicapi. Nicante kin he wa kin de iyececa. He ska niciyuha kta e Wakantanka iceyakiyin kta iyececa. Ito, Wowapi Wakan kin etanhan wocrkiye ciscina wan ociciyakin kła unkan he eyakiyin kta. "Niye mayadujaja kinhan, wa kin isanpa maska kta."

\section{WUONSPE XXI.}

WOONSPE WICOIE-CANKU.

Oekde.-"O Itancan, nitacanku kin onspemakiya ye." Ps. $85 ; 11$.

W. Woahope iśakdog̀ay kin eya wo.

A. Wamayanon kte śni.

W. Woahope kin re taku iyoniciśni kin he tukte e he?

A. Taku mitawa śni eś ı iwacu kte cin, heiyomakiśni kin ee.

W. Taku way nina ciscina eśa icupi kị he śica he?

A. Ho, he wamanojpi heca.

W. Wakanheju wal woahope kin de tayyal ahope ciuhay toked he cajeyatapi ece e he?

A. He olial owotanna eyapi.

W. Iye iyowinuyyanpi śni eśa tuwe tokeca caywopiye-yusdutapi, quiś calkokana tawapi kin mahed eujtonwaypi kta iyececa he? 
A. Hiya, woahope kin de wakicaksin kta e he on yusam:iyıpi kta naceca.

W. Woahope kin de huha awanikdag unśpi kin hena tukte e he?

A. Unnapepi.

W. Unnapepi kin woahope iśakdoğan kin ahopapi kta e taku econ okihipi he?

A. Wowasi waśte qaiś wawokị eka.

Wowapi Wakan kin ed Wakantanka deced onspeunkiyapi; wicaxa oran owotanna kin he tacanku kin ojanjan iyacinpi, qa

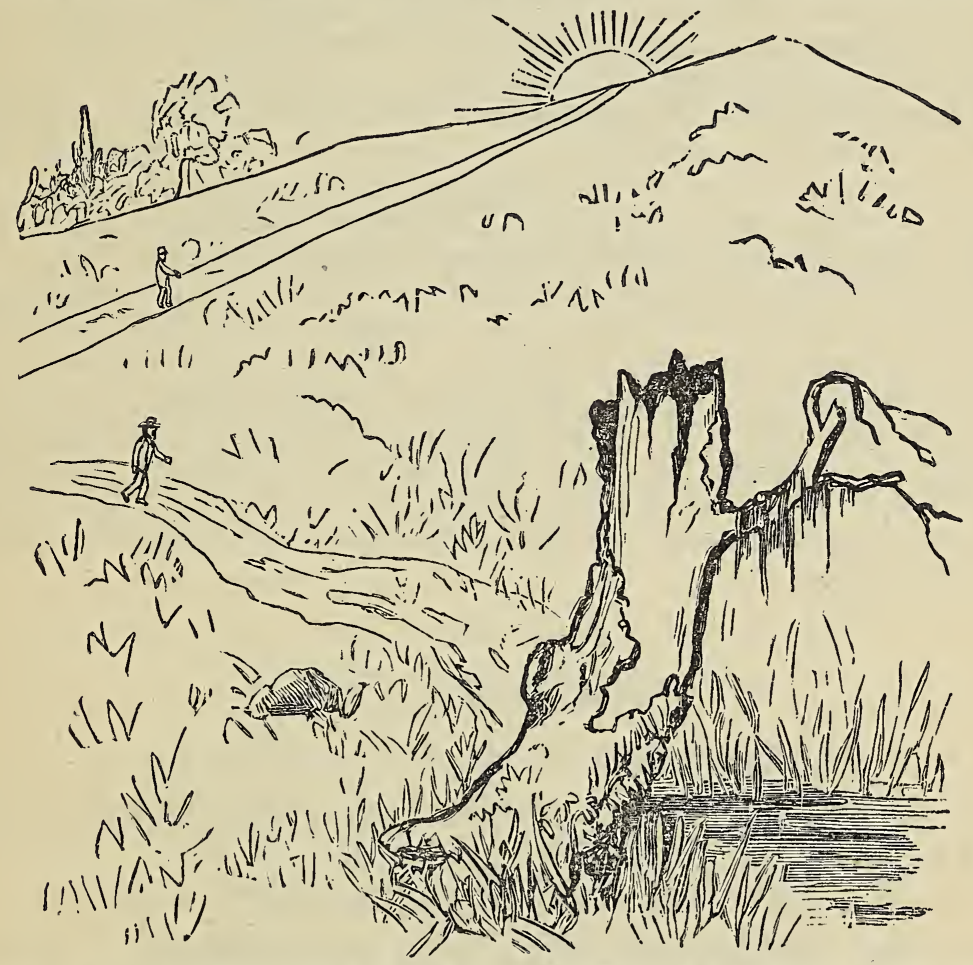

tuwa owotannayan oran kta kuwa kinhan he tukted ojanjan kin tacanku iyokicijanjan kta e ekta iyuxkinyan ya ece, taku econ kin tanin kta e, qa Wakantanka hecetukicidakin kta heon. 'I'ka wamaronsa kin he hanhepi canna narmana waode omani ececa e słodunyanpi, icin wana wi mahed iyaya, qa wanyakapi kta cin xni kin heon. Heon etanhan canku tawa kin oiyokpaza kin etkiya, qa anpetu wi kin itehanyan wanka. Tka wicaxa oran owotana kta kuwa kin he wanyakapi exa 
ixtece xni, qa anpetu wi kin ektakiya mani, icin he waxtedaka dakax, qa toran kin owasin Wakantanka hena sdodkiciyin kta, unkan sanpa waxłe kta e okiyin kta cin kin heon hececa.

Wowapi wan ed wowapi nape on kagapi onspeniciciye cin ed oyubdaye wanji toker oyakihi tanye rcin akand oyawa kinhan, niyate qa nihun qaix waonspekiye nitawa hee exa toked oyakihi tanyan yakaga wanyakapi kta e heon hena wanji kici ojanjankdepi ekta kduha de kta e cantewaxteya wicayadakta. Tka tanyan yakagin kta yakuwa xni hantanhanx, unkan oyubdaye kin he xicaya owapi qa minisapa axbuyapi yukan kinhan, wowapi kin canwopiye-yusdutapi wan mahed tukted ojanjan wanica qaix wanya api kte xni, hed mahed oyaknaka yacin kta.

Ho, nix eya dehand canku owotanna okna, qa anpetu wi ojanjan kin etkiya, qa Wakantanka iyokipiya omani yaun, qaix oiyokpaza etkiya canku yukxankxan okna idada, qa takuxnixni nitawa xui exa iyacusa, qa nakun wicoran owotanna xni on Wakantanka iyopeniyin kta heca ecanonsa. Anpetu wi wiyakpa kin on atayena ekta eyatonwin kta oyakihi xni; exa can qaix inyan ciscina wanji iyacu qa niixta ikiyena rcin duze cinhan wana anpetu wi kin ecaca wandaka oyakihi kte xni. He iyeced taku xica ecanon kinhan cisciscina exa hena otoiyohi Wakantanka wandakin kta e okihixniniya. He marpiyata niyate tenirida kin ee, qa iye itokan yaun kinhan tohin. ni canteniwaxte oyakihi kte xni. Kiksuya wo; "Wakanheja exa oranye cin etanhan ikdutanin"-Wakantanka iye heya. Ninape cisciscina kin hena wankdaka wo, qa wicanap taku waxte ece econsa, qa wawokiyeka, qa nitanokxan unpi kin owasin ekta waonxida, heca yakagin kta kuwa wo, qa hehan Wakantanka canku ojanjan tawa kin okna yusaniyin kta e icekiya wo.

\section{WOONSPE XXII.}

WOONSPE WICOIE-WAAWANYAKA.

Oekde.- "O Itancan, mii kin ed waawanyaka ekde ye miiha tiyopa kin awanyaka ye." Ps. 141;3.

W. Woahope inapcinwanka kin eya wo?

A. Nikiyena-ti kin, ituya ayaie kte śni.

W. Woahope kin de taku onspeniciye cin he tukte e he?

A. Wowicake ece iwayin kta.

W. Taku way ed cajeyatapi qa he econ śni uy unśipi kin e tukte e he? 
A. Wicaśa tokeca owewicakiwakaypi.

W. Taku wayji wicakapi qais̉ wicakapi s̀ji hecinhay sdoduyyaypi śni ehaytans', hehay toketu kta he?

A. Hececa kinhay saypa unkoyakapi kta iyececa s̉ni.

W. Tokaheya hivin owewakan qon he tuwe he?

A. Wakayśica.

W. Wowapi Wakay kin ed nakuy toked eciyapi he?

A. Owewakankanpi atkuku.

W. Waoıśida śni, qais̉ canzeka, qais̉ owewakankaypi wicoie iyae cinhay, he ol woahope tukte wayji yakicaksa he?

A. Woahope inapciıwanka.

W. Taku tanyan awanyakdakin kta iyecece cin he tukte e he?

\section{A. Miceji.}

Ded owapi kin hena taku he? Ho, hena tiyopa heca, unkan de ancetu kin tiyopa, inyan terika ocaje wanji xa rea kin he iyeced xaxa kin on nina owanyag waxte, heca on ociciyakin

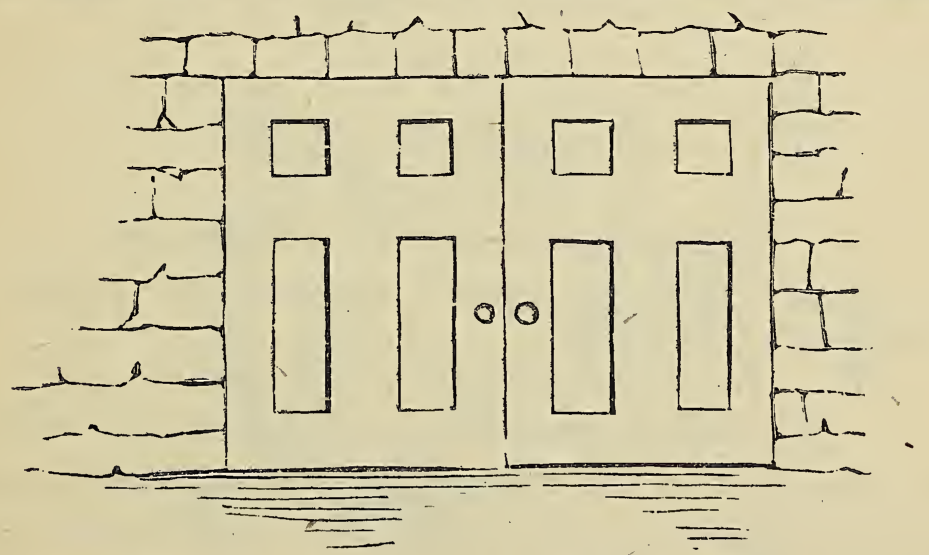

kta wacin. Tiyopa kin hena yurdokapi terike xni ececa (tohini inatake qaix iyuxdoke ed atanin xni), unkan yurdog iyaye cannahan wowaxi cisciscina ocibdar najinhan najin qa owasin skaska ikduzapi, itimahed otaninpi. Qa tuwe wanji 
tipi ciscina kin he oti, unkan he tohand ikdutanin kta cin kinhan wowaxi skaska ocibdar najinpi qon icunonb iyayapi, unkan winyan ciscina xa ikduza, qa tiyopa ed okxan wakid najin wandakin kta. Winyan ciscina xa ikduza kin de nina ie kta iyokipi, unkan tuktekted zitkana hotonpi kin iyect ho onaron waxte on wicoie nina oiyokipi ie sa. Exa ijehan tiyopa ciscina kin inarni yurdogiyaya wanbdaka, unkan wowaxi kin icunonpa ipsid erpeiciyapi, winyan ciscina xa ikduze cin nina canksiye rcin heyin kta e heon; "Etanhan tokeca-he nina taku warte xni, qa ni waun kin hehanyan icinonpa taku ewakiyin kte xni." Unkan hececa cannahan tiyopa ciscina kin ix eva canzeka se orankoya ake eced iyeiciyapi, qa owanyag waxtexni. Winyan sa ilsduze cin caje ociciyake cinhan, okinni tiyopa kin dena taku tiyopa kin isdodyayin kta naceca. He caje kin Miss Ceii ee, qa niye i ciscina nitawa kin hed itimahed iyotankehan yanka, unkan iye itokab tivopa xaxa owanyag waxtexte kin eced iyeya han.

Owicakaxke tipi tiyopa kin ed tiyob inatake suta rca yukan, wicaxa xikxica tankad hiyu okihipi kte xni e heon etanhan. Tka tiyopa ciscina kin dena tiyob inatake qu iyuxdoke wanica, heced hena etanhan wicoie wicakrpi qa oiyolipi hececa ecena hetanhan hinapin kta $e$ on toked awanunkdakapi kta he? Oekde kin ed wocekiye kin he unkuupi kta."O Itancan, mii kin ed waawanyaka ekde ye, miiha tiyopa kin awanyaka ye," unkan hehan wicois xikxica takuna hetanhan hinapin kte xni e on Wakantanka iha-tiyopa unkitawapi kin awanunkici yakapi kta e awicakehan uncinpi kin he kiksuya unkonpi kta.

\section{WOONSPE XXIII.}

WOONSPE WICOIE-WOCANTOKPANT.

Oekcle.-."O Wakantanka, imayukcan, qa micante kin sdodya ye." Ps. $139 ; 23$.

W. Woahope iwikcemna kin eya wo.

A. Nikiyena-ti kin, ti kin yakon kte s̉ni tawicu, qa taokiye wica, qa taokiye wilyan. takayka tawa, soljsolna tawa, qa taku tawa kin wanjina kaśa yakon kte śni.

W. Wakonpi eyapi kin he on toked kapi he?

A. Cantokpanipi. 
W. Taku cantounkpanipi kta iyececa śni kin hena tona ee he?

A. Taku kaśa tuwe tokeca tawa kijhay.

W. Tuwe tokeca taku tawapi cantounkpanipi kinhan, ocib taku econkorjpi kta e yus unkayapi kta he?

A. Tuwe tokeca taku tawapi kin mauynoypi kta.

W. Hecolkonpi kinhan, on woahope unkicaksapi kte cin hena tona ee he?

A. Iwikcemna qa iśakdoğan ki .

W. Wocantokpani unkitawapi kin hena tukte tayhay hinapa he?

A. Unkicantepi kin etanhan.

W. Wocantokpani iyotan waštes̉te unkokihipi kin hena taku he?

A. Wocekiye.

Zitkana cikciqapina ota ded owapi: hunr wicacante wan etanhan kinyan hinapapi, qa hunr cante kin mahed horpi wan okna yankapi.
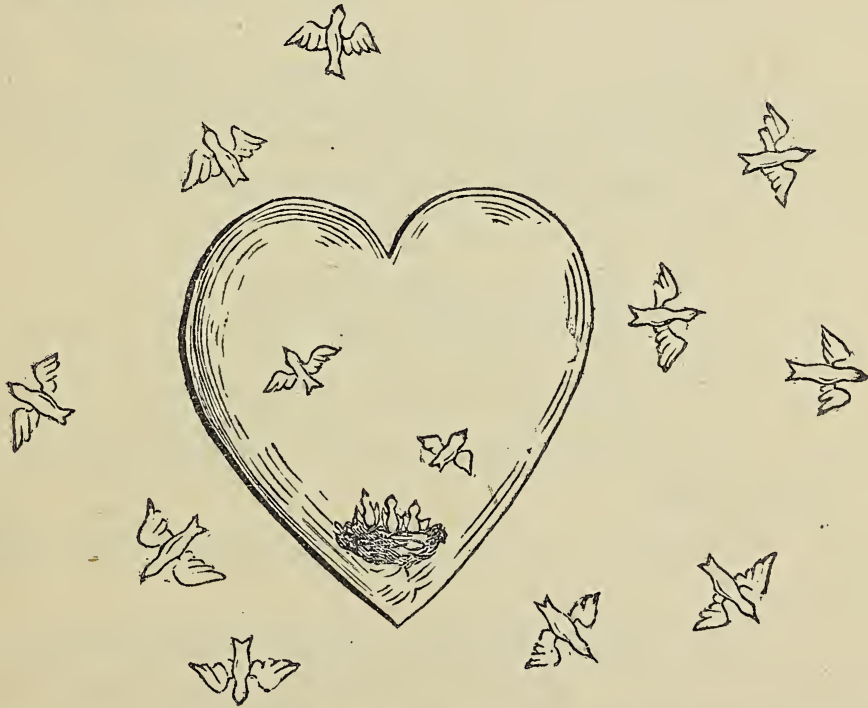

Zitkana cikciqaapina kin dena wocantokpani hecapi, qa ni an. 
te mahed wanbdakin kta owakihi ehantanx, hed nakun heca iyewayin kta e tanyan sdodwaya. Nicante kin okna wocantokpani yuke cin hena owasin wanyaka okihi kin He Tuwe he? Ho, Wakantanka. lye heced okihi, Oekde wan nakaha ehe cin he Wakantanka nicante kin mahedtuye rein etonwe, ca wocantokpani hed wahorpi kagapi kin hena taku wocantokpani hecinhan he abdezin kta e on icekiyapi. Oekde kin de wocekiye heca, unkan wicoie kin hena iyae cin icunhan wicayake rca hantanx he wocantokpani iyotan waxte nitawa kin heca wanji ee kta. He tokeca e Wakantanka unkicantepi kin hena sdodunkiciciyapi kta $\Theta$ iceunkiyapi he? Icin, Iye woxice unkitawapi kin hena unkipazopi kta, unkan sanpa unkiwaxtepi kta e ounkiyapi kta heon etanhan. Tohand niwaxte kta yacin canahan, he nakun zitkana wan Marpiya etkiya wankand kinyan iyaye cin he iyececa. Wocantokpani nitawa kin hunr okxan kinyan iyayapi exa takuna xica econpi xni; anpetu owaxtecakin kta yacin, qaix oiyokipiya tokiya icimani nin kta e cantiheyaye cinhan hena hececa.

Tohand warapixkanye wanji wakanheja tokeca tawa tka niye duha kta yacin kinhan, wocantokpani kin he xicaya wakonpi heca, unkan he zitkana wan kudkiya kinyan iyaye cin be iyececa. Tohand nihunkakepi qaix waonspeniciyapi kin ix toked econ nixipi kin hee xni tka ee niye nitawacin okna yaxkan kta yacin kinhan, iho, ake zitkana wan makatakiya kud hiyuiciya hececa. Unkan wocantokpani hececa kin xica, Wakantanka woahope tawa kin kicakseunkiyapi kin heon etanhan.

Wakantanka Woniya Wakan tawa kin iye tidwakinyena wakaxotexni qa ska rca wan iyeced unkicantepi kin ed ounyin kta e on ed unhipi kta iceunkiyapi waxte; heced on zitkana xikxica xni, tka ee tona Tidwakinyena kin He iyececapi, qa wankandkiya Wakantanka qa Marpiya kin etkiya kinyan kta heca ecena horpi eiqana kin etanhan hinapapi kta.

\section{WOONSPE XXIV.}

\section{WOONSPE WICOIE-WOAHOPE.}

Oekde.-Waxtewicadakapi kin he woahope yuecetupi kin hee" Boma 13;10.

W. Woahope kin dena etayhay taku Iyotan onnispe he?

A. Taku nom onmaspe; Wakantanka ekta toked miohay kte cin, qa Mikiyena-ti kin ekta toked miohal kte cin. 
W. W oahope kin tonakeca he?

A. Wikcemna.

W. Wakantanka etkiya toked niolian kte cin onspeniciye cin hena tona ee he?

A. Topa tokaheya oyakapi kin hena ee.

W. Nikiyena-ti kin etkiya toked niolian kte cin onspeniciye cin hena tona ee he?

A. Ṡakpe ehake oyakapi kin hena ee. he?

W. Toked niolian kte cin tukte wayji tokaheya

A. Wakantanka etkiya toked miolian kte cin hee.

W. Toked niolian kte cin de eced ecanol unkan unma kin ataya econ śni yaun kta oyakihi he?

A. Hiya, ecin Wakantanka Iye nopin ahopa maśi dakaś.

W. Nitanokśan uypi, qa iśta on waywicadake cin, heya waśtewicaydake śni hantanś, Wakantaıka wandakin kta oyakihi śni kin $\mathrm{He}$ waśteyadakin kta oyakihi he?

A. Hiya.

Woahope wikcemna kin hena wana onspeniciciya. Hena ta. ku akand owapi he? Tuwe napsukaza on owapi he? Tuwe onspewicakiyin kta e on qu he? Exa hena niye, qa miye, qa nakun wicaxa owasin unkowajapi. Woahope kin dena toked nioran kte cin he onspeniciya. Toked nioran kte cin tankinkinyan nonpa. Tokaheya kin he Tuwe etkiya toked nioran kte cin ee he? Qa unma kin? Oekde kin (ehin kta he?) he Wakantanka qa nitanokxan unpi kin ko owasin wastewicayadake cinhan toked nioran kte cin dena nopin terike xni yeyayin kta e heoniciyaka.

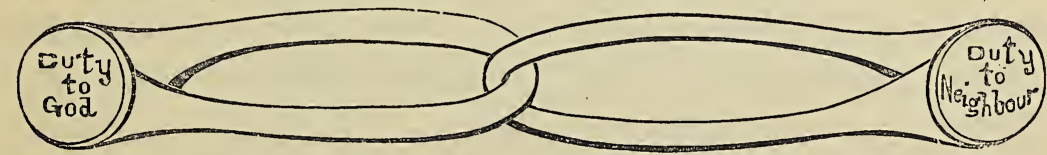

Tohinni wicaxa wanji unkan he Wakantanka cekiya kinhan on ktepi kta sdodkiya exa he itokab toked econ sa qon iyeced inihan xni econ yukan, unkan anpetu iyohi yamni Wakantanka cekiya. Heced mnaja oti timahed erpeyapi tebyapi kta $\Theta$ 
heon, tka Wakantanka mnaja kin kiunniye xni wicakiya. Wicaxa kin he Wakantanka waxtedaka, heced Iye etkiya toked oran kta iyecece cin eced oran. He caje kin omayakidaka oyakihi he? Daniel hee.

Tohinni wicincana wanji unkan sunkaku iye apa exa tahu ed adoksohan yuza unkan ikiputaka. Niye kinhan heced oyaran kta tka he? Iye kin waxteicidake cin iyeced ikiyenati kin waxtedaka, unkan heced he etkiya toked oran kta iyecece cin eced oran. Tuwe awicakehan waxteyadake cinhan, he canteyakiya se wicoie eyakiye cin iyakna toked iyokipiyayin kta e yakuwa ecee kta.

Taku yecun kta wanji ded owapj, toked nioran kte cin non. pa kin dena kiksuya yaun kta e heon-isto iyohi on napoktan heca. Maza-huhu mazaskazi qa inyan teririka on kagapi kin isanpa hena terika. Wicaxayatapi waxte David eciyapi, Psalms kage ciqon, he heya, "Nitoahope kin hena mazaskazi qa inyan teririka isanpa waxtewadaka "

Napoktan kin dena ed mazaskazi kin he tohinni wiyakpa xni ayin kte $x n i$, qa inyan terika unma "kin akand "Wakantanka etkiya toked oranpi kta" he, qa unma kin "Ikiyena-ti kin etkiya toked oranpi kia", akand heced bagopi. Nopin kicunpi kta, icir unmana kin unma kin cona un ehantanx taku wan yuxtanpi xni kin hececa.

\section{$-\mathrm{O}-$ \\ WOONSPE XXV.}

WOONSPE WICOIE-WOWAXTEDAKE.

Oekde.-Itancan Wakantanka nitawa kin He nicante ataya on, qa ninagi ataya on, qa nitowxake ataya on waxteyakidakin kta. Woahope tokaheya qa tanka hee." Matt. 22;37, 38.

W. Wakantanka ekta toked niohan kte cin, he tukte e he?

A. Wakantanka ekta toked miohan kte cin, he Iye wicawada kta, qa kowakipin kta, qa micante ataya on, qa mitawacin ataya ol, qa mitowaśake ataya on waśtewadakin kte cin; Iye ohowada kta, wopida ewakiyin kta, wowacinye mitawa kin ataya Iye ed eweknakin kta, Iye hoyewakiyin kta, Iye Caje wakan qa 'I'oie kin bduonihan kta, qa aypetu tona wani kin, owasin ed awicakehan Iye waecawecon kte cin hena ee.

W. Toked niohan kte cin nonpa tanki kinyan 
woahope wikcemna kij etanhan onnispe kin hena tona ee he?

A. Wakantanka etkiya toked miohan kte cin he, qa mikiyena-ti kin etkiya toked miolian kte cin.

W. Toked niolian kte cin tukte unma tokaheya qa iyotan tanka he?

A. Wakantanka etkiya toked miolian kte cin hee.

W. Woahope kin on Wakantanka etkiya toked niohan kte cin onspeniciyapi kin hena tona ee he?

A. Topa tokaheya oyakapi kin.

W. Woahope tokaheya kin etayhan woonspe onnispe kin he tukte e he?

A. Wakantanka wicawada kta hee.

W. Woahope inonpa kin he taku olspeniciya he?

A. Wakantanka ohowada kta hee.

- W. Woahope iyamni kin on taku onspeniciyapi kin he tukte e he?

A. Wakantanka bduonihan kta hee.

W. Woahope itopa kin he taku onspeniciya he?

A. Wakantanka wawokiya waśkan kta hee.

Napsiyordi cikciqana, niye yecun kta on ded bduha. Woahope tokaheya kin wowapi cona ehe cinhan dehand wanji cicu kta. Napsiyordi ciqana kin de ed owapi kin he taku be? "Wakantanka wicada wo", hecetu ci? Ho, nape catka yuwankand yuza wo, unkan hed napahunka ed unciyin kta. Nitawacin ixta kin on hed oniyuxtan wandakin kta oyakihi, unkan Woahope tokaheya nakaha ehe cin, qa woonspe wan hetanhan onnispe kin hena kiksuya yaun kta e oniciyin kta on he nun kta. Wakantanka Iye nicaga, qa awanniyaka, qa tenirida heced wicada yaun kta. Iye taku iwahoniye cinhan toked eye cin awicakehan eced ecanicon kta e tanyan sdodya yaun kta. Woahope inonpa hanska kin he ehin kta oyakihi he? Ho, tanyan eha; qa wana catka eciyatanhan nape tokaheya $a^{\text {k }}$ kdugata wo, unkan napsiyordi inonpa kin he hed ociyuxtan kta. He ed owapi kin he taku he? "Wakantanka ohoda 
wo." Woahope inonpa kin, he he onspeniciya. Napsiyordi kin ekta eyatonwan canna, hinhanna qa rtayetu eca wocekiye ehin kta iyececa kin he, qa Anpetu Wakan eca Wakantanka iceyakiya, qa yatan iyadowan kta e on Tipi-wakan ekta nin kta he nakun kiksuya yaun kta e oniciyin kta; icin, heconpi kin he Wakantanka ohodapi kin hee.

Wana Woahope iyamni kin eya wo. Nape cokaya on napsiyordi wan ded niciyanka. He akand iapi kagapi kin he
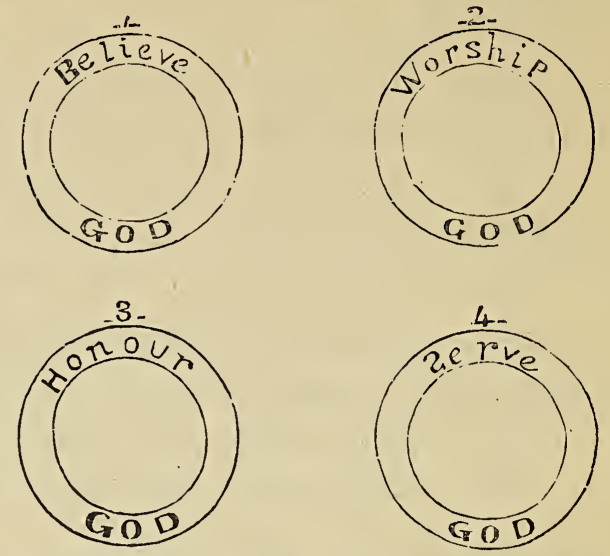

taku he? "Wakantanka yuonihan wo." Wocekiye eha canna, toked ehe cin wicoie iyohina wicayake rca e, qa Wakantanka naniron yanka he kiksuya yaun kin hena on Iye duonitian kta nyakili. Hokxina xikxica hunr econpi sa kin iyeced nix tohinni 'laisu Wakan iyukcanxiyan qaix irad cajedatin kte xni, tka ee yuonihanyanked qa ho awanikdagya se heced ece Wakantanka Caje Wakan kin He ehin kta. 'T'ohan, Wowapi Wakan kin anpetu iyohi onxpa dawa kin he on Wakantanka Toie duonihan kta, unkan toked oyakihi kin ijehan Wakantanka ohoyada kta e on Wacekiya de cin he on Tipi-wakan tawa kin he duonihan kta. Wana Woahope itopa kin he naronunya wo. Hehe! De taku akiktonx iyeuncecapi sa heca, dakax otokahe ekta "kiksuya wo" eya, Napsiyordi itopa kin he xaxte iyokihe kin akand unkoyuxtan kta. De akand heced owapi, "Wakantanka wawokiya un wo", unkan Anpetu tawa kin wakanyan yuhapi kin he; qa Anpetu-wakan canna wowaxi qaix woxkate unmana econpi xni; qa Anpetuwakan oko kin ataya lye toked iyokipi kta iyecedya xkanpi kin hena on Wakantanka wowaxi ecakiconpi kin hena ee e Woahope kin de onspeunkiya. Heced ecanon kinhan enpetu iyohina Iye wawoyakiyin kta oyakihi, unkan heced nikduha kin hecena on eciyatanhan awicakehan ohinni canteniwaxte kta oyakihi. Napsiyordi kin dena nun kta akiktonje xni wo - hena yecun kin he kiksuya un wo, qa wicoie hena akand owapi kin, qa on toked kapi kin koya awacin un wo. Tokata Anpetu-wakan kinhan nakun taku dececa nun kta e cicibduha kta. 


\section{WOONSPE XXVI.}

WOONSPE WICOIE-WOWAXTEDAKE.

Oekde.-."Qa inonpa kin he iyececa; Nikiyena un kin he niye inikdacin waxteyadakin kta ce." Matt., 22;39.

W. Nikiyena-ti kil ekta toked niolian kte cin tukte e he?

A. Mikiyena-ti kin ekta toked miohan kte cin, he miye iyeced waśtewadakin kta, qa toked ecamiconpi wacin kiı, he iyeced wicaśa owasin ecawicawecon kta: Ate qa Ina waśtewicawadakil kta, wicabduonihay kta, qa owicawakiyin kta: Woope-kaǵapi kin hena anawicawaǵoptaly qa wicabduonihay kta: Wicaśa itaıcaupi mitawa kin, waolspekiyapi kin, qa waawayyaka wakaypi kin, qa mayuhapi kin owasin ed wicihukud iyemiçyin kta: Tona misaypapi kin hena ed yuonihanyan onsiiçiya miolian kta: Tuwena oie qa oliay on kiumniway in kte śni: Miolian kin owasin ed owotanna qa wicakeya waun kta: Micante kin ed taku śica qa wokipajin bduhe kte śni: Minape kin waicucupi qa wamanolpi etaıhäl iyog wakduha kta, qa miceji kil taku iupi-śica, woitolśni, qa waaiapi kin etayhay: Mitancan kin ijakapeiçiyapi śni, bdesahay, qa aśape śni ed wakduha kta: Wicaśa tokeca taku tawapi kin war.in qa wakon kte śni; tka ee taku on wani kte cin mikdamna kta e wicolian olsspemiçiciyin kte cin, qa Walkantanka wiconi mitawa ed, wicolian kin tukte etu kaśa ed mi o kte cinhay he ed iyokipiya owotanna miolian kte cin hena ee.

W. Woahope kil etayhay toked niohal kte cin tayka wayji tokaheya onnispe kin he tukte e he?

A. Wakantanka waśtewadakin kta hee.

$W^{\top}$. Nakuy he iyececa toked niolial kte cin wayji iyokiheya hetayhay onnispe kin he tukte e he? 
A. Tuwepi kaśa wicaśa owasin. waštewicawadakir kta.

W. Woahope kin Nikiyena-ti kin etkiya toked nioliarj kte cin e onspeniciye cin hena tona ee he?

A. Sakpe ehake oyakapi kiry hena ee.

W. Woahope izaptan kin he toked nikiyena-ti ki门 waśteyadakin kta e onspeniciya he?

A. Ate, qa Ina qa tona miwankab unpi kin hena owasin wicayuonihar maši.

W. Woahope is̉akpe kin he toked tuwepi kaśa wicaśa owasin waśtewicayadakin kta e onspeniciya he?

A. Hena warjina kiumniwaya, qais̉ kiunıiyanpi kta waciク kte śni ce emakiya.

W. Woahope išakowin kin he taku onspeniciya he?

A. Micante qa mitancan kiø hena as̉ape śni mikduha kta.

W. Woahope išakdoğan kin etaghan taku onnispe kin he tukte e he?

A. Wicaśa tokeca taku tawa kinhar iwacu kta iyececa s̉ni.

W. Woahope inapcinwanka kin Nikiyena-ti kin etkiya niolian kte cin wanji onspeniciye cin he tukte e he?

A. Wowicake ece iwayin kte cin hee.

W. Woahope iwikcemna kin he toked nikiyenati kin waśte adakin kta e onspeniciya he?

A. Taku tawa kin wayjina wakon kte śni e he olsspemakiya.

W. Anpetu kin de Oəkde tawa kin he toked caje qupi he?

A. "Woope mazaskazi kin", eya cajeyatapi. Hekta Anpetu-wakan taku ociciyake ciqon hena ayektonje 
xni hantanhanx, nape cikciqana kin dena nahanrcin napsiyordi ikoyag un. Hena tona ee he? Napahunka akan napsiyordi kin he taku eya he? Qa nape tokaheya kin akanb nun kin he taku eya he? Nape cokaya akand nun kin he taku eya he? Saxte iyokihe kin akand nun kin he taku eya he? Unkan wana ninape unma kin owasin on napsiyordi bduha. Tokaheyah in he catkantahan xaxte ed unkoniyuxtan kta. He "Waanagoptan un wo," heced ed owapi. Woahope wan waunagoptan yaun kta onspeniciye cin he ehin kta oyakihi he? Izaptan kin hee.

Ho, tanyan eha. He niyate qa nibun, qa Wicaxa-wakan qa waonspekiye nitawa kin hena anawica ;akigoptan kta e onspeniciya, qa makoce wowasukiye kin Tunkanxinayapi qa tona oitancan unpi kin ko owasin, canksa-yuhapi kin eepi daex nakun, oyate kin ahopewicakiyapi kta kuwapi kin hena
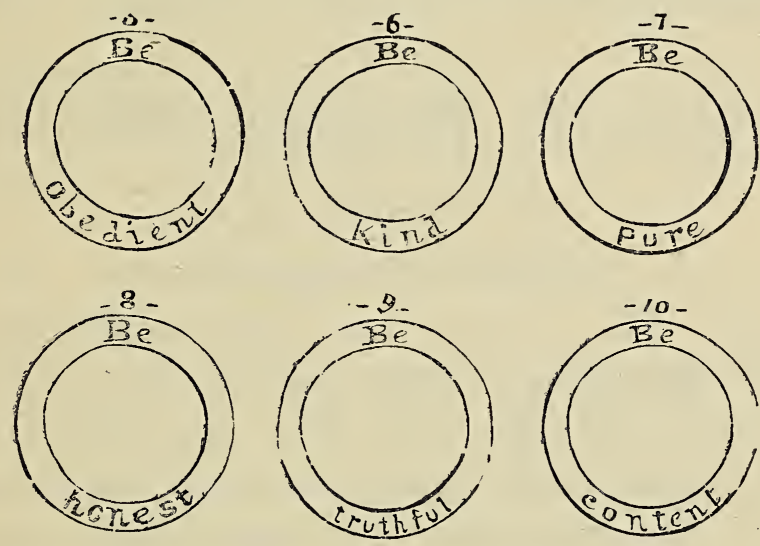

koya anagoptan onspeniciya. Wana nape onspeyapatanhan yuwankan yuza wo, unkan napsiyordi iyokihe kin he napahunka ociyuxtan kta He "Waonsida un wo" heced eya, rnkan Woahope ixakpe kin wana ebin kta, he tuwena waapa qaix kiuniye xni unkonpi kta, tka ohini wicaxa tokeca kin cantewaxtewicunyanpi kta e unkuwapi ta he onspeunkiyapi. Nape tokaheya kin akand napsiyordi wan "Wakaxotexni un wo" eya owapi kin he hed unkoyuxtan kta. Woahope ixakowin kin he eya wo. De on tancan xape xni nikduha kła he, qa woyute qa wayatkanpi kin on iyakapeniciyin kte xni e, qa nioran qa nioie kin owasin wakaxotexni qa wakitan xni yaun kta hena onspeniciya Qa nakun unkicantepi kin woawacin xikxica kin takuna on unxapapi kte xni e Wakantanka iceunkiyapi kta iyececa. Nape cokaya on napsiyordi kin he "Owotanna oran "No" eya, unkan Woahope ixakdogan kin he wana ehin kta. He taku kaxa tuwe tokera tawa kinban, tanka qaix ciqana exa, iyacu lite xni e onspeniciya. Xaxte iyo- 
kihe kin he napsiyordi wan "Wowicake ece eya wo" eya onspeniciye cin he yuhakta-tuwena qaix takuna rein owekiwakan xni wo. Woahope inapciwanka kin eya wo. Unkan napsiyordi ehake kin he xaxte tawa. He akand taku owapi he? "Iyokipi un wo." Woahope iwikcemna kin eya wo. Woahope ehake kin de cante awanikdag yaun kta onspeniciya, heced tuwe tokeca taku kaxa tawa kinhan he cantoyakpani kte xni e heon. Iyokipi yaun kinhan, taku tona Wakantanka nicu kin hena owasin on icanteniwaxte kta, unkan niye toked sdodyakiye cin isanpa Iye taku tona duha kta iyececa kin hena sdodniciciya e heced eein yaun kta.

Wanyaka po - Wakantanka qa Iyokihe-ti kin ektakta toked oranpi kita e, qa "Itancan Tawocekiye kin ed Wakantanka taku yakida he?" hena on woonspe kin on Woahope wikcemna qa Itancan Tawocekiye kin on akta kipiya onspewicakiyapi sececa. Heon etanhan Akta Wiwicawangapi ded sanpa takuna kagapi xni.

\section{ONXPA IV. GHRISTIAN WOGEKIYE.}

\section{WOONSPE XXVII.}

WOONSPE WICOIE-KIDA.

Oekde.-"Da po, kinhan nicupi kta." Matt., 7;7.

W. Hokśiyopa waśte mitawa kin, he de sdorya wo; dena niye cinka ecanon kte cin oyakihi kte ślji, qa naknn lye towaśte iyotrn kin niçu śni kighay, Wakantanka Woahope tawa kin hena okna mayani, qa tawacin kin oyapa kte cin oyakihi kte śni; heon he ohiyni wocekiye eciyatanhan bdihedya yakida kte cii, oyspeniçiciyin kta. Heon etanhay, Itancan Tawocekiye kin eha oyakihi hecinhan, nahonmayan wo.

A. Ateunyanpi, malipiya ekta nayke cin, Nicaje wakaydapi nuywe. Nitokiconze u nuywe. Mahpiya ekta nitawacin econpi kin. He iyeced maka akand econpi núnwe. Aypetu iyohi ağ uyapi kin, appetu kin de unqu miye. Qa tona ecinśniyan ecaunki onpi kin wicunkicicajujupi kin, he iyeced waunlitanipi kin unkicicajuju miye. Qa taku wawiyutan kin ekta un- 
kayapi śni ye; Tka taku śice cin etạhạ eugłdaku miye. Amen.

W. Wocekiye kin de toked ec yapi he?

A. Itaycal Tawocekiye.

W. He tokeca e [tancan Tawocekiye kin eciyapi he?

A. Itaycan unkitawapi, Jesus Christ, Iye he unqupi kin heol etanhal.

W. Wocekiye kil de ed Wakantanka toked cajedata he?

A. Ateunyalpi.

W. Wocekiye kin de on, tokaheya unkidapi kin he taku he?

A. Wicaśa owasin hin cante on Wakantanka ohodapi kte, ça wawokiye śkaupi, qa anag̀optaıpi kta hee.

W. He iyohakab unkiś unkiyepi kin on unkidapi kin he taku he?

A. Wakajtanka woyute qa hayapi unqupi kte, ça waunhtanipi unkicicajujupi kta, unkan wośice owas etanhan itokan unyuhapi kte cin hena ee.

W. Taku kin dena niśnana śni, tka nakun tuwe tokeca koya ol yakida he?

A. Ho, Wakantanka cinca kin owasin wicawecicida.

W. Taku yakida kin hena niçu kta okihi he? kta.

A. Ho, icin lye hesa, "Da po, kinhan niçupi

Tohinni wabdenica hokxina wan Leonard eciyapi, unkan nina warpanica. Christmas can-ekdepi wan waxte, qa woyute qa hayapi sanpa ota yuha kta nina cin. Heced Itancan Jesus Christ wowapi kicaga, unkan toked icakije cin he okiyake ca taku cin kin hena owasin kida. Wowapi kin tiwankan janjankdepi kin etanhan kud erpeya, unkan Itancan kin wowapi ciscina qon heced iyayeya dakax winyan jica wan, canpakmi waxte tawa kin etanhan wanyaka, unkan hokxina taku icaki- 
je cin hena qu kta $\Theta$ awicakehan Wakantanka heced ikiciyukcan kecin. Heced can-ekdepi wan wanapixkanye qa canhanpi-xaxa ojuna, hayapi tawanji coza wan, qa woknake woyute ojuna, heca wan ko opeton, unkan "Itancan kin Leonard ciqana taku hiyukiye cin hena dee" heced akapatanhan owa qa Christ tonpi anpetu itokab rtayetu kin ehand kokxina ti kin tiyopa kin ed eknake ca kikda... Tka unkiyepi kin Wakantanka wowapi unkicagapi kta iyececa xni. Wicoie unkeyapi kin hena owasin rcin naron, qa woa acin unkitawapi kin hena e kaex nakun sdodya ye do. Tohinni wicaxa wan unkan hancokaya ehand tuwe icimani ed hi, tka woyute qu kta takuna yuhe xni. Heced ikiyena ti wanji ti kin ekta i, qa tiyopa ed hehan katoto. Ohanketa ikiyena-ti kin kikta, unkan aguyapi xpan yamni oqu kta kida, takodaku wanji icimani ed hi kin heon etanhan. Tka ikiyena-ti kin ayupte, ca

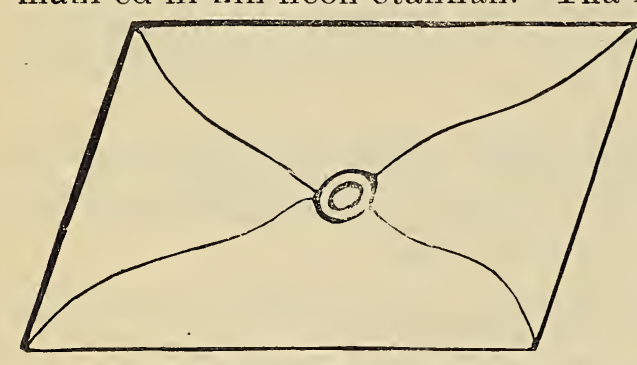
wana iwanka keya, nakuntiwahe tawa ixtinnmapi, qa tokanna hehand inajin qa qu kta okihi xni se eciya. Wicaxa qon hecekna wakida kt: ayaxtan he? Hiya. Hecetuwanjica tiyopa katoto qa wabida najin, eced ilsiyena-ti kin naron iwatuka kin on inajin qa taku cin qon owasin qu. Wakantunka taku uncinpi kin hena unkidahanpi exa tohinni naunronpi kin iwatuke xni.. Takuke ceyax unkidapi kta unkokihipi, unkan he unkiwaxtepi kta hantanhanx, awicakehan unkicupi kta.

Heced Wakantanka tatiyopa kin hed ijehan unkatotopi kta, icin "Da po, kinhan iyacupi kta" ce heceknana eye xni, tka "Ode po, kinhan iyeyapi kta; katoto po, kinhan niciyurdokayapi kta" ce, nakun heya.

Tiyata rtayetu qa hihanna owasin, qa Anpetu-wakan canna Wakantanka 'lipi-wakan tawa kin okna wayakida kte, ca waayakite, ca wayakatoto kta iyececa.

\section{WOUNSPE XXVIII.}

WOONSPE WICOIE-WOCEKIYE.

Oekde.-"Wocekiye mitawa wazintonpi kin iyeced nitokab un nunwe." Ps. 141;2.

W. Wocekiye kin de ed Wakaytanka taku yakida he?

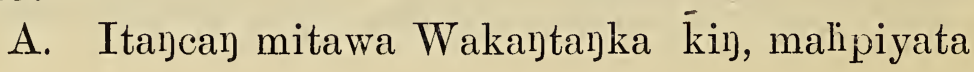


Ateuljyaypi kin, he iye wowaśte owasin wicaqu kin, miye, qa wicaśa owasin wowaśte unkukiyapi kte cin he wakicin; heced iye ceuıkiyapi qa tolian econkuıpi, qa iye anaungopta! pi kta iyecece cin, iyeced econkunpi kta. Qa nakuy uınaǵipi qa untaycaypi kin taku icakije cil hena ulkukiyapi kta e Wakantanka icewakiya; he onśiundapi, qa woahtani unhapi kiı uykicicajujupi kta; qa unnaǵ̀ipi qa untancaypi kin ed taku wokok pe kil owasin ed uykikiyapi qa ounkiyapi kta he iyozipi kta; qa woalitani qa wicolian śica owasin etayhay, qa woniya-sica tokaulyaypi kil etajhal, qa wiconte owihanke wanice cin hena etayhan iyog unyuhapi kta, hena icewakiya. Qa de eced econ kta wacinwaya, towaonsida qa towaśte kin he eciyatanhal, Jesus Christ Itancanunyaypi kin eciyatanhay. Qa heoy etayhay, Amen, hecetu nuywe, epe.

W. Tohan taku yacin qa Wakantajka yakida kinhay, he taku eciyapi he?

A. Wocekiye.

W. Taku yacin śni kin heca Wakantanka yakida kinhay, he wocekiye heca he?

A. Hiya, taku wakida kinhay he micante on wacil kta iyececa.

W. Itancan tawocekiye kin on taku yakicin kin he tukte e he?

A. Wakantanka towaśte kin he, wicaśa owasin Iye iyokipiyapi kta e heop.

W. Nakuy taku yakicin he?

A. Wakantanka wośice owasin etanhan iyog uyyuhapi kta, qa taku unyuhapi kta iyecece cin owasin unqupi kta, hena ee.

W. He tuwe e taku waśte kin dena yakida he?

A. "Itancan mitawa Wakantanka kin, malipiyata 
A teunyanpi kin, he iye wowaste owasin wicaqu kin" hee.

W. He tokeca e niye niśnana śni, tka wicaśa tokeca koya taku waśte kin dena wicaye $\cdot i c i n$ he?

A. Temiciliida kin iyeced tewicawahida kiıhan, taku waśte kin owasin yuhapi kta e wacin kta, heon etaljhay.

W. He tuwe e on etayhan Wakaytayka nanilion kta he?

A. Itaycanuyyaypi Jesus Christ, Iye on etayhay.

W. "Amen" eyapi kin he on toked kapi he?

A. Tokin, toked icewakiye cin hecetu kta nin! Heced kapi.

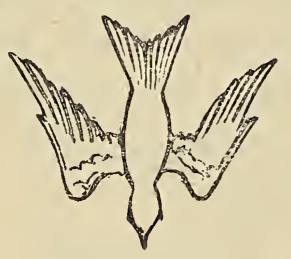

Wakxica ciqana wan ded otkejahan owapı kin, he inazazi on kagupi. He jyakarpe kin alaya ordoka ciqana ojuna. Peta kin etanhan letaga wanji icupi qa waksica kin okna!sapi, qa hehan ta'su canxin izidyapi kin Leca onxpa petaga kin

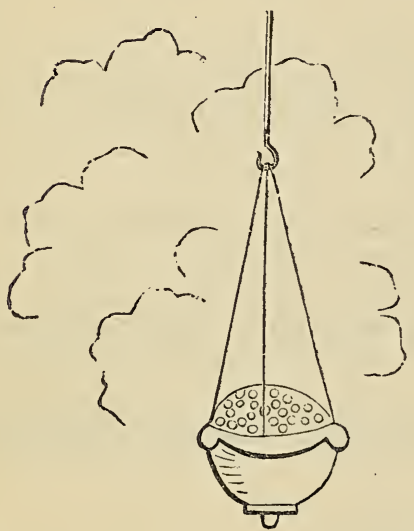
akand eknakapi. Nina waxtemua, unkan ide aye cin icunhan xota kin iyakarpe kiu ed ordoka kin okna wankand hiyu, eced tipi kin alaya woomna waxte ojund. I'ka maka ikceka onxpa wakxica kin oknakapi kinban, ide kte xni, unkan woomua waxte wanicin $k t a$. Oekde unkitawapi kin he taku-izidyapi kin on taku eya, ci? He taku e "wazintonpi kin" iyececa eya he? Ho, Wakantanka ceunkiyapi, unkan icunhan taku unkiihapi kin ol unkidapi kin hena uncantepi kin on uncinpi kinhan, wocekiye lín he wankand Wakantanka etkiya iyaya, qa Iye elata taku waxtemna heca. Tka wocekiye wan wicoie kin aecena Wakantanka unkekiyapi, unkan icunhan taku unkeyapi kin he awauncinpi xni, qaix taku unkidapi kin hena unkicupi xni exa toka undapi xni hecinhan, he maka ikceka onxpa xota waxtemna takuna Wakantanka ekta wankand iyayeye xini kin iyececa. Tohan wocekiye kin, taku-izidyapi kin iyeced, 
wicakapi cannahan, Wakantanka ohinni woayupte wicaqu ece, unkan woayupte iyotan waxte kin he Tidwaikinyena Wakan tawa kin hee, he Towaxte kin ee, unkan He u qa unkicantepi kin ed oti kta, lye iyokipiunyanpi kta e ounkiyapi, qa taku unqupi kin hena owasin ipidaunyanpi qa icantewaxteun. yanpi kta e on unkiyepi ed ounyin kta.

\section{ONXPA V. GHRISTIAN WAKIGONZAPI. WOONSPE XXIX.}

WOONSPE WICOIE-WANIKIYAPI. $119 ; 117$.

Oekde.-“Yuwankan mayuza ye, kinhan wani kta." Pe.

W. Christ Okodakiciye tawa kin ed Wakiconza: pi tona kağa he?

A. Nomnana, henaos on wanikiyapi kta; Baptisma qa Itancan Rtayetu-wotapi tawa kin hena ee.

W. He tuwe e miniunkakaśtaypi kta keye ca Itancan Rtayetu-wotapi tawa kin icu unśipi he?

A. Jesus Christ Itancal unkitawapi kī Hee.

W. Wakantanka toked econuyśipi kin eced econkoypi kta tawatedunyaypi śni ehaytanhayś, unkiye $\mathrm{He}$ waśteundakapi he?

A. Hiya.

W. Unkiye Wakantanka waśteundakapi kin he toked unkdutaninpi kta unkokihipi he?

A. Anauggoptaypi kin he on.

W. He tokeca e Wakiconzapi kin dena uyyuhapi kta iyececa he?

d. Waniunkiyapi kta e heon etayhay.

W. Wicaśa owasiu hena y uhapi kta iyececa he?

A. Ho, maka owancaya tona hena icu okihipi kin owasin icupi kta iyececa.

A. Noypa.

W. Wakiconzapi kin tonakeca he?

W. Tokaheya kin he toked eciyapi he?

A. Baptisma.

W. Unma kin iś toked eciyapi he?

A. Itancan Rtayetu-wotapi tawa kin. 
Tohinni wicaxa waxte wan Noah eciyapi. He ehand maka akand unpi qon wicaxa kin he ixnana rein Wakantanka waxtedaka qa kokipa. Unmapi kin owasin nina oran xicapi ree cin on etauhan Wakantanka hena minitan fanka on ihangwicas in kta keya. Exa Noah tiwahe ko owasin hena niwioayin kta eya. Heced Wakantania Noah ark wanji kar xi, he wata tanka iyececa, qa mini akand okarbog iyaya okihi kta. Noah Wakanianka toked econ xi qon ecerced econ, unkan ark kar un qon icuuhan, oyate okxan unpi kiu hena wahokonwicakiye ca heced xicapi kte xni icewicakiye, ca nakun iyopeic i y a pi xni ehautanx minitan wan u qa ihangwicayin tka owicakiyaka. Exa itkob i arapi, qa taku ewicakiye cin wicoie wanjina kexa wicadapi xni. Hececa exa Noah hecena ark kin kar ava, unkan hecon kin on wowacinye tawa kd utanin, icin Wakantanka

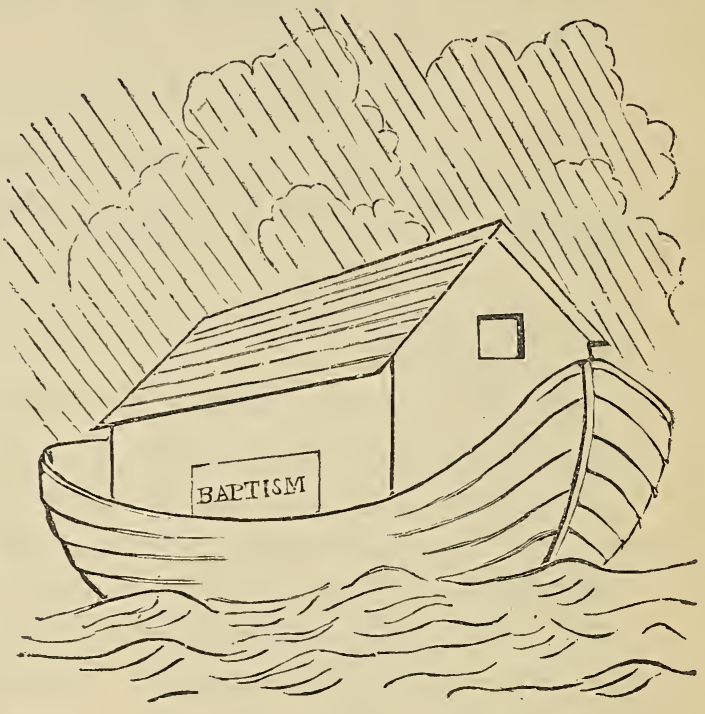
taku eciye ciqon owasin wowicakedaka. Ohakab waniyetu ota unkan wana ark kin yuxtanpi. qa Noah liwahe tawa kin ob, qa wamakaxkan ota nakun he mahed iyayapi, unkan Wakantanka tiyopa kin tuwena he yugan okihi kte xni e heced eced iyeya. Hehan magaju aya, unkan anpetu wikcemna topa hanhepi koya ihuniyan magaju, qa mini kin owancaya sam xbe aya, qa sanpa qa sanpa wakand au, eced unhanketa taku owasin mini on akarpapi, paha wankantuya koya, unkan wicaxa owasin minin tapi, Noah qa tora he kici unpi kin ixnana okatapi, unkan hena ataya waniwicakiyapi. Waniyetu wanji ataya, maka kin ake tanyan puze cin hehanyan, Wakantanka ark kin okna woyute wicaqu. Noah Wakantanka anagoptan xni unkanx ix eya unmapi qon ob ihangyapi kta tka.

Iho, maka kin de miniowanca tanka wan iyececa, qa Ark hed akand okarboka un kin he Wakantanka Okodakiciye tawa kin hee. Vinianicaxtanpi qon ehand Ark kin de ed itimaded inicupi (icin, Baptisma he tiynpa kin ee), unk:-n hed okna Wakantanka Itancan Rtayetu wotapi tawa kin on wodniyin kta. Noah ark tawa kin okna tanyan wanikiye ciqon he iye- 
ced Wakantanka tohanyan yani kin ihunniyan Ark kin de okna okope wanin niyuha kta, eyax Noah waxte waanagoptan un qon he iyeced nix eya Iye anayagoptan hta, unkan anpetu wan inicihan ed makoce owanyag waxte tukted Wakantanka ounye cin he hutata anihipi kte ca hed owihanke wanin yati kta.

\section{WOONSPE XXX.}

WOONSPE WICOIE - WOWAKTA.

Is. $7 ; 14$.

Oekde.--"Itancan kin Iye rinca wowakta wan nicupi kta,"

W. "Wakiconzapi" ehe cin he wicoie oly toked yaka he?

A. Taymahed woniya etayhay wowaste uyqupi kil, he akantalhal taninyan yutaninpi e he waka; Christ Iye linca e kaģa, he eciyctayhay he unkicupi, qa wowakta oy sdoduyyaypi kta he hececa waka.

W. Wakicolzapi he taku he?

A. Wowaśte unqupi e wowakta heca.

W. Wowakta he taku he?

A. Taku iśta ol waıbdaka owakihi, unkay taku wayyagpica śli ụ kin ol onspemakiyin kta heca.

W. Wakicolzapi way he wowaśte unqupi e wcwakta heceknana he?

A. Hiya, nakuy ol eciyatalhay wowaśte unqupi kin heca.

W. Qa nakuy taku he?

A. On wowestte wana uyqupi kin e isdodyeunkiyapi heca. he?

W. Ded wowaśte unkeyapi kin on toked unkapi

A. Uynagipi kin on wawicaqupi wašte heca.

W. Wowakta qa wowaśte kin hena unqupi kin He Tuwe he?

A. Itajcan kin Iye lica e hecol]. 
W. Wowakta kij yuha unsisip kin He Tuwe he?

A. Jesus Christ Itancal unkitawapi kiך.

Ded owapi kin he taku he? Wiknunke kin ataya wanunyakapi unkokihipi unkanx, minape ed mazanapcupe kin de iyeced kmiyan e unkabdezapi kta tka. Iho, mazanapcupe kin he wowakta heca-taku otokahe wanica qa owihanke wanica e wowakta heca, Icin mazanapcupe kin de qa wikmunke kin hena hececa, qa taku hececa kin awacinunyanpi, hera ed otokahe qaix owihanke exa takuna iyeunyanpi xni dakax... Nakun mazaxana kin de etanhan taku onspeunki-

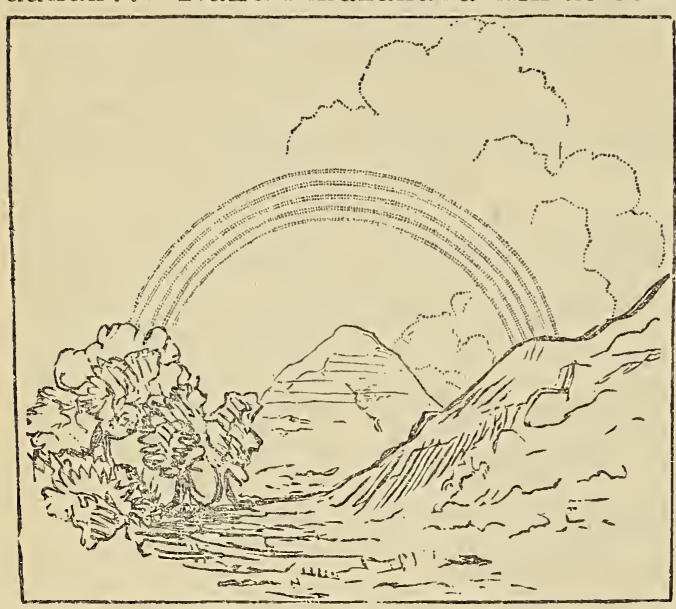
ciciyapi kta unkokihipi, Tohinni wicaxa wan wicincana nonpa iyohina mazaxana wanji wicaqu wanbdaka. Hakakte cin he wicincana nina ciscina. He mazaxana tawa kin ed etonwan qa i oknaka, qa he ha $n$ oyud waxte xni kin heon makata erpeya. 'Tka wicincana unma kin mazaxana tawa kin icu qehand ira, unkan tanyan awankdagya sican o p i y e okiknaka. Icin he kitanna waniyetu ota heced mazaxana ciqana kin he taku wan on eciyatanhan canhanpi xaxa waxte onge icu okihi hta heca e sdodya dakax.

Wakiconzapi wan ed Wakantanka wowakta unqupi kin, he ix eya taku on ecjyatanhan unnagipi kin on wowaxte Wakantanka unqupi kta cin kin hena unkicupi kta e heca. Hekta Anpetu-wakan onspeciciye ciqon, wicaxa waxte wan on wociciyake ciqon he yeksuya he? Ded iteowapi kin he he kiksuyaniyan he? Noah ark kin etanhan kdinapa qonhand, Kakantanka ohoda, unkan Wakantanka icinonpa on maka kin ihangyin kta e minitan kte xni e heced iwahoya, qa Wakantanka Noah taku wanji qu heced magaju canna he ekta etonwe kta e heon etanhan, be Wakantanka toked eciye ciqon awicakehan eced ecakicon kta e on sdodyekiya wan heca. Wowakta qa on wasdodyekiye ciqon, he wikmunke kin ee. Noah Wakantanka woiwahoye tawa kin wowicakedaka, unkan marpiya ekta wikmunlse kin ekta etonwe kte, ca Wakantanka iye taku okokipe kin owasin etanhan awanyakin kta e heced ecin un kta waxtedaka. Nix eya Wakantanka cinca henica. On wasdodyewicayapi wikmunke kin, he Wakantanka woiwahoye tawa awacinniyin kta iyececa. Wakiconzapi nonpa kin ed nakun taku waxtexte iwahoniyan, unkan Bap- 
tisma qa Itancan Rtayetu-wotapi tawa kin hena nupin ed taku taninyan wandake cin he, Wakantanka taku eye cin eced econ kta e, on sdodyewicayapi wan heca, wikmunke kin iyeced,

\section{WOONSPE XXXI.}

WOONSPE WICOIE-WANYAKA.

Oekde.- "Ixta mayukawa ye, kinhan nitoope eciyatanhan taku wowinihan kin hena ed ewatonwe kta." Ps. 119;18.

W. Wakiconzapi ed tonakiya taku yanka he?

A. Nonpa; akantanhay taninyan yanke cin hee, qa mahedtayhay woniya eciyatayhay wowaśte kin.

W. "Akantanhan" qa "taninyan yanka" eyapi kin, hena nopin owayjina kapi he?

A. Ho.

W. Wakiconzapi way ed akaytanhan qa taninyan yanke cin he taku he?

A. Iśta on waybdaka awakihi kin hee.

W. "Mahedtayhay" qa "woniya eciyatanhay" eyapi kin hena jopin owayjina kapi he?

\section{A. Ho.}

W. Wakiconzapi wan ed mahedtanhan qa woyiya eciyatanhay kin he taku he?

A. Taku iśta on waybdaka owakihi śni kin hee.

W. Wakiconzapi wan ed taku nonpa yuke cin hena tona ee he?

A. Wowaktakin hee, qa wowaśte kin.

W. Wakiconzapi way ed wowakta kin he akaytayhay qais mahedtanhay, tukte unma he?

A. He akaytanhay, icin waybdakin tka owakihi dakaś.

W. Wakiconzapi way ed wowaśte kin he akaytanhay qaiś mahedtanhay, tukte unma he?

A. He mahedtanhay, icin waybdakin kta owakihi śni dakaś. 
Ded yarugapi wan owapi. He onxpa nonpa kin tona ee he? Ha kin qaix co kin, tukte unma iyotan terika he? Ha wanica ehantanx co kin icagin kta he? Yarugapi owasin co skuya kin anakikxin kta e heon ha yukan, unkan ha kin he unkiyepi ekta wowakta heca, icin he wanunyakapi canna imahed taku waxte wan yukan e tanyan isdodunyanpi dakax.

'Tka nix niye kin he isan. pa nina taku wowinihan henica. Wakantanka onxpa nonpa nicaga-tancan wan qa nagi wan. Niixta wiya. kpakpa kin e, qa nisiha qa ninape xkanxkan un kin, hena ed ewatonwe cinhan, hena nitancan kin onxpa ee exa nitanmahed hena isan-

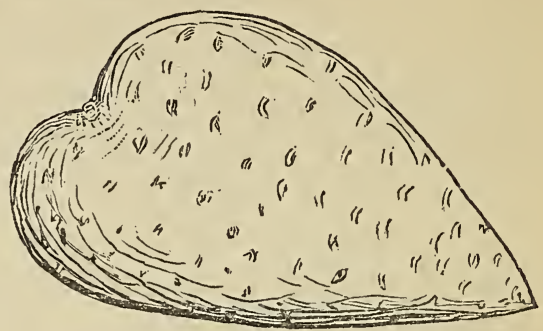
pa terika, qa niixta kin etanhan etonwan, qa ninape qa nisiha yuxkanxkan un, nagi ciscina wan yukan e isdodwaya. Iho, heced nitancan, yarugapi ha kin iyeced, taku wan wanbdaka owakihi xni qeyax hed mahed un sdodwaya e wowakta heca.

Wakantanka wakicouzapi tawa kin ed onxpe nonpa kin hena unma wabdakin kt. e wowakta nicu, unkan onxpa wandakin kta oyakihi xni kiu he nakun awicakehan hed un kin $\theta$ tanyan sdodya un nixi.

\section{WOONSPE XXXII.}

WOONSPE WICOIE-YUJAJA.

Oekde.--Niye mayadujaja kta, keced wa kin isanpa maska kta." Ps, 51;7.

W. Baptisma ed akantanhan taninyay wayyakapi kin he taku he?

A. Mini; he okna tuwe "Ateyapi kin, qa Cinhintku kin, qa Woniya Wakay kin Caje on" baptisma qupi kin hee.

W. He taku e ol mini wowidaguyyaypi ecee he?

A. Uyyuskapi kta e he on.

W. Taycal uyyuskapi kta hecena śni, tka nakuy naği uyyuskapi kta iı ececa he?

A. Ho.

W. Baptisma ed mini kil he wowakta qais wowaśte, unma tukte ee he? 
A. He wowakta ee.

W. He tuwe e mini on Baptism unqupi kta iyececa keya he?

A. Jesus Christ Itancal unkitawapi kin Hee.

W. Nakui taku eya he?

A. Baptisma unqupi kinhan ed "Ateyapi kin, qa Cinhintku kil, qa Woniya Wakal kin Caje on" miniunkakaśtaupi kta keya.

W. He tuwe caje kin ee he?

A. Trinity yawaśtepi kin, he caje kin ee.

W. I'rinity ehe cin he on toked yaka he?

A. Tuwe Yamnipi eśa Wakantanka waujjipina.

W. Baptism unqupi kin he taku abdesunkiyapi he?

A. Toked unkolianyanpi kta iyececa kin ee.

W. Toked unkohanyanpi kta iyecece cin he tukte e he?

A. Christ olianye ciqon iyeced unkolianyanpi kta.

Ninape kin nina xapa kinhan, nikduska kta e on mini on nape nikdujaja kta, tka nicante qaix ninagi xapa kinhan mini on dujaja kta oyakihi xni H+ceca exa oekde-wocekiye wan hekta onspeniciciye ciqon he nun kta oyakihi-"O Wakantanka, cante ska micaga ye" eyapi kin hee, qaix wanji decana anpehan ehe cigon he, "Niye mayadujaja kta. heced wa kin isanpa maska kta" ee. Ho, Wakantanka nagi unyuskapi kta e wowaxake yuha, unkan heced ecaunkiconpi kta e he Baptisma Wakan unqupi kin eciyatanhan on iwahounyanpi. Tohinni wicincanal wan unkan he wayaka se tawaiciye xni un kła e on wiyopeyapi, qa ti waxtedake ciqon qa atkuku qa hunku hena koya etanhan tehan ayapi. He yuhe cin iye tamakoce kin ed wicaxa itancan tanka heca, tka wicowayazan nina okokipe wan on teriya un, unkan wicincana wayaka se akdipi qon he wicaxa itancan qon tawicu wokiyaka unkan iye tamakoce kin ed Wakantanka taokiye wicaxa wan wowayazan kin he yuaxni kta okihi yukan keciya. Heced wicaxa itancan qon wowaxi wicayuhe cin iwicacu, qa taku wawicaqu teririka koya, unkan Wakantanka taokiye wicaxa ti kin ekta icimani hi. Tka wanyag hi qon he itkokipin kta e on tankad hiyu xni qa wawicaqu waxtexte kicicahi qon ed etonwe xni, tka 
hed ikiyena wakpa wan ed xakowin akikde ikdujaja kta e he. ced aecena hoyekiya. He taku oecon terike xni, tka wicaxa itancan tanka qon, nina waranicida dakax, Wakantanka tao-

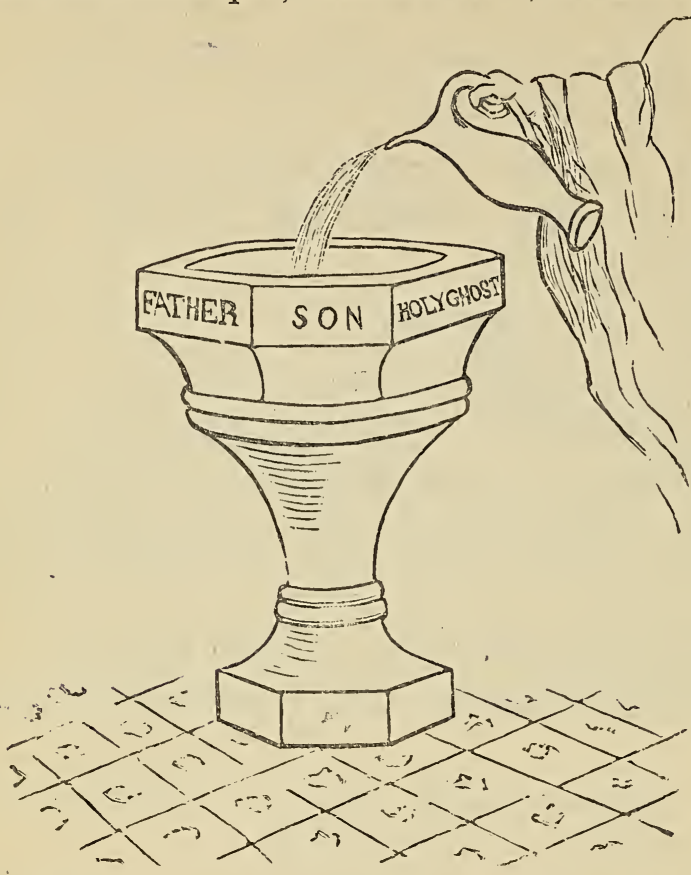
kiye wicaxa sanpa yuonihan xni kin heon xikda, unkan nina canzeka kikda. Exa wowaxi wicayuhe cin ikdu. jaja kta keciyapi qon he terike xni dakax iyutin kta icekiyapi, un k an xakowin akikde ikdujaja gonhand ataya akisni. $\mathrm{He}$ hand owasin toked icantewaxtepi ced! Unkan iye kinhan he ehand, pida rea un kin ikdutenin kta e on, takn qeyax econ qaix kajujukta e tawated. ya un. Wakantanka akisnikiya, unkan heon tohanyan ni kin hehanyan Wakantanka wawokiya xkan kta e iciconza.

Wicaxa hunr Baptisma wicaqupi kta exa he taku ciscina qa heced on iwaxtepi kta iyokibi xni ecankinpi, unkan warasicidapi on econpi kta tawatedyapi xni. Tka Itancan unkitawapi kin Iye rca e Baptisma wicaqupi kin opa unxipi, dakax Iye econ unxipi kin eced econkonpi xni ehantanhanx wowaxte iwahounyanpi qon he unkicupi kte xni e tanyan sdodunyanpi iyececa, qa unkiye taku unkiwaxtepi kta e he sdodunkiyapi kin isanpa Iye he sdodunkiciyapi.

\section{WOONSPE XXXIII.}

WOONSPE WICOIE-WICATONPI.

Oekde.-"Wicaxa kin mini qa Woniya kin on tonpi xni kinhan Wakantanka tokiconze kin $\theta d$ tid iyaya okihi kte xni." John 3;5.

W. Imahed woniya etanhan wowaśte kin he ta$\mathrm{ku}$ he? 
A. Woalitani ed tapi, qa wicoowotanna ed teca wicatoıpi kiı: ecin uıkicağapi on woalitani ed untonpi, qa wośikda cinca heujcapi, de on wowaśte cinca heca unkag̉api.

W. Baptisma unqupi kin ed unnag̉ipi kin ed toked ecaunkiconpi he?

A. Teca untonpi.

W. Baptisma unqupi kin ed teca untonpi kin he wowakta qaiś wowaṡte, uıma tukte ee he?

A. Wowaśte ki hee.

W. Unkicağapi kiı eciyataıhay on unnaggipi kin hena toketupi he?

A. Walitanipi sa, ecin woalitani ed tonpi dakaś.

W. Unkicaggapi kin eciyataıhan on tuwe cinca heuncapi he?

A. Adam cinca heuncapi.

W. Baptisma unqupi kin eciyatanhan on tuwe cinca unkaǵapi he?

A. Wakantanka cinca unkag̀api.

W. Toked Wakantanka Iye cinca unkag̉api he?

A. Wiyacinyan cincaunyanpi kta unkicupi.

W. Icag่e cin eciyatạhal on iśnana Wakantaıka cinca lice cin He tuwe he?

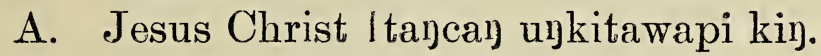

W. Baptisma unqupi kin eciyatanhan on Wakantajka wawicaqupi tanka ujqupi kin hena tona ee he?

A. Woahtani unkicicajujupi kin qa Woniya Wakal tawa kin, heya ee.

Tohinni wicaxayatapi waonxida xni wan, unkan tamakoce ed oyate tokeca wan tipi qón hena cinca hokxipina kin owasin wakpa kin ed minin tewicayapi kta keya, unkan napapi kta okihipi xni kin heon anagoptanpi kta hecena okihipica. Qa wicahunku wanji hokxicana nina owanyag waxte yuha, unkan ohinni nina cantexica un exa, cinca hokxina minin 
teyapi kta e tawatedyin kta ecaca okihi xni. Heced wi yamni hehanyan timahed anakirma, unkan hehand wata se psa-wouha ciscina wan psa on kicaga unkan hokxicana owanyag

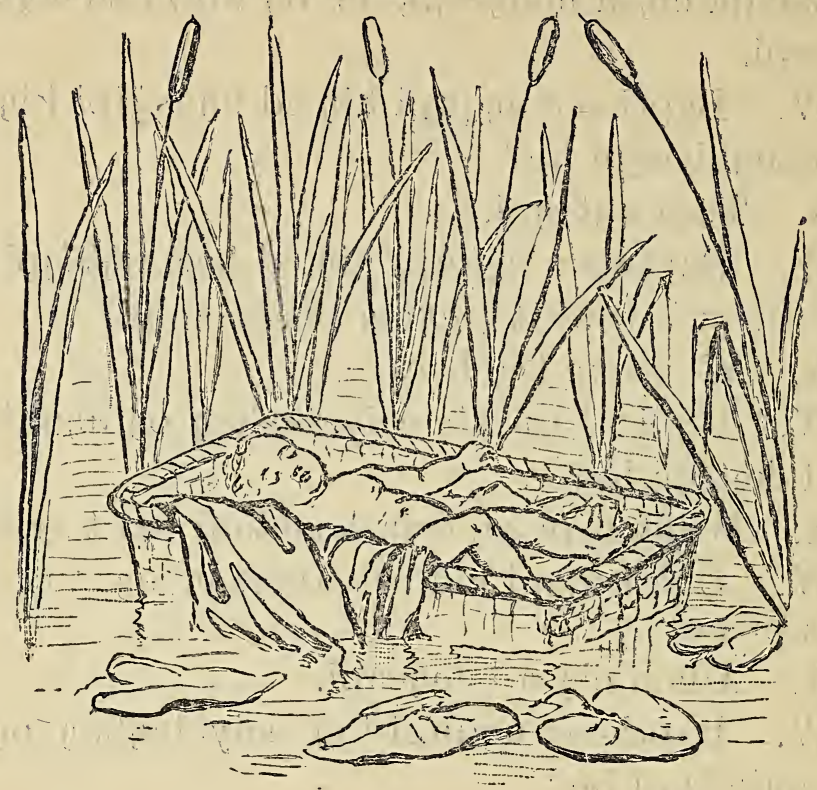

waxte kin he ed oknaka, unkan psa hanskska wakpa kin ed hutata icakda icage cin hena ekna anarma. Unkan wicaxayatapi cuwintku kin tohan ikdujaja kta e on wakpa ed hi qehand, psa-wojuha eiscina kin wanyaka unkan hokxicana ceya un kin he nina icantekicixica kin on wiyacinyan cincayin kta e icu kta keya. Toband icarkiciyapi kta eon winyan wanji ode qehand, hokxicana hunku kin he e k ta eknayan kicicahipi, unkan wicaxayatapi cuwintku kin hokxicana tanyan awanyag xi, qa wixi ota qu kta keciya. Heehand hunku kin he toker nina wiyuxkin, ci? Tanye rein awankdaka, unkan hokxicana kin tanka icaga qehand, wicaxayatapi cuwintku kin ekta ahi, unkan he wiyacinyan cincaye ca kici wicaxayatapi ti kin okna ti, qa teyapi kta ikope xni ed ounyan, ecin wana wicaxayatapi tiwahe tawa kin he ed opa dakax. Moses ciqana heced wayutokecapi wan tanka akipa, ci? Eyax nix eya wayutokecapi wan he iyeced tanka ayakipa. Niye kinhan ounye oiyokixica qa woartani ojuna, heea ed nitonpi, Adam cinca heniea, qa he Satan towaxake ihukuya un, qa niye kin owihanke wanin wiconte ed idanin ktaiyeniceca tka. Tka hunkakewicayaye cin qa Baptisma nicupi ed waeniciyapi kin, iyecinka niniyanpi kta okihipi xni kin heon okna-miniawicakaxtanpi kin ekta anihipi, Wakantanka Iye toked iyokipi kin ecanicon kta e heon. Unkan Wakantanka onxinida, unkan Baptisma nicupi kin eciya- 
tanhan on wiyacinyan cincaniyin kta e inicu. He teca wicatónpi wan heca. Wana dehand marpiyata Wicaxayatapi un kin He cinca kin henica, qa tamakoce tawayayin kta iyeniceca, unkan Iye sutaya ed ikoyag yaun ehantanx, tohand Iye ti waxte kin ed kici yati kta e anpetu inicihan. Marpiyata Ateyaye cin He toked un kin iyeced inicagin kta e yakuwa kta, qa hecanon kta e on wowaxte iwahoniye ciqon he yakida kta iyececa.

\section{WOUNSPE XXXIV.}

WOONSPE WICOIE-ECON.

Oelede.- "Xicaya econpi ayuxtan po: taku waxte econpi onspeiciciya po." Is. $1 ; 16,17$.

W. Baptisma wicaqupi kte cin hena itokab taku yuhapi kta he?

A. Woiyopeiçiye, he eciyatayhay woalitani ayus̉tappi; qa Wowacinye, he eciyatanhan Wakicolyapi kin de ed Wakaytanl:a toked ecol kta keye cil hena awicakehay wicadapi kta.

W. Woayupte kil de taku onspeulkiyapi he?

A. Baptisma uljqupi kin ed olşpa uıkiye econkolpi kta e yukay.

IV. Unkiye econkonpi kte cil he taku he?

A. Iyopeunkiçiyapi qa wacinunyanpi kta.

W. Woiyopeiçiye kin he taku he?

A. Walitanipi kin olf icanteśicapi qa etayhan ikdamnapi.

W. Waunhtanipi kin on aw:cakehay icanteuns่icapi kil he toked unkdutaninpi kta he?

A. Hena uıkokdakapi qa unkizapi kin on.

W. Wowacilyye kin he taku he?

A. Wakajtaıka wicadapi kin hee.

W. Wakantanka wicaundapi kin he toked ul)kdutajiupi kta uıkokihipi he? 
A. Wakantarka toked ecor unšipi kin eced econkorjpi kin he ors.

W. Wakiconzapi kin de ed Wakantanka iwahounyanpi kin hena tona ee he?

A. Christ urjketanharpi kta, qa Wakantarjka cinca, qa Malipiya tawayapi kin heuncapi kta, hena ee.

Hokxina wan ded owapi kin he hekta unma edkiya eciyopteya mani tka. Wicanape tanin xui wan miyokdasin wan
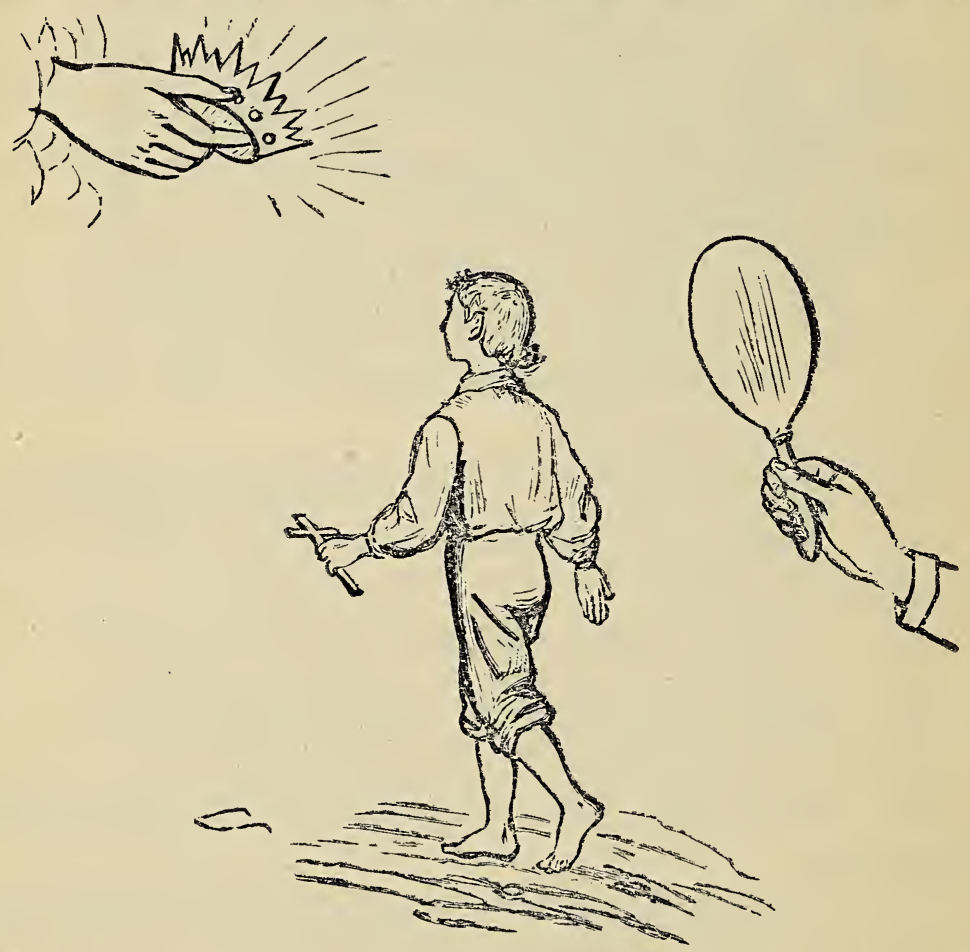

iye itokab ohinni pazo un, tka he ed taku waxtexte tanin kin hena ikiyena ya eca atanin xui iyaya ece. Exa tohinni anpetu wanji oknikde-wakan wan iye itokab ahinajin, unkan he nina waonxidaya etonwan kin on kokipin kta jyє ceca xni, qa heciya, "Taku kitanye rein yakuwa tka qon hena wanagi heca, tohinni sutaya oduspin kta oyakihi kte xni. Ikdamna wo, qa nite cin hehanyan wicayake cinhan, watexdake xnix ayin kte xni wan niciyuhapi kin he wanyaka wo." Hehan oknikde-wakan kin canicipawega wan hokxina kin nape okicikna- 
ka, unkan erpeya kikda. Hokxina kin ikdamna, unkan behand watexdake wan iwahoyapi qon he iye awankab wiyakpa yanka wanyaka. Qa he iyohakab miyokdasin kin ed wanagi tanin kin hena ekta icicawin kde kta e ijehan iyutanyanpi, exa hena kuwa qon on awicakehan iyopeiciya, unkan ed etanwan ayuxtan qa etanhan ikdamna kin on he kdutanin. Wowacinye on watexdake wan kipazopi kin be ekta wankan etonwan, unkan canicipawega tawa kduha toktakiya yin kta e okiyin kta he wowacinye on Wakantanka kida. "Christ taakicita qa taokiye wacinyepica" yaun kin, nix eya he iyeced oyaran kta iyeceea.

Tohinni Waayata-wicaxa wanji otonwe tanka wanji ekta ye xipi, Wakantanka ihangwicayin kta owicakiyakin kta e he on, wartanipi sa kin on etauhan. 'laku owicakiyake ciqon wicadapi. He Wowacinye heca. Wowaxi econpi kin he, qaxkatapi kin. qa wotapi qa wayatkanpi kin, hena owasin ayuxtanpi, unkan wartanipi qon ayuxtanipi kta iciconzapi, qa Wakantanka hena wicalsicicajujupi kta e icekiyapi rca cankpexka makakde erpeiciyapi qa ceyapi. He Woiyopeiciye heca. Unkan Wakantanka nawicaron qa wicakicicajuju, qa ihangwicaye xni. Otonwe tanka qon caje kin Nineveh ee, qa wayatawicaxa wan he ekta yexipi qon he Jona hee.

Rtayetu iyohi anpehan taku xikxica ecanon qon hena awacanni, qa hena nicicajuju kta e Wakantanka iceyakiyin kta iyereca. Anpetu iyohi W $\mathrm{W}$ akantanka iyokipiyayin kta e yakuwa kte ca wokokipe ayakipe cin hena etanhan enikdaku kta e heced ecanyakin kta iyececa. Hecanon kin on Baptisma nicupi qon ehand, waeniciyapi qon hena iyopeniciyin kte ca wacinyan yaun kta nicaje on eyapi qon, he eced ecanon kta.

\section{$\mathrm{O}-$ \\ WOONSPE XXXV.}

WOONSPE WICOIE-OKDAKA.

Oekde.--Tuwe kaxa wicaxa wicitokab miye omayake cinhan, he A te marpiya ed un kin itokab obdakin kta." Matt. 10;32.

W. Heced tokeca on, Hokśiyopa kin, cikcistinpina kin on etayhay econpi kta okihipi śni tka, baptisma wicaqupi he?

A. Waewicakiciyapi kil eciyatanhan nopin eced econpi kta keya okdakapi kin heon; qa he woiciconze kin, tohan wacinksapapi kinhay, iyepi hca ecol)pi kta iyececa.

$W^{T}$. Taku nopin eced ecoypi kta keya okdakapi qoy hena tona ee he? 
A. Iyopeiçiyapi kte ça waciuyaupi kta.

W. Hokśiyopa kin is iyopeiçiyapi kte ça wacinyaypi kta $\Theta$ he iyecinka eya okdakapi kta okihipi he?

A. Hiya, icil decana wicatorpi dakaś.

W. Tona iyepi kin eekiya woiçiconze taninyan eyapi kin hena taku ewiculkiyapi he?

A. Waewicakiciyapi.

W. Tohan oyakalinigge cinhan woiçiconze kin dena niye cinka eya oyakdakin kta iyececa he?

A. Ho, Christ wacilywaye cil) owakdakin kta iyececa.

W. He tohaytu kta he?

A. Wicayusutapi owapa kin he ehand.

W. He itokab olspeniçiciyin kta iyecere' ${ }^{\prime}$ in he taku he?

A. Okodakiciye Waka! kin Wiwicawanğapi kin he.

W. Wicayusutapi oyapa kin he ehand wawicaqupi wan tanka Wakantanka niçu kte cin he tukte $\mathrm{e}$ he?

A. Woniya Wakan tawa kin Hee.

W. Woniya Wakan kiı He taku okihiniyin kte cin he tukte e he?

A. Christ taakicita qa taokiye wacinyepica wauij kta ee.

W. Toked ecanol kta e oniciyin kte cin he tukte e he?

A. Christ wapaha tawa kil ihukuya wicaśa iyeced okicize ecamol kta; woalitani; maka kiı de, qa wakanssica hena kipjinyan.

Hekta waniyetu ota, Sakdaxin makoce etu, wicaxa car totinza heca yukanpi tka qon, unkan hena wicaxayatapi qa makoce tawapi kin teridapi rce, ca kicizapi ed ohinni wiyeya unpi tka. Wicaxa qon hena "Knights" ewica- 
kiyapi, unkan caje kin he wicaqupi kinhan ed iyohi minahanska wanji qa wacinhe se wanapin heca koya wicaqupi, he tuwe tawawicaya hecinhan he yuotanin, qa heced wicayuoni-

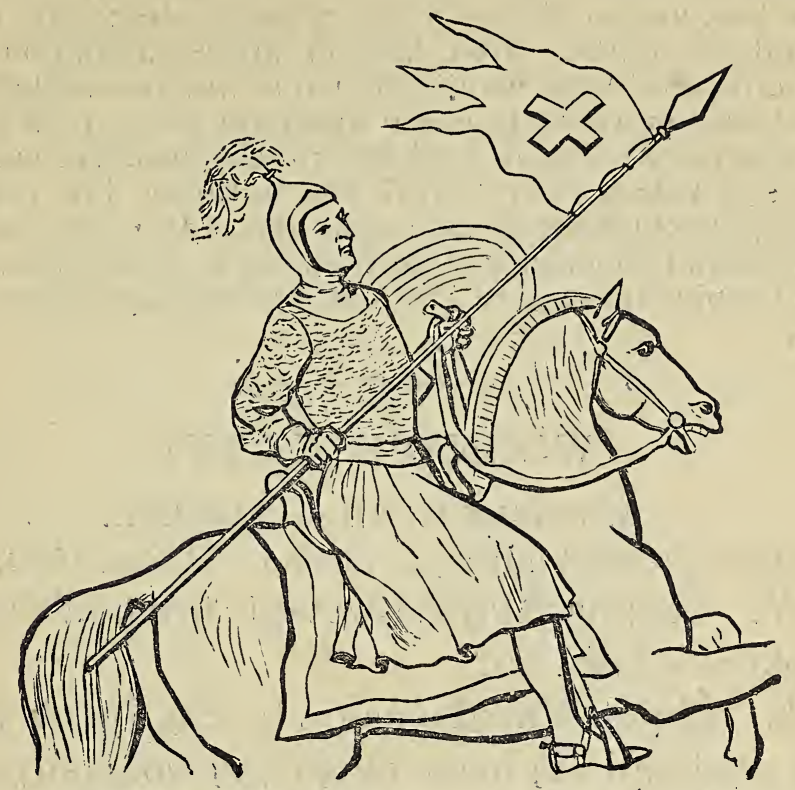

hanpi kin iwinktapi, unkan wicaxayatapi taxapi kin he anakigoptanyan unpi kta e wakanyan iciconzapi. Okde mazasuta on kagapi heca unpi. Mazawapaha koya kicunpi. Qa miwakan waxtexte tawapi kin iyakna, wahukeza qa wahacanka nakun ayapi. Zuya yapi eca toked owanyake ciqon he ded wanji iteowapi kin hetanhan ayabdezapi kta. Wicoran waditaka qa waxte otas eeonpi kin on owancaya wicayaotaninpi. Winyan qa wakanheja on xika qa iyotan iyekiyapi qon ota woterike etanhan ewicayakupi. Wicaxa mazadowankiyapi yuha tipi iyaza yatan wicadowanpi. Knights qon hena tipi-suta, castle eciyapi, heca yuhapi unkan tawicupi qa cincapi kin hena okna tipi. Cinhintku ciqapina kin ix eya tohan "wokokipe qa woiyopeye wanin" knights wicakagapi kta inarnipi, unkan on waniyetu iyenakecapi qehand knights kin ob yapi iyowinwicayapi, ihawicaktapi qa owicakiyapi kta e on, iye nakun knights wicakagapi kta ihunnipi kin hehanyan. Tokin, mix eya anpetu qon hena ed ni wann, qa knight waxte kin de iyeced, wicaxayatapi qa makoce mitawa kin nawecijin kta e on waditagya xungakanyanka kicizapi ekta bda owakihi tka nin! ecanni he? Exa Christ Wicaxayatapi nitawa He taakicita nicagapi kin wana niciyuxtanpi. Waeniciyapi kin Wapaha tawa kin ihukuya wirnma iyeced okicize ecanon kta keyapi, he akand Canicipawega tawa kin owapi, Iye ta- 
waniyan yutaninpi kta e on itehu ed wapetognitonpi qon hee. Waniyetu iyenanikeca kinhan, Wicayusutapi ecaniconpi on knight wicoran kin yakuwa kta e nicopi kta. Hehand Wicaxayatapi nitawa wokicize tawa ecanon kta iyoncipi kin he oyakdakin kta, unkan wipe Christ wiyeya niciknake cin hena heehand yecun kta. Nipa kin on maza-wapaha on okokipe wanin ounyanpi heca wanji, qa nisiha kin on wodakota canhanpa heca, qa wowaxte maku akarpapi heca, qa Wakan-xica wahinkpe tawa ontonniyanpi kte xni e wowacinye wahacanká wan, qa miwakan waxte wanji Wa antanka Jye rca ninape oniciknakin kta, hena owasin niciyuha. Iho, heon anpetu oiyokipi tohand wicoran wankantuya kin de nicupi kta, Wicayusutapi ecaniconpi anpetu kin hee, e tokatakiya ekta etonwan un wo.

\section{$--0$ \\ WOON:PE XXXVI.}

WOONSPE WICOIE-KIKSUYA.

Oekde.-- "Deced miksuya econ po" I Cor. $11 ; 24$.

W. Itaycan Rtayetu-wotapi tawa, Wakicoyzapi kiy tokeca e kagga he?

A. Christ Waikduśna qa te cin, qa etayhay wowaśte uıkicupi kị hena ohinniyal wokiksuye kta on.

W. He tokeca e Wakiconzapi kin de Itancan Rtaretu-wotapi tawa eciyapi he?

A. Jesus Itancal kin waonspewicakiye cin ob litayetu-wotapi ed yalka icuphan he econwicaśi kin heol etayhay.

W. He tohandtu he?

A. Te cin itokab hanhepi qon he ehand.

W. He tokeca e Itancan unkitawapi kin Wakicolzapi kin. de icu unśipi he?

A. Te ciqon wokiksuye heca kta e heoy.

W. Nakuı taku unkiksuyapi kta he?

A. Te cil eciyataıhan on wowas่te unkicupi sa kin hena.

W. Itancan unkitawapi kin He toked ța he?

A. Cayicipaweg̀a way ed okataypi. 
W. Hetayhạ eikdaku okihi śni dakaś heced ța he?

A. Hiya, Wośnapi heca kta e içiçu.

W. He tokeca e Wośnapi kta e içiçu he?

A. Waunlitanipi kin etanhan niunkiyapi kta $\Theta$ he oy.

W. Anpetu way ed țe ciqon he taku eciyapi he?

A. Friday Waśte.

W. He tokeca e waśte eciyapi he?

A. Christ woahtani unkicicajujupi kte ça niunyanpi kta e ol te cin heon etanhan.

Kodaniyanpi wanji mazaska wanji on wowapi teca wan openiciton kta nicu kin an, nina onxinida ecanyakin kta-wowapi kin he ed dawa kin iyena he yeksuyin kta seca. Canpakmi tkeye rcin okxupi wan anidi kta iyecetu hecinhan, un-

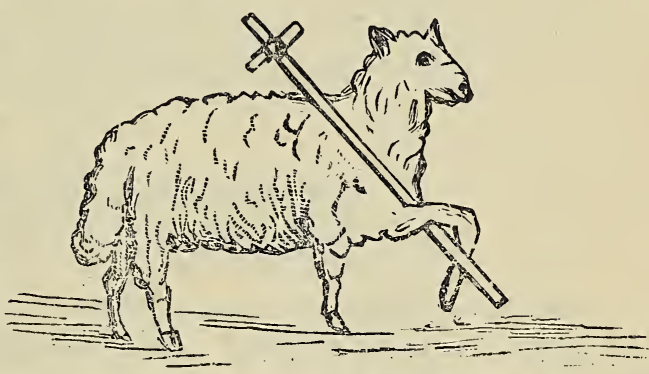

kan hokxina wan niniyin kta xkan kin icunhan isto kawegapi qa naxujapi on yuksapi kta iyececa kinhan, hokxina kin he waxteyadakin kta, ci? He taku ecanicon kin tohinni ayektonjin kta he?

Exa Tuwe Wanji he isanpa nina tankaya taku ecanicon. Itancan unkitawapi Jesus Christ He niye on ta. Niktepi kta iyecetu wanyaka, nitancan hecena xni, tka ninagi kin nakun. Heon nix maka akand okokipe wanin qa cantewaxteya yaun kta e, on Iye maka kin de ed warpanica kte, ca hehan woyazan terika ota yuba, ohakabcanicipawega wan ed okatanpi kta hena ikdonicexniyan tawatedya. Wicaxa tokeca tuwena rcin heced tankaya taku ecanicon kta okihi xni, qaix econ kta okihi exa tawatedyin kta iyececa xni. He kiksuya yaun kte, ca on Iye nina rcin teyarida kta, ci? Wanji heced kodaniye rce cin He toked iyokipiyayin kta e taku owasin on yakuwa kta, ci? He tohinni wandake xni qeyax, Iye dehand wanniyag un, unkan tokata wandakin kta anpetu inicihan.

Hekta waniyetu ota, Wakantanka cincapi kin oyate Wakantanka sdodyapi xni, qa nakun asdodyapi kta, qaix kokipapi kta awacinpi xni wan ob tipi. Hanhepi wanji Wakantanka oknikde-wakan tawa kin yexi, ikceoyate kin he tipi kin iyohi- 
na ed cincapi kin wanji kte kta qa he on iyopewicayin kta e heon. Tka Iye taoyate kin iwaktawicaya, unkan tipi kin iyohina ed tarcaxunkana cincana wanji ktepi kta, qa yutapi kta, unkan he we kin onge tipi iyohina tiyopa kin akand iunpi kta ewicakiya, heced oknikde-wakan kin he wanyake cinhan iye cincapi kin wanjina kte kte xni e heon. Oyate kin Wakantanka toked econwicaxi kin iyeced econpi, unkan oknikde-wakan hi qehan tipi kin tona tiyopa ed we iunpi kin hena wanjina timahed ye xni, tka oyate xica Wakantanka yuonihanpi kta wicadapi xni qon tipi iyohina kin ed wicate yukan. Heiyohakab waniyetu iyohina tarcaxunkana cincana ktepi, tohand Wakantanka taoyate kin wiconte etanhan niwicaye ciqon he wokiksuye kta e heon etanhan. Tarcaxunkana cincapina wicaktepi qon hena Wanikiya unkitawapi kin, Jesus Christ, $\mathrm{He}$ wiyacinpi hecapi kta e on wicaktepi. He Wakantanka Tarcaxunkana Cincana tawa kin Ee, qa $\mathrm{He}$ canicipawega kin akand wayuxnapi, niye, qa nakun wicaxa tona Iye we waxte kin ed wicayujajapi kin hena koya, owasin wiconte owihanke wanin etanhan niwicayin kta e heon etanhan.

\section{WOONSPE XXXVII.}

\section{WOONSPE WICOIE-WOYUTE.}

Oekde.-_"Tuwe ed mau kinhan tohinni docin un kte xni; qa tuwe wicamada kinhan tohinni ipuza un kte xni." John $6 ; 35$.

W. Itancan Rtayetu-wotapi tawa ed akantanhan qa wayyakapi kin he taku he?

A. Ag̀uyapi qa Miniśa, hena Itancan kin icupi kta keye cin ee.

W. Itancan Rtayetu-wotapi tawa kin ed wayunyakapi unkokihipi kin he taku he?

A. Ağuyapi qa Miniśa.

W. Hena taku eciyapi he?

A. A kantanhan qa wayyakapi ki .

W. Aǵuyapi taku on waśte he?

A. He woyute waśte, tanzani qa waśaka icahunyaypi kta e heon.

W. Miniśa taku on wasste he?

A. Tona was̉akapi śni qaiś wayazaypi sa kin wi- 
cayutecapi kta on waśte.

W. Hena tuwepi e Ağuyapi qa Miniśa kin yutapi qa yatkanpi kta iyececa he?

A. Wakantajka taoyatepi kin owasin.

W. He Tuwe e heconwicaśi he?

A. Jesus Christ Itancan kin.

Tohinni wicaxa wan rtayetu-wotapi tanka wanji kaga, unkan wicaxa nina ota rcin wicakico. Wotapi kta iyehantu kin-

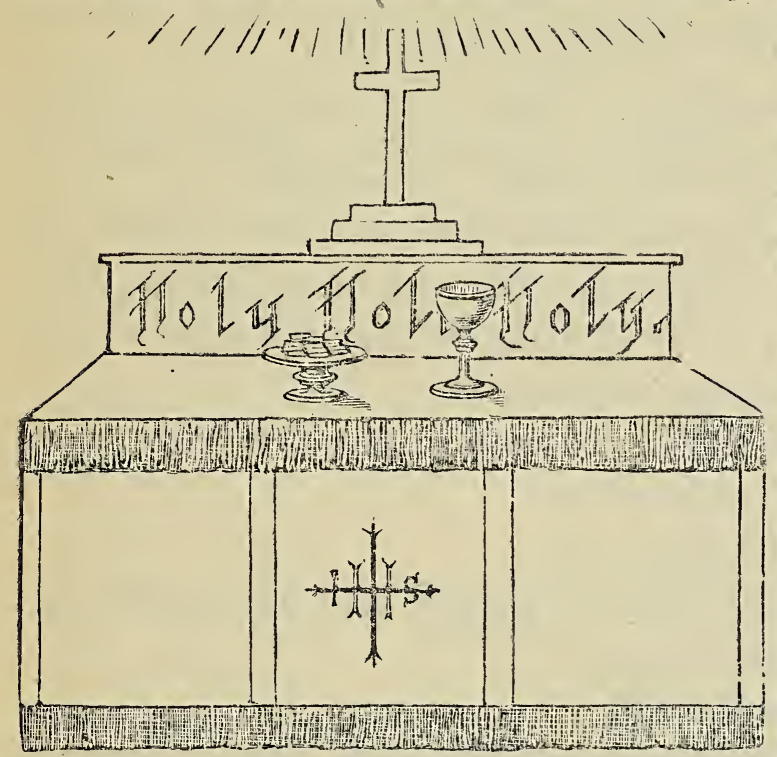
han, makoce kin he ed wicoran kin okna, onaronwicayin kta on tawowaxi wanji ye. xi; tka wicakico qon iyohina ikda tokan ayapi. Wanji wica $\times a$ jica, unkan wotapi kta ed ye xni tka ee maga qa tipi decana op eton hena wankdag yin kta cin. Tokeca wanji wowaxi ofa econ kta iyececa keya, unkan he ayuxtan kta okihi xni kecin. 'Tokeca wan. ji tiyata woawan. yake qa woiyokipi nina ota yuhe cin on kicunipi kta hee exa icekiye xni, tka yin kta okihi xni ce wancakna eya ayupta. Heced econpi kin he nina wawapidaye $x u i$, icin wicakico kin he pidawicayin kta iyececa oran dakax, unkan toked ayuptapi naron qehan ixikda qon he hecetu. Hehan otonwe canku oknakna warpanicapi kin hena owasin wotapi kte cin ekta wicakico kta on tawowaxi yexi, unkan iyuxkinyan hipi. Exa nahanrcin wakna-wotapi ed okan canke ake yexi, otonwe qed okxantanhan icimani hipi, qa togiapi kin hena koya wicakico kta eheon. Hena nakun hipi, unkan wicaxa waxte qon wicayawaxte qa takuku wicaqu kin on, qa tokata enpetu iwicakicihan ed tipi tawa kin okna iye kici tipi kta ewicakiya on nina cantewicayuwaxte. Woyakapi kin de on toked kapi he? Tuwe Wotapi tanka kage ciqon He Wakantanka Ee, unkan he ekta wicaxa owasin wicakico. Hunr ikdatokanyan ayuptapi, qa ake hunr yapi kte xni okdakapi, tka tona yapi kin, wawica- 
qupi nina teririka Wakantanka wicaqu kta, unkan Iye cinca waanagoptanyan un heca ikduhapi hantanhanx, Iye kici Marpiyata ounyanpi wicaqu kta iwahowicaya. Tohan Wicayusutapi ecaniconpi, unkan he ecivatanhan on Christ taakicita qa taokiye henica kta e oyakdake cinhan, wicakicopi kin de nix eya iyoniwaja kta. Hehan Wotapi kin ed de ca Wakantanka woyawaxte tawa kin iyacu oyakihi kta. Ho, he ehand ed nin kta iyececin kta, icin Itancan unkitawapi Rtayetu-wotapi tawa icu unxipi dakax.

\section{$\longrightarrow \mathrm{O}-$ \\ WOONSPE XXXVIII.}

WOONSPE WICOIE-WICONI.

Oekde.- "Tuwe Micerpi etanhan yute ca Mawe yatke cinhan owihanke wanin wiconi yuha." John $6 ; 54$.

W. Mahedtayhay taku eekiyapi kin he taku he?

A. Christ Taycay qa We kin, hena Itaycay Rtayetu-wotapi tawa kin ed, tona wacinyaypi kin, woniya eciyatayhay eyakupi kin hena ee.

W. Itancan Rtayetu-wotapi tawa kin ed Aǵuyapi kin, he taku eekiyapi he?

A. Christ Taycan kin.

W. Itancay Rtayetu-wotapi tawa kin ed Miniśa kii), he taku eekiyapi he?

A. Christ We kin.

W. Christ Tancan qa We kin hena wowakta qaiś wowaśte unma tukte ee he?

A. Wowaśte kin ee.

W. Christ Taycay qa We kin hena toked woniya eciyatanhay i «upi he?

A. Unnag̣ipi kin on unkicupi.

W. Tona Aǵnyapi qa Miniśa kin napcapi kin hena owasin hcin Christ Tancan qa We kin koya icupi he?

A. Hiya, tona wacinyappi kin henana hena icupi.

W. "Tona wacinyanpi kin" ehe cin, he on toked yaka he? 
A. Tona awicakehay Wakantanka iyokipiyapi kta kuwapi kin hena.

W. Tona Christ Tancan qa We kin icupi kin, hena taku yuhapi kta he?

A. Wiconi owihayke wanica.

Moses ciqana, psa ojupi ekna anarmapi qon, he wicaxa tanka icage con hand, Wakantanka he taoyute kin, Egypt makoce tukted taku teririka ota akip a $p$ is a qon hetauhan makoce owanyag waxte, qa taku waxtexte owasin ojun a wan ekta awivaya $x i$.

Nina otapi, unkan tehan omani yapi kta. Canku okna yapi kte cin he atay a kinin makoskantu tukted woyute wanica okna rpaya, exa takuna icakijapi xni.

Anpetu iyohi hihanna eca taku wan cikciqana, kmi k be n a qa rewanke iye-

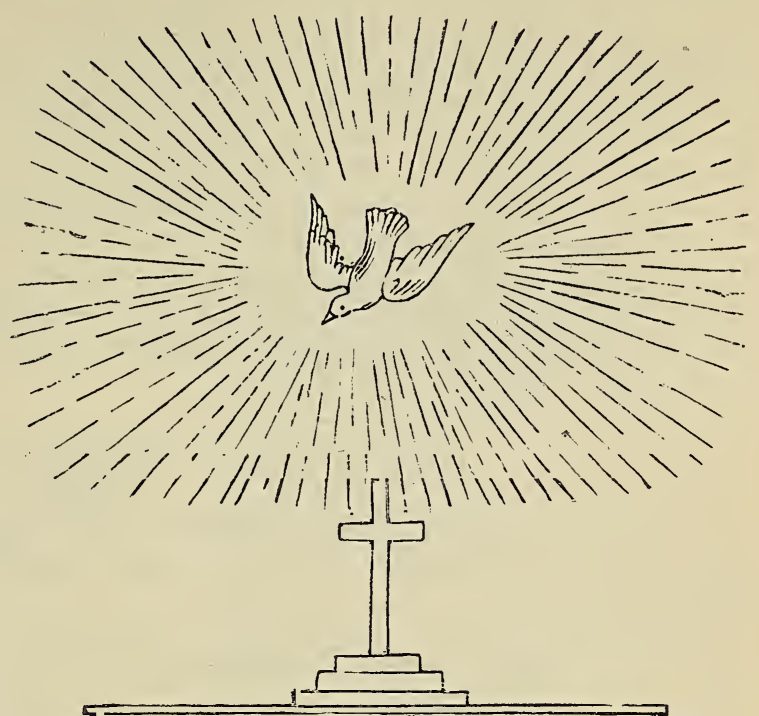
ceca, maka akard iyeyapi kta Wakantanka ewicakiya. Unkan Wakantanka ewicakiye cin iyeced iyeyapi. Aguyapi tu rmaga-canhanpi kici yutapi kin he iyeced skuya, unkan tohanyan cinpi kin iyenakeca mnayanpi okihipi. Anpetu-okirpapi kin hehan ecena takuna iyeyapi $x n i$, icin Wakantanka Anpetu-wakan tawa kin icunhan okirpapi kta cin, qa heon itokab anpetu kin ohinni nonpa akikde wicagu ece. Oyate 
kin woyute kin de "manna" eya cajeyatapi, "Taku huwo?" he heced kapi, icin he taku hecinhan sdodyapi xni dakax. Nakun "oknikde-wakan tawote" eciyapi, Wakantanka he uya heon etanhan, unkan winiyetu wikcemna topa he on makoskantu wodwicaya. Wakantanka heced taoyate ni wieayuha.

Wakantanka untancanpl wowicaqu kin he iyeced nakun unnagipi wowjcaqu. Untancanpi kin aguyapi qa nakun woyute toktokeca yuhapi kta iyecece cin hena wicaqu-taku owasin Iye etanhan unkicupi. Unnagipi kin on woyute wan unqupi kin he Christ Tancan qa We kin hena ee. Wakantan. ka Noah Ark ed un qehand nakun woqu, qa miniunkakaxtanpi qon eciyatanhan on Ark tawa, Okodikiciye Wakan kin hee, timahed unhiyupi kin unkix eya woyute wanji iyotan unnagipi iwaxtepi kta iyecece cin he on wodunyanpi. Tona woyute kin de yutapi kin "tohinni docin unpi kte xni," qa tona Christ We kin yatkanpi kin "tohinni ipuza unpi kte xni," tka wiconi owihanke wanin yuhapi kta, he Christ opewicalsicaton, Tancan qa We tawa roe cin Canicipawega akand otkeyapi kta e icicu qon he eciyatanhan on.

\section{WOONSPE XXXIX.}

WOONSPE WICOIE-NAGI. $121 ; 7$.

Oekde.-"Itancan kin Ninagi kin awanyakin kta." Ps.

W. Heon wowaśte unhapi kin hena tukte e he?

A. Untancanpi kin Ag̀uyapi qa Miniśa kin on iwaśake cin, he iyeced unnaǵipi kin Christ Tancal qa We kin on iwaunśakapi kta.

W. Ag guyapi qa Mijiśa hena toked untancanpi kin iwaśte he?

A. Hena tancal zani qa waśaka unkag̉api.

W. Nakur toked zani qa waśaka ußkorpi kta iyececa he?

A. Naği eciyatanhan.

W. Nag̀i eciyatanhan zani qa waśaka unkağapi kte cin he tukte e he?

A. Christ Tancan qa We kin.

W. He tokeca e untancanpi kin hecena śni tka unnağipi kin nakuı eciyatanhay zaniyan uịkonpi kta iyececa he? 
A. Unnaǵipi kin Malipiyata Wankantanka kici wiyuśkinyal unpi kta $\theta$ heon.

W. Wicanagsi țapi kin he taku he?

A. Wakantanka atokayyal oulyyewicakiyapi kin heo.

W. Malipiyatanhan woyute kin de unkicupi ehantanhayś unnag̊ipi kin ed ounyin kte cin He Tuwe he?

A. Woniya Wakal kin Hee.

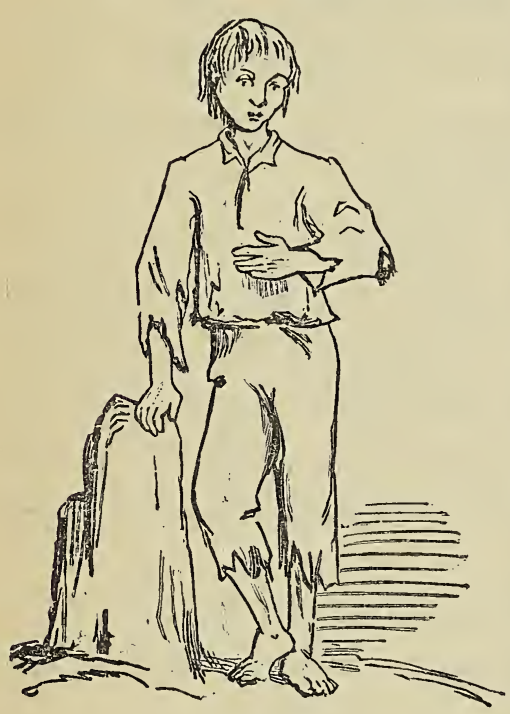

Hokxina wan ite cceca sni ska rea, qa hayapi r'er'e kin de tuwe he? He awicakehan onxidapi kta iyececa hececa, icin tonpi ehantanhan maka-tipi okpaza wan okna ti. Tohinni tanyan wote xni, qa hayapi rderdecahan tawa kin tancan ataya akarpin kta iyohi xni. Tohinni oniya taku xica icahiye xni qa teca se, niya xni, qaix makobdaye toya wanke cin tohinni ekta omani xni. Tha wanna wicaxa waxte qa waonxida wan wojupı makobaxpe wan ekta ayin kte, ca hed waonspekiyapi kta. Tokaheya, tanyan yujajapi kte ca paha kicicakcapi unkan yuksapi kta. Hehan hayapi rdecahe cin dena eekiya hayapi waxte qupi kta, qa canhanpa qa hunyakon waxtexte koya. Wojupi mahobaxpe kin ekta tipi waxte oti kta, unkan tapon xaxa qa cepa icagin kte, ca ixta ececa xni mahed ivaye cin hena ake eced han qa wiyakpakpin kta. Wowapi onspeiciciye, ca wowaxi econ, qa xkatin kta onspe ayin kta, niye ecanon kin iyececa. Hokxina kin de he yutokecapi wan tanka qa waxte heca kta, ci?

Tka wakanheja akantanhan wanwicayakapi kin eciyatanhan tapon xaxapi qa cepapi seececapi, exa tukted onxpa akiran tapi qa tamahedhecapi, hokxina wan ded iteowapi kin he iyececa, heca tuktekted wanwicunyakapi ece. Eya, nagipi kin hena waka. Wicanagi heca wicatancan nina zani rca okna ounyanpi, exa hena Baptisma wicaqu up qa heced he eciyatanhan on skaya wicayujajapi xni ekantanhanx, qa Wakantanka sdodyapi qa teridapi kta onspewicakiyapi xni, qa Itancan Rtayetu- 
wotapi tawa kin on wodwicayapi xni hecinhan, tohinni waxaka qa zaniyan unpi okihipi kte xni.

Wakanheja onxika hececa ota Wakantanka on onspewicakiyapi ikta en Wotanin Waxte ayapi tka womnaye econkonpi qa yeunyanpi ece, unkan nakun makoce kin dedtu exa nina otapi. Hena owicayakiyin kta yacin he? Hena owasin Iye cinca wacinyepica heca i sagapi kte, ca Iye nagi tawapi kin awanwicayakin kta e Wakantanka iceyakiyin kta oyakihi.

\section{WUONSPE XL.}

WOONSPE WICOIE-OKIDE.

Oekde.-CCanku unkokidepi qa unkakitapi kta." Wicaceya, $3 ; 49$.

W. Tona Itaycan Rtayetu-wotapi kın ed upi kte cin taku econpi kta iyececa he?

A. Taninyan woahtani yuhapi kin on awicake- . haf iyopeiciyapi hecinhal) okidepi, qa sdodkiyapi kta, awicakehay teca ni ed togye olianyarjpi kta içiconzapi kta; Christ eciyatanhay Wakantanka towaonśida kin awicakehan wicadapi, qa Iye to cin he pidaya kiksuyapi kta; qa wicaśa owasin ob całtekiciya uypi kte cin hena ee.

W. "Okide" eyapi kin he on toked kapi he?

A. Taku qeyaś itompeya akicikcitapi kiniwayiçikdakapi kin.

W. He taku e sdodumkiyapi kta e on itonpeya unkakicikcitapi qa iwayunkiçikdakapi kta he?

A. A wicakehan waunhtanipi kin on icanteunsicapi hecinhay, hee.

W. Nakuy taku on uncantepi kin ed unkokidepi kta iyececa he?

A. Wakantanka wacinunyaypi hecinhay sdodunkiyapi kta e he ol).

W. Qa toked canteyus unkoypi kta iyececa he?

A. Pida uykoypi kta. 
W. Nakul taku ecolkoljpi kta iyecera he?

A. Wicaśa owasin ob cantekiciya un kol i k ta.

W. Akicikcitapi qa iwaljiçikdakapi lita iyececa kin, heya tola eepi he?

A. Tona Itancal Rtayetu-wotapi tawa kin ed yapi kte cin.

W. Niye kiıhaı, ni(-ałte iwaıniçikdakiı kta iyececa he?

A. Ho, hayhepi iyohina.

W. 'Tokeca e hecanoly lita iyececa he?

A. W akantanka wawalitaıi kin micicajuju kta e, qa Iye tewahida kta e omakiyin kta, hena on etaıhay.

Wicaxayatapi ti kin ekta wotapi wan kagapi kta unkan he ekta wakanheja ota wicakicopi. Taku wauji tiusyaked eci:awicaxipi-iyohina cuwiknaka ska rea, tuktedua sapsapena owapi xni, qaix woaxape exa wanica, heca kicun hipi kta hee. Ht ceca ece kicun tanyan hipi kta eheon Wicaxa. yatapi kin nakun hewicakiya, miyokdasin okar tokeca tipi iyohi ed otkeya he cin hena ed tanye rein cliwiknaka iwankdakapi kte, ca hehan miniyowe $x$ an Wicaxayatapi wojnpi tanka tawa tiyopa kin ikiyena kaduze cin he ed woaxape kin owasin tanyan yuskapi kta. Wakauhejapi kin hunr Wicaxayatapi toked econwicaxi qon he itonpeya eced econpi. Qa hunr, cuwiknaka nina ed ekitonwanpi xni wancakna miniyowe $q^{\wedge n}$ ekta ahi minin oknakapi. T'ka wicincana wanji, toked oyate ekna heca ayucoya kagapi ece in he iyc cera cuwiknaka wanji icicaga, unkan be

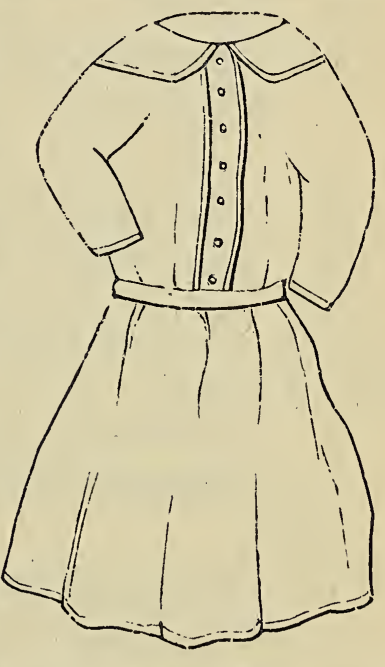
nina owanyagwaxte qa wotapi kin ekta kicun yin kta iyecrd ska rea kecin. Canke be ed woaxape yukan hecinban ecara ode xni. qa miniyowe ekta yin kta takun awacin xni. $\mathrm{K}$ ana wotapi ekta yapi kta anpetu kin ohihanna kin he kicun, $n 11$ kan heehand woaxape wanji ix noupa wanyaka, exa hena inarni makasan on askakiya unkan wakanheja unmapi kin ob Wicaxayatapi ti kin ekta iyaya. Wicaxayatapi kin ly e rea qa wowaxi wicayube cin hena owasin hasapi wiyak pa ska lia kicunpi, qa wakanheja tona Wicaxayatapi toahope anagoptan- 
pi kin ix eya cuwiknaka wiyakpe xni qeyax owasin woaxape wanin skaska rca ececapi.

Tka hehehe! Wanji cuwiknaka kdujaja kta akta xri qon he toker wowixtece tanka akipa ced! Cuwiknaka tawa kin woaxape sapsapa on nina xapa, qa onxpaxpa makacan on skakiye ciqon hena ix ilotan owanyagxikxica rea. Xina apardate on ayucoya kagapi ciqon hena wana niniruha ordecahan hectca seececa otanin. Tohan Wicaxayatapi wanyaka unkan he tokeca e cuwiknaka ska kicun hi xnj he? eya iwange cehand, wicoie wanjina exa on ayuptin kta okihi xni, tka wakanheja cantewaxtepi kin qa rcin ira awicatonwan najin kin hena efanhan cancanyan qa wowixtece yuha wowaxi unpi kin on itehan yusayapi.

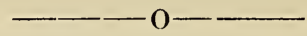

\section{WOONSPE XLI.}

AKTA ONSPEWICAKIYAPI.

W. Christ Wakicolzapi tonakeca econwicas̉i he?

A. Norjpa.

W. Hena taku eciyapi he?

A. Baptisma qa Italycal Rtayetu-wotapi tawa $k i n$.

W. He tuwe e hena ecorwicasi he?

A. Jesus Christ Itancan ußkitawapi kin.

W. Wakiconzapi wayji ed oyśpa nonpa kiı hena tona ee he?

A. Wowakta kin qa wowaśte kiı.

W. Baptisma ed wowakta kin he tukte e he? Mini.

W. Baptisma ed wowaśte kin he tukte e he?

A. Woahtani wicakicicajujupi qa Wakantanka Woniya Wakan tawa kin.

W. Baptisma wicaqupi kte cin hena itokab taku yuhapi kta he?

A. Woiyopeiçiye qa wowacinye:

W. Heced tokeca e hokśiyopa baptisma wicaqupi he? 
A. Itancal ulkitawapi kin heya, "Hokssiyopa ed maupi kta iyowinwi(akiya po," he ol etanhay.

W. W'oiçicolyze ewicakiciyapi kte ca iyopeiciyapi kte ça wicadapi kta olsspewicakiyapi kte cin hena tuwepi he?

A. W aewicakiciyapi kil).

W. Itajcan Rtayetu-wotapi tawa kin econkonpi calna kiksu! a uıkoıpi kta iyecere cin he taku he?

A. Christ canicipaweğa akand unkiyepi on te ciqon hee.

W. Itancan Rtayetu-wotapi tawa kin ed wowakta kin he tukte e he?

A. Ağuyapi qa Miniśa.

W. W owastte kin he tukte e he?

A. Christ Tajcal ya We kin.

W. He eciyatanhan ol wowasite unkicupi k te cil he taku he?

A. Uynaǵipi kỉ zani qa waśakapi kta.

W. Tona Itancan Rtayetu-wotapi tawa kin ed yapi kte cin taku econpi kta he?

A. Walitanipi kin ol iyopeiçiyapi, qa wicadapi, qa wicaśa ul)mapi kiı cantewicakiyapi hecinha! iwaniçikdakapi kta.

W. He taku e Christian nicağin kta he?

A. Baptisma.

W. He taku e Christian wacinyepica yaun kta e okihiniyin k ta he?

A. Itancan Rtayetu-wotapi tawa ki1).

W. Baptisma on iteowapi yeksuyin kta oyakihi kin tona ee he?

W. Itancan Rtayetu-wotapi tawa kin on iteowapi yeksuyin kta oyakihi kin tona ee he? 



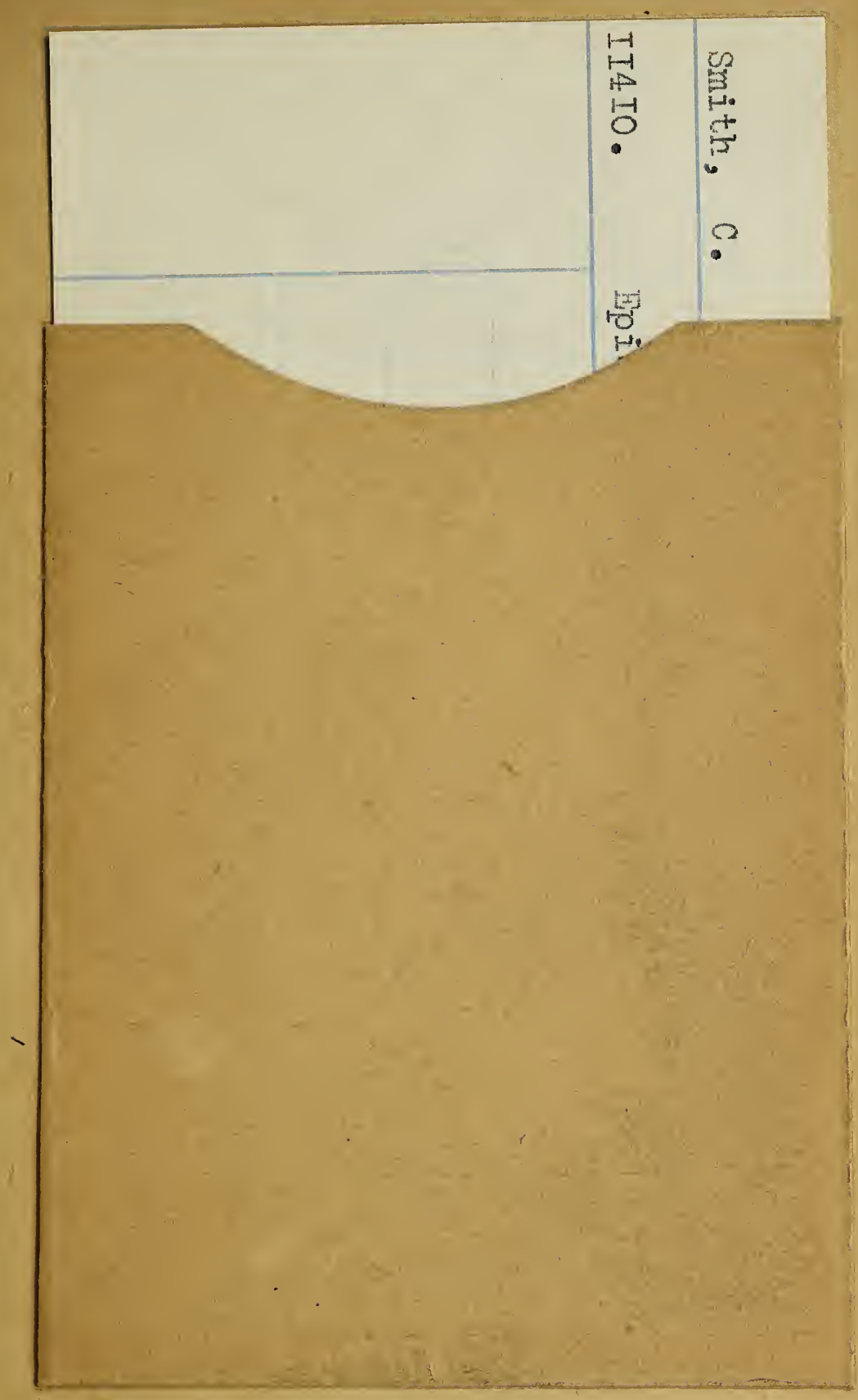


SMITHSONIAN INSTITUTION LIBRARIES 